2014

\title{
INVESTIGATING THE ROLE OF THE CONTACT LINE IN HETEROGENEOUS NUCLEATION WITH HIGH SPEED IMAGING
}

Colin Gurganus

Michigan Technological University

Follow this and additional works at: https://digitalcommons.mtu.edu/etds

Part of the Atmospheric Sciences Commons

Copyright 2014 Colin Gurganus

\section{Recommended Citation}

Gurganus, Colin, "INVESTIGATING THE ROLE OF THE CONTACT LINE IN HETEROGENEOUS NUCLEATION WITH HIGH SPEED IMAGING", Dissertation, Michigan Technological University, 2014.

https://doi.org/10.37099/mtu.dc.etds/888

Follow this and additional works at: https://digitalcommons.mtu.edu/etds

Part of the Atmospheric Sciences Commons 
INVESTIGATING THE ROLE OF THE CONTACT LINE IN HETEROGENEOUS NUCLEATION WITH HIGH SPEED IMAGING

\author{
By \\ Colin Gurganus
}

\begin{abstract}
A DISSERTATION
Submitted in partial fulfillment of the requirements for the degree of DOCTOR OF PHILOSOPHY

In Atmospheric Sciences
\end{abstract}

MICHIGAN TECHNOLOGICAL UNIVERSITY

2014

(C) 2014 Colin Gurganus 

This dissertation has been approved in partial fulfillment of the requirements for the Degree of DOCTOR OF PHILOSOPHY in Atmospheric Sciences.

Department of Physics

Dissertation Co-Advisor: Dr. Raymond Shaw

Dissertation Co-Advisor: Dr. Alex Kostinski

Committee Member: Dr. Paul Bergstrom

Committee Member: Dr. Will Cantrell

Department Chair: Dr. Ravi Pandey 



\section{Contents}

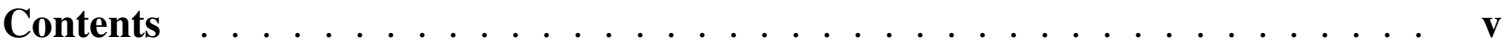

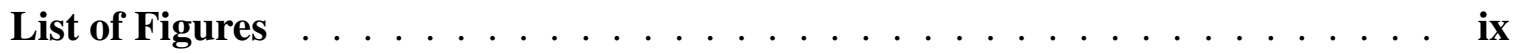

Acknowledgments $\ldots \ldots \ldots \ldots \ldots \ldots \ldots \ldots \ldots \ldots \ldots \ldots \ldots \ldots$

Preface $\ldots \ldots \ldots \ldots \ldots \ldots \ldots \ldots \ldots \ldots \ldots \ldots \ldots \ldots \ldots \ldots \ldots$

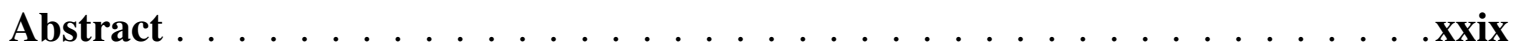

1 Background ....................... 1

1.1 Atmospheric Nucleation $\ldots \ldots \ldots \ldots \ldots \ldots$

1.2 Nucleation Theory . . . . . . . . . . . . . . . . . . 2

1.2.1 Nucleation Rate . . . . . . . . . . . . . . . . . . . . 3

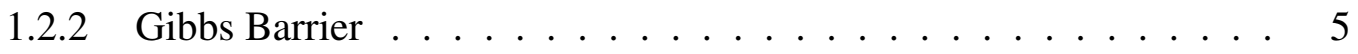

1.2.2.1 Homogenous Nucleation . . . . . . . . . . . . 5

1.2.2.2 Heterogeneous Nucleation . . . . . . . . . . . 7

1.2.3 Line Tension . . . . . . . . . . . . . . . . . . . 10

1.3 Homogeneous Surface Crystallization . . . . . . . . . . . . . 12

1.4 Heterogeneous Surface Crystallization (Contact Nucleation) $\ldots \ldots \ldots$

1.4.1 Nucleation by External Crystal "Cooper mode" . . . . . . . . . . 14

1.4.2 Thermal Gradients . . . . . . . . . . . . . . 15 
1.4.3 Line Tension . . . . . . . . . . . . . . . . . . . . . 17

1.4.4 Surface Texture . . . . . . . . . . . . . . . . . . . . . . 27

2 Experimental Methods $\ldots \ldots \ldots \ldots \ldots \ldots \ldots$

2.1 Research Outline . . . . . . . . . . . . . . . . . . . . . 31

2.2 Characterizing Droplet Nucleation $\ldots \ldots \ldots \ldots$. . . . . . . 32

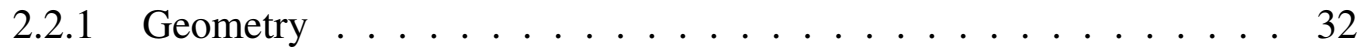

2.2.2 Freezing Characteristics of Supercooled Droplets . . . . . . . . 36

2.2 .3 Imaging $\ldots \ldots \ldots \ldots \ldots \ldots \ldots \ldots$

2.3 Isothermal Chamber $($ Chapter 3$) \ldots \ldots \ldots \ldots$

2.4 Thermal Chamber $($ Chapter $4-5) \ldots \ldots \ldots \ldots$

2.5 Probing the droplet (Chapter 5) . . . . . . . . . . . . . . . 41

2.5.1 Poking Apparatus . . . . . . . . . . . . . . . . . 41

2.5.2 Nano-textured Optical Fibers . . . . . . . . . . . . . . . . . . 42

3 The Role of a Three Phase Interface $\ldots \ldots \ldots \ldots$

3.1 Abstract . . . . . . . . . . . . . . . 46

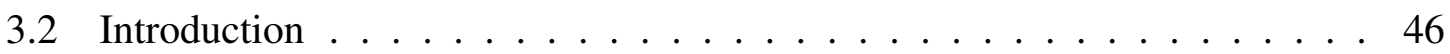

3.3 Experimental Methods . . . . . . . . . . . . . . . . 57

3.4 Acknowledgement . . . . . . . . . . . . . . . . . . . . . . . . . . 59

4 The Role of Thermal Gradients . . . . . . . . . . . . . . . . . . . . . . 61

4.1 Abstract ......................... 62

4.2 Introduction . . . . . . . . . . . . . . . . . 63

4.3 Experimental Techniques . . . . . . . . . . . . . . 65

4.4 Results . . . . . . . . . . . . . . . . . . . . . . . 67

4.5 Discussion . . . . . . . . . . . . . . . . . . . . . . 69 
4.6 Acknowledgement . . . . . . . . . . . . . . . . . 74

5 The Role of Surface Texture . . . . . . . . . . . . . . . . . . . . 75

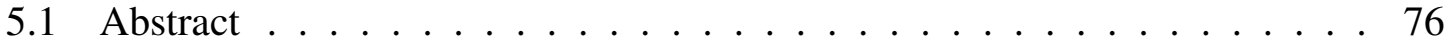

5.2 Introduction $\ldots \ldots \ldots \ldots \ldots \ldots$

5.3 Acknowledgement . . . . . . . . . . . . . . 87

5.4 Methods .............................. 87

5.4.1 Thermal Control Method . . . . . . . . . . . . . . . 87

5.4.2 Spatial Identification of the Nucleation Site and Results for Micro-Texture . . . . . . . . . . . . . . . . . . 89

5.4.3 Fabrication of Micro-Texture Features . . . . . . . . . . . . . 91

5.4.4 Fabrication of Nano-Texture Features . . . . . . . . . . . . 92

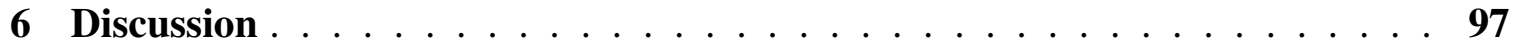

6.1 Spatial Distributions of Nucleation Sites on Substrates . . . . . . . . . . . . 97

6.2 Freezing Temperature Distributions . . . . . . . . . . . . . 101

6.3 The Importance of Surface Features _ . . . . . . . . . . . . . . . 105

6.4 Conclusion . . . . . . . . . . . . . . . . 108

6.5 Other observations and Future Work . . . . . . . . . . . . . . . . 109

6.5.1 Point-Like Contact . . . . . . . . . . . . . . . . . 109

6.5 .2 Capillary Waves . . . . . . . . . . . . . . . . 111

6.5.3 Bubble Nucleation ................... 111

6.5.4 The Role of Humidity . . . . . . . . . . . . . . . . . . . . . 116

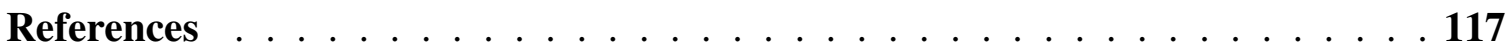





\section{List of Figures}

1.1 Nucleation of Modes in Tropospheric Clouds Homogeneous and heterogeneous nucleation of cloud droplets can occur at different elevations in tropospheric clouds. A rising air parcel will be cooled at the atmospheric lapse rate $\Gamma<10 \mathrm{~K} / \mathrm{km}$, raising the droplets into the heterogeneous and homogeneous regimes respectively $\left(T_{\text {Melt }}>T_{\text {Heterogeneous }}>T_{\text {Homogeneous }}\right)$. Above the heterogeneous freezing threshold, supercooled water droplets and ice particles can exist simultaneously in a "mixed-phase" cloud. The largest ice particles, not necessarily formed by homogenous nucleation, grow via the Bergeron process or conglomeration until they become to heavy to be transported by internal cloud eddys and fall out of the cloud

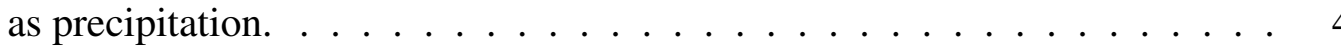

1.2 Formation of Nucleus on External Catalyst: A schematic of ice formation on an catalyst particle. Before crystallization of the entire droplet can proceed, a spherical cap nucleus $\left(r_{\text {nucleus }}>r_{\text {critical }}\right)$ must be formed. Crystallization will then spread out from this location until the entire droplet is frozen. In this situation the nucleus forms at the two phase-interface, and would be considered immersion mode nucleation. . . . 8 
1.3 Examining the shape of the energy barrier to nucleation $\Delta \mathrm{G}$ (equation 1.14) when line tension (C) is included. Typical values for $\mathrm{A}$ and $\mathrm{B}$ derived from equation 1.9 with $l_{f} \sim 10^{8} \mathrm{~J} / \mathrm{m}^{3}$ and $\sigma_{\text {ice-water }} \sim 10^{-1}$. The three lower lines (green, pink orange) represent increasing values of line tension. For line tension values greater than $|\tau|=10^{-9}$ the barrier to nucleation no longer exists, which suggests that this is a fundamental limit to the magnitude of $\tau \ldots \ldots \ldots \ldots \ldots \ldots \ldots$

1.4 Examples of the various scenarios for droplet-particle (triangles) interactions. A. Fully immersed particle B. Partially immersed particle C. Point-Like contact of particle at droplet interface D. Point-Like contact "Inside-Out" E. A particle with an ice crystal on the surface coming into contact with the droplet $\mathbf{F}$. A particle coming into contact with a droplet that is coldest at the interface due to rapid cooling. . . . . . . . . . . . . . . 14

1.5 Homogeneous Nucleation: Volume vs. Surface To illustrate the difference between surface and volume nucleation, we consider homogeneous nucleation of a cubic critical nucleus with side length $\delta$. Each face of the crystal has a corresponding surface tension. For volume freezing all faces of the nucleus are in contact with water $\left(\sigma_{\text {Ice-Water }}\right)$, but in the surface configuration one of the faces is exposed to the air $\left(\sigma_{I c e-A i r}\right)$. Additionally, the four edges surrounding the Ice-Air interface are in contact with all three phases (Ice, Air and Water) and will have a line tension energy $(\tau)$ 
1.6 Heterogeneous Nucleation: Immersion vs. Surface To illustrate the difference between surface and immersion nucleation, we consider heterogeneous nucleation of a cubic critical nucleus with side length $\delta$. Each face of the crystal has a corresponding surface tension $\left(\sigma_{\text {face }}\right)$. This geometry is identical to that in Figure 1.5 except one face of either crystal is in contact with a catalyst substrate. . . . . . . . . . . . . 22

1.7 A scanning electron microscope (SEM) image of a mineral dust particle. Note the fine scale surface texture and sharp features. Many small mineral dust particles are transported into the troposphere, where they can interact with supercooled cloud water droplets. . . . . . . . . . . . . . . . 24

1.8 Macroscopic vs. Microscopic Contact Line, Left: A spherical droplet rests on a catalyst substrate in a similar geometry to that of Gurganus et al.[31, 32] Right: An enhancement of the region in the vicinity of the droplet contact line, highlights the small surface features (black squares) on the "rough" catalyst. When these features are present at the air-water interface, the macroscopic droplet triple line $\left(r_{\tau \text { Macro }}=r_{\text {Drop }}\right)$ is distorted. A new "microscopic triple line" is created with a radius of curvature based on the size of the feature $\left(r_{\tau \text { Micro }} \approx r_{\text {SurfaceFeature }}\right)$. The new "microscopic" triple line may be sufficiently small $\left(r_{\text {TripleLine }} \sim \delta\right)$ that line tension tension contribution $\left(\sigma_{\tau}=\frac{\tau}{r_{\text {TripleLine }}}\right)$ is may increase the probability of surface freezing $\left(J_{\text {Rough }}>J_{\text {Smooth }}\right) \ldots \ldots \ldots \ldots \ldots \ldots$

\subsection{A possible mechanism for point-like contact freezing A sharp aerosol} extruding from the droplet (Figure 1.4 D), may form a spherical cap of supercooled water at the point of contact. Under certain conditions, the line tension contribution may be significant $r^{*}>r_{\text {poking }} \sim \delta$ (Equation 1.27) leading to a contact mode enhancement. . . . . . . . . . . . . . 28 
2.1 Contact Line Similarity The contact line formed at the spherical-cap droplet perimeter is analogous to that one formed at the three-phase (solid-liquid-vapor) interface between an aerosol in contact with a droplet. . 34

\subsection{Contact vs. Immersion Nucleation of a Spherical Cap Droplet A} schematic top and side view of initial crystallization of the drop (dark grey). Contact mode nucleation will begin at the droplet three-phase (substrate-water-air) perimeter line. Immersion mode nucelation will begin in the two-phase substrate-water interracial area. A third scenario where a crystal growing external to the droplet triggers nucleation, in a similar fashion to that described by Cooper [13] is occasionally observed for some substrates, but these instances are filtered from that data and will not be considered here. . . . . . . . . . . . . . . . 35

2.3 Latent Heat Release Warming of the air in the vicinity of a freezing droplet due to latent heat release. The size of the heat spike (subtracting the ambient temperature) is proportional to the volume of the droplet, with the decrease here indicating an evaporating droplet over multiple freeze-that cycles. These air temperature profiles were recorded during the experiments described in Chapter $3[31] \ldots \ldots$. . . . . . . . . . 37

2.4 Identifying the Nucleation Site The nucleation site can be clearly identified by reviewing the high speed nucleation video through the window on top of the thermal chamber. Here, every 5 th frame of a $5 \mathrm{kHz}$ video is shown for a contact mode nucleation event beginning at the three phase interface on the droplet perimeter (left), and an immersion event beginning on the substrate in the two phase region (right). A cartoon for each scenario depicts the spread of the crystal front. . . . . . . . . . . . . . 39 


\subsection{A half cut schematic view of the 2011 Isothermal Chamber A water}

droplet (blue) resting on a silicon wafer (black) sits on a small stand in the middle of the copper lined chamber volume. The chamber body is machined in 4 pieces from Acetal polymer (grey), with channels milled for antifreeze to flow around the copper walls. A viewing window (not shown) located directly above droplet, allows for imaging of the droplet-substrate interface. A plastic measurement package is suspended above the droplet to monitor the volume temperature, and provide off axis illumination. . . . . 40

\subsection{A half cut schematic view of the 2013 Isothermal Chamber A droplet} (white) is cooled by controlling the temperature of the substrate (black) through the use of a solid state peltier cooler. The copper jacketed walls and the heat sink below the droplet are cooled by circulation of anti-freeze as in the previous chamber. . . . . . . . . . . . . . . . 42

2.7 The poker fiber is attached to a plastic rod and inserted into the chamber through one of the port holes in horizontal window ports in the thermal chamber. Positioning of the fiber in the droplet is accomplished with a three axis mechanical stage for course adjustments and a piezo electric linear stage for fine adjustments. A right angle endoscope (green) was used for alignment is some situations. . . . . . . . . . . . . . . . 43 


\subsection{Nano-Scale Surface Features on an Silica Fiber A cascade of SEM}

images for a fiber that has had its plastic cladding removed by etching in a sulfuric acid bath for $\approx$ one hour. At the lowest image resolution (top left), the fiber diameter $(\mathrm{d} \approx 145 \mu \mathrm{m})$ appears to be smooth with a few dust particles deposited on the surface. Zooming in on one of the dust particles (top right) reveals an array of smaller scale surface features. At the highest resolutions (bottom) the features appear circular and have a distribution of sizes down to 10-20nm. . . . . . . . . . . . . . . . . . 44

3.1 Experimental design and nucleation geometry. (Left) Schematic side view of the experimental setup. The high-speed camera is configured to pinpoint locations of freezing events on the substrate. The drop rests on a clean homogeneous and isothermal silicon wafer. The droplet and substrate are encased in an isothermal box, and the walls cool slowly, thereby minimizing temperature gradients. (Right) Schematic perspective view of two freezing drops, illustrating an interior substrate event (immersion mode) and an event occurring at the triple line (contact mode) . . . . . . . 50 
3.2 Deviation from thermal homogeneity (equilibrium) within drops, subjected to steady cooling. Here, we present likely thermal variations $\Delta \mathrm{T}$ for various experiments, estimated on the basis of thermal relaxation time $\tau$ and applied cooling rate $\lambda$. The thermal relaxation time is estimated using a characteristic length scale $V^{1 / 3}$ (for aspherical drops). Contours of the experimental control parameter $\lambda$ are also shown. Points are shown for the conditions of the homogeneous experiments of Bauerecker[3] (1.5 and 4.5 mm acoustically levitated drops placed in a chilled volume, with a cooling rate of $20 \mathrm{~K} / \mathrm{min}$ estimated from their Figure 3.3; droplets had varying salt concentrations) and Hindmarsh[36] (spherical droplet suspended from a thermocouple with a cooling rate of $90 \mathrm{~K} / \mathrm{min}$, as estimated from their Figure 3.2; droplets had varying sucrose concentrations), as well as the heterogeneous experiments (spherical cap droplets on an actively cooled substrate) of Fornea[23], Gurganus (presented here), Shaw [19], and

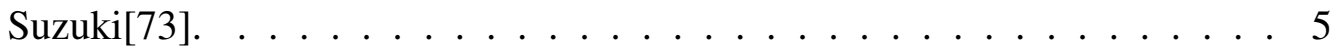

3.3 Progressions (time evolves downward) of contact (left) and immersion (right) nucleation events for a $10 \mu \mathrm{L}$ spherical cap droplet, viewed from above. The images have been enhanced for contrast, with the freezing front appearing in gray and bright LED reflections evident. In the immersion film, the nucleated crystal is oriented with the basal plane aligned with the imaging plane, thereby showing a hexagonal shape, but this is not necessarily the preferred orientation. These videos were recorded at 20,000 frames per second, with every 20th frame displayed (0.001s time steps between images). A movie of the two freezing events (played at $6 * 10^{4}$ times live speed) is available as Supporting Information. . . . . . . . . . . . 53 
3.4 Evidence for spatial uniformity of nucleation events. Contrary to expectations, there is no preference for perimeter (contact mode) nucleation. (Top) Locations of the 189 freezing events on the horizontal plane. Radial positions have been scaled by drop radius to facilitate comparison between runs. (Bottom) An equal area histogram of 10 circular bands provides a quantitative measure of spatial uniformity. The solid horizontal line denotes the expected number of events per bin [4] assuming uniform distribution, and the dashed horizontal lines mark the Poisson

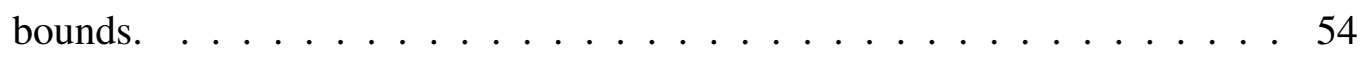

4.1 Schematic of the new thermal chamber. Continuing the geometry of refs 1 and 2, this study examines supercooled droplets in contact with an atomically smooth $\mathrm{Si}$ substrate. Unlike our prior study, the substrate is placed in thermal contact with a Peltier cooling element (white) to achieve high cooling rates $(5 \mathrm{~K} / \mathrm{min})$. The droplet and cooling element are contained in an isothermal chamber with positive pressure from a dry air line. Optical ports on the top and sides of the chamber allow for simultaneous imaging of the droplet in the horizontal and vertical planes. . . 64

4.2 Pair of high-speed Photron SA2 cameras allows us to pinpoint nucleation sites and measure the droplet contact angle. In this film strip each frame represents a time step of $2 \mathrm{msec}$, or every 20th frame at a sample rate of 10 $\mathrm{kHz}$. The nucleation site is evident at the center of the hexagonal crystal when the film is viewed in reverse. . . . . . . . . . . . 66 
4.3 From image analysis of the top-view movies, we can determine the nucleation site at the droplet-substrate interface. In the left panel, nucleation sites for a droplet with low contact angles on a clean silicon wafer are displayed in red. In the right panel, nucleation sites for a droplet with higher contact angles on a silanized wafer are displayed in blue. The dashed lines separate regions of equal area (10\%).[31] Both distributions appear uniformly random in agreement with our previous observations . . . 69

4.4 Image analysis from the side-view camera allows us to determine the contact angle of each droplet at the initiation of freezing. The red points indicate observed nucleation events on a clean $\mathrm{Si}$ wafer, while the blue points indicate observed nucleation sites on a silanized Si wafer. As in Figure 4.3 , the dashed lines separate 10 zones on equal area to help guide the eye in the radial distribution of sites. The large variability in the observed contact angles of both sets is caused by pinning at the triple line during slow evaporation of the droplet. The approximate ranges for the observed drop contact angle, perimeter, and height are respectively $30^{\circ}-90^{\circ}, 10-30 \mathrm{~mm}$, and $1-2.5 \mathrm{~mm}$. There appears to be no difference in the distribution of nucleation sites with contact angle. . . . . . . . . . 70 
4.5 Results of our thermal analysis of this geometry using the Solidworks software package. This simulation mimics our experimental conditions, with the droplet, wafer, and heat sink initially at a stable equilibrium with the isothermal chamber $(275 \mathrm{~K})$. At $\mathrm{t}=0$, the temperature of the heat sink is decreased at a rate of $5 \mathrm{~K} / \mathrm{min}$ for $360 \mathrm{~s}$ until it falls to a substrate temperature of $245 \mathrm{~K}$, at which the temperature of the heat sink is held constant until the droplet comes into equilibrium. The bottom panels represent data from nodes at the top and bottom of the droplet. The first node is on the droplet-silicon interface. The selection of this bottom node is arbitrary because we observe no thermal gradient at the droplet-substrate interface. The second node is located at the apex of the droplet $2.5 \mathrm{~mm}$ above the interface. The left panel displays the raw temperature at both nodes during the cooling cycle. The right panel displays the thermal gradient that develops between these two nodes. After an initial transient stage $(60 \mathrm{~s})$, a steady state gradient of $1.5 \mathrm{~K}$ is maintained throughout the cooling cycle. After the active cooling ends (360 s), the droplet relaxes exponentially toward the new equilibrium temperature. . . . . . . 71

5.1 Fabricating nanoscale surface texture. Motivated by the conjectured importance of roughness to heterogeneous nucleation and the plausible range of length scales, these experiments were conducted with smooth optical fibers (A,B), nano-textured optical fibers (C,D), and micro-textured silicon substrates (see supplemental Figure 5.5) as heterogeneous nucleation catalysts. Panels A-D were taken with a high resolution SEM. $\quad$. 80 
5.2 Three modes of nucleation. Top: A schematic of the droplet-fiber geometry. A $30 \mu \mathrm{L}$ droplet with a contact angle of $\approx 90^{\circ}$ rests on a siliconized glass slide (Hamilton Scientific) that is cooled from below [32]. An optical fiber, partially immersed within the droplet, can act as a heterogeneous nucleation catalyst. Three possibilities for nucleation then arise: on the substrate (red), on the immersed fiber (green) and at the fiber contact lines (blue). Bottom: By imaging the crystallization at $5 \mathrm{kHz}$ we pinpoint the nucleation site (boxed area in film strips). Film strips here represent each of the three nucleation modes. Every 15th frame is shown resulting in a 3 ms spacing. . . . . . . . . . . . . . . 84

\subsection{Nano-Texture observed to cause a transition to surface nucleation at}

the contact line. Results for the three modes of nucleation, substrate, immersed fiber, fiber contact line (see Figure 5.2). Left Panel: Spatial origin of crystallization is observed often to shift to the contact line for nano-textured (rough) fibers, but neither the smooth fiber $(r=70$ $\mu \mathrm{m})$ nor the micro-textured substrates $(2-100 \mu \mathrm{m})$ yield such a shift (see supplemental material, section 2). Despite relatively small surface area of the nano-textured fiber, over half of freezing events are initiated there. Furthermore, despite the overwhelmingly small spatial odds, the majority of the fiber induced events originate at the contact line. Right Panel: Higher freezing temperatures (weaker supercooling) are observed for nucleation events at the fiber contact line as evidenced by the cumulative freezing probabilities (red curve). Broadening of the distribution accompanies fiber contact line events, as expected because of additional variability in the geometry of the nano-textured contact lines. . . 85 
5.4 A comparison of the distribution of nucleation sites on the micro-textured substrates. Here, we identify the nucleation site at the substrate-water interface in the same way previously described (Figure 5.2 in article). Using the procedure outlined previously $[31,32]$ we classify any nucleation site located near the droplet perimeter, or outermost $10 \%$ of substrate-water interface region, as a contact mode freeze (Left Panel). When we sort the observed nucleation sites into Immersion and Contact freezes (Right Panel), it is clear that the number of sites is proportional to the surface area (observed number slightly exceeds $\pm 1 \sigma$ and is well within $\pm 2 \sigma$, where $\sigma^{2}$ is the Poisson variance), which agrees with our previous observations for smooth substrates $[31,32]$ and the stochastic assumption of classical nucleation theory. This result suggests that there is no enhancement for surface crystallization in the presence of micro-texture surface features. . . 90 
5.5 Fabricating surface texture from $100 \mu \mathrm{m}$ to $100 \mathrm{~nm}$. The profiles here indicate a subset of the micro fabricated substrates that we tested in these experiments. We utilize contact lithography with a reactive ion etch to create large $\left(2.5 \mathrm{~cm}^{2}\right)$ arrays of square wave features on homogeneous silicon substrates. We are able to control the period and depth of the structures to produce a uniform surface texture across the droplet-substrate interface. Here we show three dimensional (left) and two dimensional (right) Atomic Force Microscopy (AFM) surface profiles of a subset of our micro fabricated textured substrates. These substrates have square wave features with periods of $4 \mu \mathrm{m}$ (top), $10 \mu \mathrm{m}$ (middle) and $20 \mu \mathrm{m}$ (bottom) with depths of $\approx 600,300$ and $900 \mathrm{~nm}$, respectively. Here we do not show AFM profiles for the largest period $(100 \mu \mathrm{m})$, because the scan range of the AFM is insufficient to observe multiple features. The deepest etches (depth $\approx 900 \mathrm{~nm}$ ) reveal additional texture in the $100 \mathrm{~nm}$ to $1 \mu \mathrm{m}$ range that result from prolonged exposure to the reactive ion plasma. The range of scales is therefore extended down to the $100-n m$ scale. . . . . . . . . . . . . 93 
5.6 Surface morphology for representative smooth and rough fibers. Here the fibers (classified as smooth (left) and rough (middle) by our classification scheme) were imaged with under similar conditions and resolution with an SEM. The distribution of surface feature sizes, obtained with the ImageJ analysis tool, is indicated as number densities per area (right). For all fibers small scale features $(<10 \mathrm{~nm})$ exist but they are much more abundant on the rough fiber. It is possible that many of the smallest features may be anomalous artifacts from image processing, which would further reduce the density of the smallest features. This analysis excludes any features smaller than $2 \mathrm{~nm}$ because we have sputtered a 2-nm-thick coating of platinum to increase the conductivity of the sample, which was necessary for SEM imaging. It is unlikely that features below this size are relevant for ice nucleation, as they are smaller than the size of a typical critical ice nucleus.

6.1 Droplet-Substrate Spatial Distribution Data Freezing sites are distributed uniformly over the droplet-substrate interface for data from Chapter 3 (right) and Chapter 4 (left). Here the data is derived from Figure

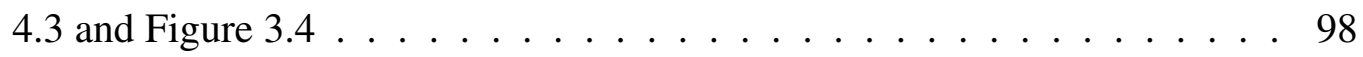

6.2 The effect of glint on the nucleation site detection Here, a hexagonal crystal grows from a nucleation site in the two-phase immersed region (away from the contact line). When viewed from the side it is possible that the projection of the crystal onto the curved droplet surface could be interpreted as freezing at the three phase contact line. . . . . . . . . 100 


\subsection{Freezing temperature $\left(T_{\text {Freeze }}\right)$ distributions from our two substrate}

studies In these studies (Left: Chapter 3, Right: Chapter 4), the temperatures are normally distributed with a clearly defined mean and variance. In our second study, a subset of the silicon wafers treated with RainX exhibit a much lower freezing temperature $(\Delta T \approx 4 K)$ but a very similar variance. This can be partly attributed to the reduction in surface area due to higher contact angle $\left(\Delta \theta_{\text {droplet }} \approx 30^{\circ}\right)$, but is mostly a result of the change in surface free energy $\left(\Delta \theta_{\text {icenucleus }}\right) . \ldots \ldots$. . . . . . . . 102

\subsection{Freezing temperature $\left(T_{\text {Freeze }}\right)$ distributions from our surface texture} study Freezing temperatures for crystallization originating at the substrate interface have a similar distribution to our earlier experiments (Figure 6.3). Similarly, the few occurrences of immersion freezing on the fiber have a very small variance. Nucleation events beginning on the fiber contact line, exhibit a very large variance. A shift in the mean temperature for contact and immersion freezes $(\Delta t \approx 2 K)$ is in line with earlier observation of contact nucleations $(\Delta T=2-5 K)[19,68,23] \ldots \ldots 104$

6.5 A droplet rests on two regions on a silicon substrate. The darker region of the substrate is smooth, while a micro surface texture (Figure 5.5) has been applied to the lighter region as described in Chapter 5. During repeated freeze-thaw cycles, crystallization was initiated on both regions, indicating no preference towards the micro textured region. . . . . . . . . . . . . 107

6.6 Poking the droplet Here an electro-chemically sharpened tungsten tip is brought into contact with a spherical cap droplet (top left). Nucleation is initiated on the hydrophobic substrate, away from the probe (Right). SEM analysis of the probe tip reveals a blunted tip and course surface features $(r>100 n m) \ldots \ldots \ldots \ldots \ldots \ldots$ 
6.7 An example of nucleation occurring ahead of the crystallization front. Here nucleation is induced by an external crystal in a "Cooper mode" event (yellow circle in frame 1). Crystallization of the droplet proceeds from the nucleation site, but new bar shaped ice crystals appear to form ahead of the crystallization front (yellow circle frame 5). When viewed at full speed it is clear that a spherical wave, originating from the catalyst site, precedes the nucleation of these new crystals. . . . . . . . . . . . . 111

6.8 A rare example of nucleation induced by bursting of a bubble. A film strip shows the crystallization beginning at the top of a droplet, with several bubble trapped at the apex. A closer examination (right), reveals a decrease in the number of bubbles between before and after the formation of the crystal. When played in at full speed, it is clear that the nucleation is triggered by the bursting of one or more air bubbles. . . . . . . . . . . 113 


\section{Acknowledgments}

This document details my attempts to broaden our collective knowledge of physical pathways, in an attempt to better clarify my role as the man who freezes water. I am eternally burdened with a debt of gratitude to my advisors Dr. Raymond Shaw, and Dr. Alex Kostinski. Together they transformed my understanding of science, forcing me to approach problems in new ways and gave me many practical tips, like how to leave enough minor errors in a manuscript to give reviewers something to pick at... I hope to emulate their examples in my life to be a better scientist and man.

I would also be remiss not to thank my research group and collaborators for all their assistance, and camaraderie. It was my honor to work with such an amazing group of young scientists (Dr. Matt Beals, Dr. Kelken Chang, Dr. Jiang Lu, Dr. Dennis Niedermeier, Dr. Jacob Fugal, Mr. Nick Black, Ms. Nelly Iceta, Mr. Neel Desai, Mr. Fan Yang, Mr. Dave Ciochetto, Mr. Joe Niehaus, Mr. Kris Bunker, Mr. Joe Charnawskas, Mr. Adam Laxo). My time at MTU was made immeasurably better by the outstanding staff of the Physics department, especially its head Dr. Ravi Pandey. I should also recognize the benevolence of the groups which helped to fund all of my endeavors. Thanks to the MTU Physics Department, the MTU Graduate School, the Department of Energy, NASA and the Michigan Space Grant Consortium. 
Lastly, I wish to thank those without whom I would not have been able to complete this journey. I have always been blessed with the unwavering support of my family, and must pay special thanks to my sister Greer who first introduced me to MTU. MTU provided me a great education, but more importantly it brought me the love of my life. I leave MTU a proud husband to Renee Gurganus and father to Colin Alexander Gurganus. My last acknowledgment is reserved for my darling wife, none of this would be possible without your boundless love and gentle prodding. You pull me back to the ground when my head gets stuck in the clouds. 


\section{Preface}

In this document I present results from studies preformed in the Cloud Physics Lab at MTU. These results (detailed in Chapters 3, 4 and 5) have been previously published $[31,32,30]$ in peer reviewed articles and presented at professional conferences. The composing of these articles was accomplished through the collaborative effort of myself and my dissertation advisors Dr. Raymond Shaw and Dr. Alex Kostinski, with all parties contributing equally to the drafting and editing of the text. I was particularly responsible for taking and analyzing experimental data as well as generating figures for the articles, though I was assisted in the former task by Mr. Joseph Charnawskas for the experiments detailed in Chapter 5. These individuals are noted in the published works as co-authors, and I would like to again thank the wholeheartedly them for their efforts. 



\section{Abstract}

While nucleation of solids in supercooled liquids is ubiquitous[15, 65, 66], surface crystallization, the tendency for freezing to begin preferentially at the liquid-gas interface, has remained puzzling[74, 18, 68, 69, 51, 64, 72, 16]. Here we employ high-speed imaging of supercooled water drops to study the phenomenon of heterogeneous surface crystallization. Our geometry avoids the "point-like contact" of prior experiments by providing a simple, symmetric contact line (triple line defined by the substrate-liquid-air interface) for a drop resting on a homogeneous silicon substrate. We examine three possible mechanisms that might explain these laboratory observations: (i) Line Tension at the triple line, (ii) Thermal Gradients within the droplets and (iii) Surface Texture.

In our first study we record nearly perfect spatial uniformity in the immersed (liquid-substrate) region and, thereby, no preference for nucleation at the triple line. In our second study, no influence of thermal gradients on the preference for freezing at the triple line was observed. Motivated by the conjectured importance of line tension $(\tau)$ $[1,66]$ for heterogeneous nucleation, we also searched for evidence of a transition to surface crystallization at length scales on the order of $\delta \sim \tau / \sigma$, where $\sigma$ is the surface tension[14]; poorly constrained $\tau$ [49] leads to $\delta$ ranging from microns to nanometers. 
We demonstrate that nano-scale texture causes a shift in the nucleation to the three-phase contact line, while micro-scale texture does not. The possibility of a critical length scale has implications for the effectiveness of nucleation catalysts, including formation of ice in atmospheric clouds[7]. 


\section{Chapter 1}

\section{Background}

\subsection{Atmospheric Nucleation}

Clouds play an important role in global radiative balance and precipitation. With sizes ranging from several meters to many kilometers, it is startling to realize that clouds are actually composed of innumerable $\sim 10 \mu \mathrm{m}$ droplets, with each governed by thermodynamics, turbulence and complex interactions with ice, aerosol particles and other droplets. Understanding these processes represents a fundamental challenge to our understanding climate and weather fluctuations. Sophisticated remote sensing satellites monitor cloud structure and properties on a global scale and decades of these observations aid in the development of parametrizations for climate models to clarify variability in the atmosphere. Despite the complexities of these tools, a poor understanding of the underlying microphysical cloud processes limits the fidelity of climate and weather models at all scales $[53,47]$. 
The efforts described in this dissertation are aimed at better understanding one piece of this complex puzzle: the formation of ice in supercooled water. In clouds, the freezing of supercooled water can initiate precipitation processes and drive cloud motions and turbulent mixing through the release of latent heat. The formation of ice within supercooled cloud droplets in the lower troposphere is dominated by heterogeneous nucleation due to the abundance of natural and anthropogenic aerosol catalyst particles[7]. Disentangling the many interacting processes in natural clouds by isolating just the freezing process in the laboratory is important for development of a fundamental understanding necessary for correct parameterization in cloud microphysical models.

\subsection{Nucleation Theory}

Crystallization is initiated by the formation of an initial crystal from a metastable liquid. If the initial crystal or "seed" is of the correct size then it will grow spontaneously, with the total water volume freezing at a rate limited by the dissipation of the latent heat of freezing. Once all of the surfaces of the initial volume have undergone phase change, the volume will begin crystallize, but at a slower rate. Latent heat released during this process will cause the liquid temperature to heat to the melting point, until the volume has finished its transformation. In the experiments described here we exploit these properties to examine the role of heterogeneous nucleation of ice in spherical cap droplets (for a detailed explanation see Section 2.2).

Perhaps the least understood piece of the crystallization puzzle is the formation of the initial seed crystal that initiates the process. It has long been observed that nucleation 
is dependent on temperature, with many liquids freezing without external catalyst at a specific homogeneous nucleation temperature $\left(T_{\text {Homo,Water }} \approx-38^{\circ} \mathrm{C}[61]\right)$. Similarly, we know from empirical studies of heterogeneous nucleation that some catalyst materials can

significantly raise the mean freezing temperature $\left(T_{\text {Melt }}>T_{\text {Heterogeneous }}>T_{\text {Homogeneous }}\right)$ and it has been common in the literature to characterize a catalyst material by a nucleation temperature. In fact, freezing temperatures exhibit some distribution around the mean, and nucleation is more precisely described by a probability of freezing. In this context, freezing is described by a specific nucleation rate which is a function of Temperature $(j(T))$. For homogeneous nucleation the probability of freezing is a function of the homogeneous nucleation rate $\left(j_{\text {homo }}\right)$ and time $(t)$ and the volume of the nucleus

$$
P_{\text {homo }}=1-e^{-j_{\text {homo }} V t} .
$$

Similarly, for heterogeneous nucleation the probability of freezing is a function of the heterogeneous nucleation rate $\left(J_{h e t}\right)$, time $(t)$ and catalyst surface area

$$
P_{h e t}=1-e^{-j_{h e t} A t} .
$$

These processes are shown schematically in Figure 1.1.

\subsubsection{Nucleation Rate}

The nucleation rate $\left(j_{h e t}\right)$ is composed of three temperature dependent terms [61]

$$
j_{h e t}=\frac{k T}{h} * \exp \left(\frac{-\Delta G^{*}}{k T}\right) * n_{s} \exp \left(\frac{-\Delta F}{k T}\right) .
$$




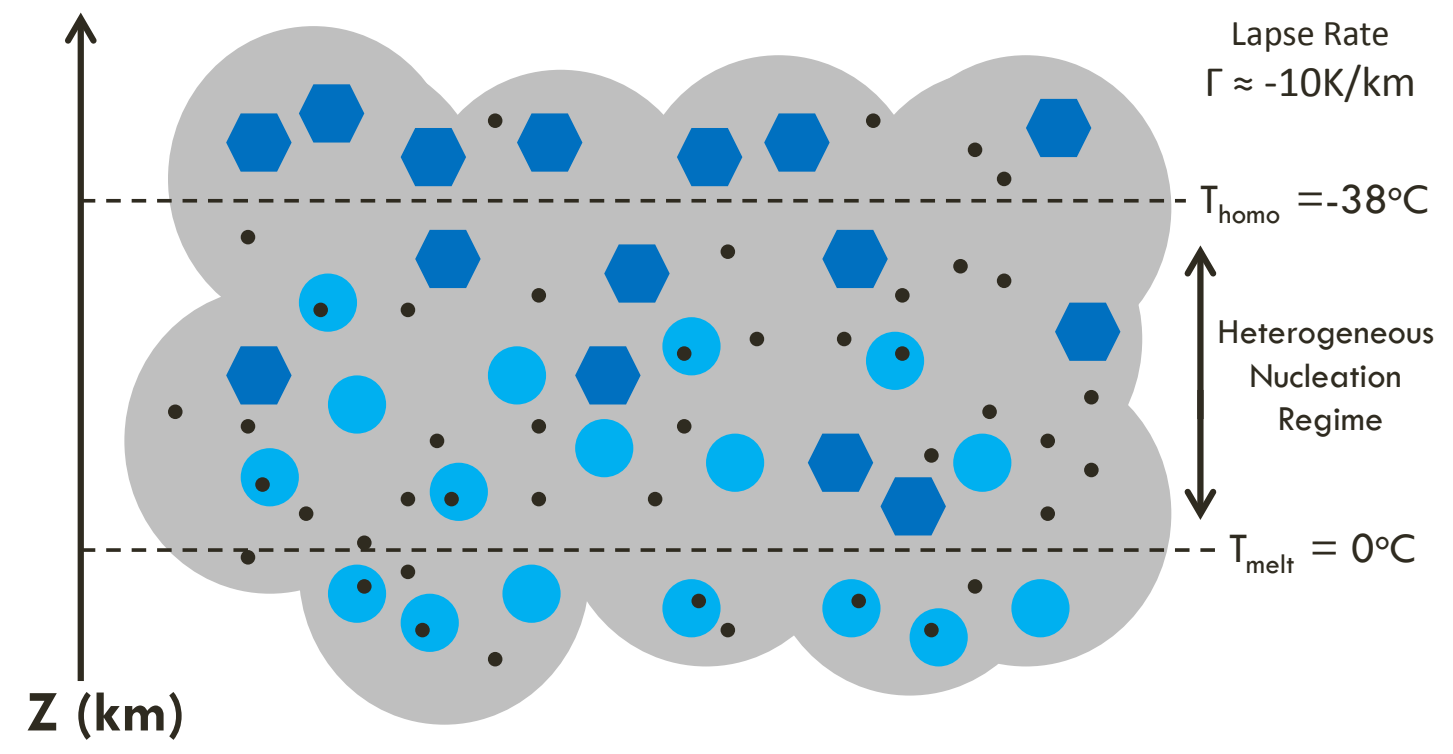

Water Droplets

Ice Crystals

- Aerosol Particles (CCN and IN)

Figure 1.1: Nucleation of Modes in Tropospheric Clouds Homogeneous and heterogeneous nucleation of cloud droplets can occur at different elevations in tropospheric clouds. A rising air parcel will be cooled at the atmospheric lapse rate $\Gamma<10 \mathrm{~K} / \mathrm{km}$, raising the droplets into the heterogeneous and homogeneous regimes respectively $\left(T_{\text {Melt }}>\right.$ $\left.T_{\text {Heterogeneous }}>T_{\text {Homogeneous }}\right)$. Above the heterogeneous freezing threshold, supercooled water droplets and ice particles can exist simultaneously in a "mixed-phase" cloud. The largest ice particles, not necessarily formed by homogenous nucleation, grow via the Bergeron process or conglomeration until they become to heavy to be transported by internal cloud eddys and fall out of the cloud as precipitation.

The first two terms $\frac{k T}{h} * \exp \left(\frac{-\Delta G^{*}}{k T}\right)$ originate from the Eyring Equation in reaction rate theory [22], which is basically the classical Arrehnius equation with the molecular vibration frequency $\left(\frac{k T}{h}\right)$ chosen as the prefactor for a Boltzman term ${ }^{1}$. The Gibbs energy barrier $(\Delta \mathrm{G})$ arises from the need to form a new ice-water interface around the nucleus and will be discussed in more detail in the next section. The third term contains another Boltzman factor, but in this case the energy barrier is the activation energy for water

${ }^{1}$ This time constant is still debated, and nucleation might possibly be be more accurately described by a non-quantum mechanical time contact such as the Debye frequency 
monomers binding to the ice nucleus. The prefactor $\left(n_{s}\right)$ is the density of water molecules in the vicinity of the catalyst surface. This rate can be thought of as the product of two probabilities, first the probability of achieving a nucleus of a critical number of monomers and the second as the probability of adding monomers to the critical nucleus.

The sensitivity of nucleation to temperature becomes immediately clear when equation

1.3 is substituted into equation 1.2 , with $\mathrm{P}_{\text {het }} \propto \exp \left(-\exp \left(-\frac{1}{T}\right)\right)$. We will see later (equation 1.10) that the nucleation is even more sensitive to temperature, with $\Delta G^{*} \propto \frac{1}{\Delta T^{2}}$, where $\Delta T$ is the supercooling temperature $\left(\Delta T=T_{m e l t}-T\right)$. This helps to explain the relatively narrow distribution of freezing temperatures observed in most experiments. This implies that large shifts in the freezing temperature might more readily be explained by lowering of the energy thresholds in equation $1.3(\Delta G$ or $\Delta F)$, than by increasing the other parameters in equation $1.2(A$ and $t)$. To provide a possible explanation for the shift in freezing temperature observed for some systems we will examine a mechanism for lowering of the Gibbs energy barrier $\Delta G^{*}$ in section 1.4 .

\subsubsection{Gibbs Barrier}

\subsubsection{Homogenous Nucleation}

Gibbs [25] was the first to propose the idea that an energy barrier is associated with the formation of a new interface. The energy barrier $\Delta G$ is a sum of two terms, the first is proportional to the volume of the nucleus and the second is proportional to surface area of the nucleus (Equation 1.4). In the simpler homogenous case, the nucleus is assumed to be 
spherical.

$$
\Delta G_{\text {homogenous }}=A * \frac{4 \pi}{3} r^{3}+B * 4 \pi r^{2}
$$

The prefactor of the volume term $(A)$ describes the energy contribution from the difference in chemical potentials $\Delta \mu_{\text {Ice-Liquid }}$ while the term $B$ describes the free energy from interface created and is a function of the surface tension $\left(\sigma_{\text {Ice-Liquid }}\right)$ of the nucleus:

$$
A=n_{\text {Ice }} * \Delta \mu_{\text {Ice-Liquid }} \quad B=\sigma_{\text {Ice-Liquid }}
$$

with units of $\frac{J}{m^{3}}$ and $\frac{J}{m^{2}}$ respectively, where $\rho_{I c e}$ is the number density of molecules in the nucleus. When the liquid is in a metastable state (supercooled) the ice phase is energetically preferred such that $\Delta \mu_{\text {Ice-Liquid }}<0$. This leads to a negative contribution from the volume term in $\Delta G$.

In order to transform the $A$ term into a more useful factor, we will convert the difference in chemical potential to a function of temperature $(T)$ and water vapor saturation ratio $\left(S=\frac{p}{p_{s a t}}\right)$,

$$
\Delta \mu_{\text {Ice-Liquid }}=k T \ln (S)
$$

where $k$ is Boltzman's constant [44]. The vapor saturation can be also be represented as a function of the supercooling temperature $(\Delta T)$

$$
S=\exp ^{\left(\frac{l_{f}}{k T} \frac{\Delta T}{T_{m e l t}}\right)}
$$


where $l_{f}$ is the latent heat of fusion and $T_{\text {melt }}$ is the melting temperature of the system $\left(T_{m e l t}=273 \mathrm{~K}\right.$ for water). Using these steps, the difference in chemical potential can now be written simply as a function of temperature.

$$
\Delta \mu_{\text {Ice-Liquid }}=\frac{l_{f} \Delta T}{T_{m e l t}}
$$

The prefactor $A$ is now a linear function with supercooling temperature. The density of water is also a weakly varying function of $T$, but will be considered constant,

$$
A=\rho_{\text {ice }} \frac{l_{f} \Delta T}{T_{\text {melt }}} \quad \text { and } \quad B=\sigma_{\text {Ice-Liquid }}
$$

To solve for the critical nucleus size $\left(r^{*}\right)$ we take the derivative of $\Delta G$ with respect to nucleus size and set it equal to zero $\left(\frac{d \Delta G}{d r}=0\right)$ to find the top of the energy barrier. From this we can solve for $r$ to find the critical nucleus size $r^{*}=-\frac{2 B}{A}$. Plugging this result back into equation 1.4 yields the critical energy barrier for nucleation

$$
\Delta G_{\text {homogenous }}^{*}=\frac{16 B^{3}}{3 A^{2}} \quad \text { or } \quad \Delta G_{\text {homogenous }}^{*} \propto \frac{1}{\Delta T^{2}} .
$$

\subsubsection{Heterogeneous Nucleation}

In the presence of a nucleation catalyst we assume that the nucleus will form in the shape of a spherical cap (Figure 1.2). This shape is convenient due to the macroscopic notion of surface tension. In this geometry we introduce a material dependent variable contact angle $(\theta)$, which describes the ability of a droplet to wet a surface or, for an ice crystal the affinity for ice to form on a substrate. For a perfect sphere $\theta=2 \pi$, and $\theta=\pi$ for a hemispherical 


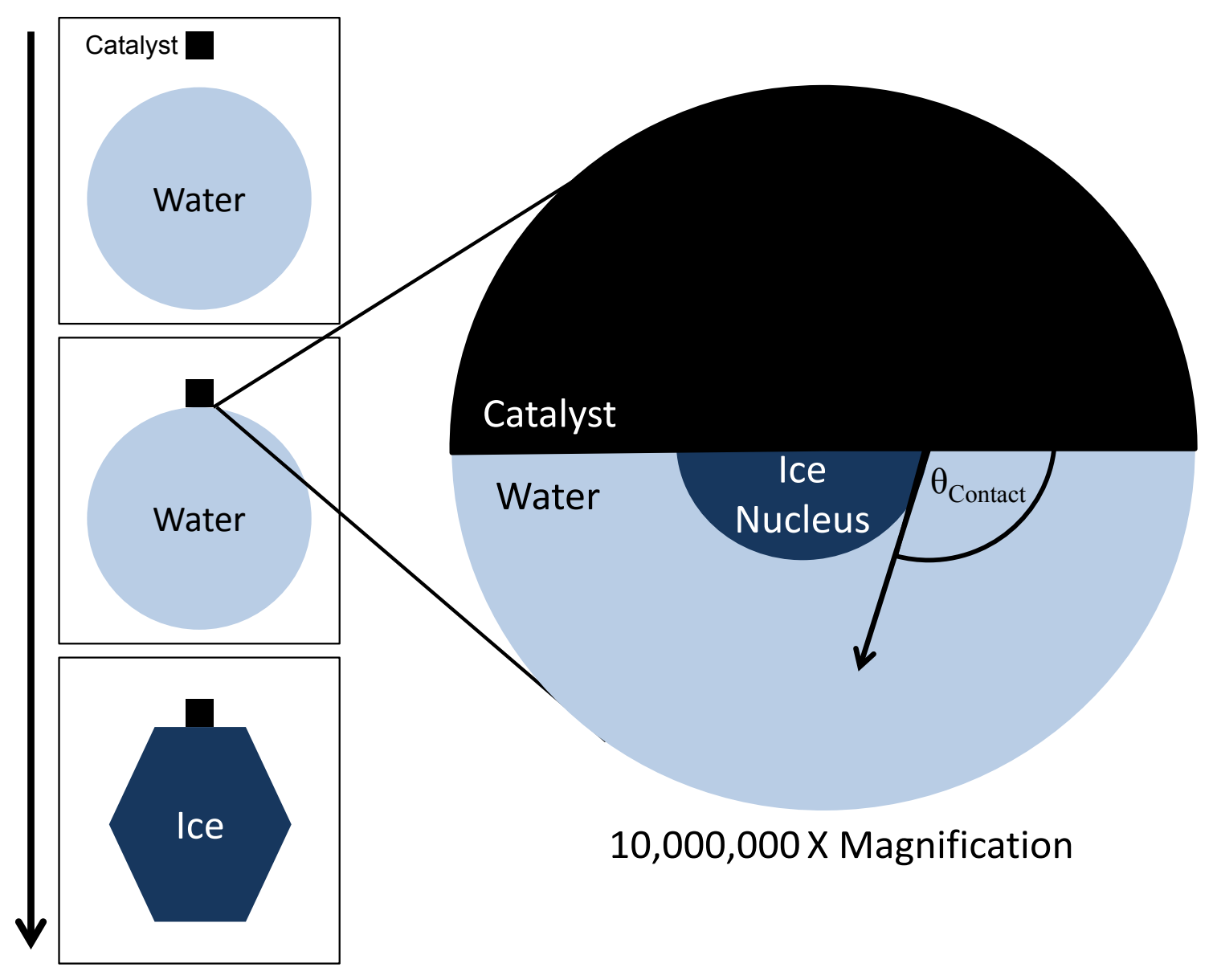

Figure 1.2: Formation of Nucleus on External Catalyst: A schematic of ice formation on an catalyst particle. Before crystallization of the entire droplet can proceed, a spherical cap nucleus $\left(r_{\text {nucleus }}>r_{\text {critical }}\right)$ must be formed. Crystallization will then spread out from this location until the entire droplet is frozen. In this situation the nucleus forms at the two phase-interface, and would be considered immersion mode nucleation.

cap. Materials with higher contact angles for ice are said to be ice-phobic and tend to be poor candidates for ice nucleation catalysts.

When comparing the size of the critical nucleus in the spherical cap geometry to that of a spherical nucleus, it is easy to see the catalyst can lower the Gibbs energy barrier for nucleation by effectively reducing the number of monomers required to reach the critical 
size. By altering equation 1.4 to include the change in volume and surface areas of the new geometry, a simple relation arises for the height of the critical energy barrier

$$
\Delta G_{\text {heterogenous }}^{*}=\Delta G_{\text {homogenous }}^{*} * f\left(\cos \theta_{\text {ice }}\right)
$$

that varies from the homogenous case as a function of the the cosine of the contact angle [44]

$$
f(\cos \theta)=\frac{(2+\cos \theta)(1-\cos \theta)^{2}}{4}
$$

To arrive at this result, it was necessary to compare the surface tension of the ice-water interface $\left(\sigma_{\text {Ice-Liquid }}\right)$, which is the only surface tension in $\Delta G_{\text {homogenous }}^{*}$, to the surface tension of the new catalyst-ice interface at the base of the spherical cap $\left(\sigma_{\text {Catalyst-Ice }}\right)$. This is accomplished through Young's Equation

$$
\sigma_{\text {Ice-Liquid }} * \cos \theta=\sigma_{\text {Catalyst-Liquid }}-\sigma_{\text {Catalyst-Ice }} .
$$

Because of the difficulty in measuring the contact angle of a spherical cap ice nucleus in a supercooled liquid, the contact angle is often left as a fit parameter in experiments[55]. Despite this complication, it is clear from equation 1.11 that ice-phillic materials will significantly reduce the energy barrier for nucleation. This result implies that heterogeneous nucleation will be the dominant nucleation mechanism in the presence of catalyst particles with low contact angles. 


\subsubsection{Line Tension}

While the idea of a Gibbs energy barrier is well accepted for nucleation theory, recent experiments and simulations suggest that this simple formulation (Equation 1.4) may be incomplete $[35,1]$. These authors suggest that the inclusion of a third term linear in seed radius $(\propto r)$ with a prefactor $(\tau)$ allows for a much closer fit with between data and theory. The idea of an energy associated with the discontinuity at the three phase interface line was first proposed by Gibbs [25] over a century ago. This "line tension" term $(\tau)$ was first included in nucleation by Gretz in 1966 [28], who extended upon the idea of edge energies at mono-atomic edges introduced by Hirth and Pound [37]. In the simplest case of a hemispherical cap nucleus, the line tension energy is proportional to the length of triple line at the perimeter of a droplet $\left(l_{\text {perimeter }}=2 \pi r\right)$. This new term is represented in equation 1.14 by the third term $\propto r$, where $\mathrm{C}=\tau$, again a hemispherical cap for simplicity,

$$
\Delta G_{\text {hemisphericalcap }}=A * \frac{2 \pi}{3} r^{3}+B * 2 \pi r^{2}+C * 2 \pi r .
$$

The importance of this term is evident in Figure 1.3, with the height of the energy barrier $\Delta \mathrm{G}$ lowering significantly when line tension (term C) is taken into account.

To understand the importance of this new term to the Gibbs energy barrier, we need to quantify the value of $\tau$. Line tension is believed to become significant only at small scales, much below the capillary length $\left(R_{\text {Capillary }}\right)[80]$

$$
R_{\text {Capillary }}=\sqrt{\frac{2 \sigma}{g \rho_{\text {water }}}}
$$




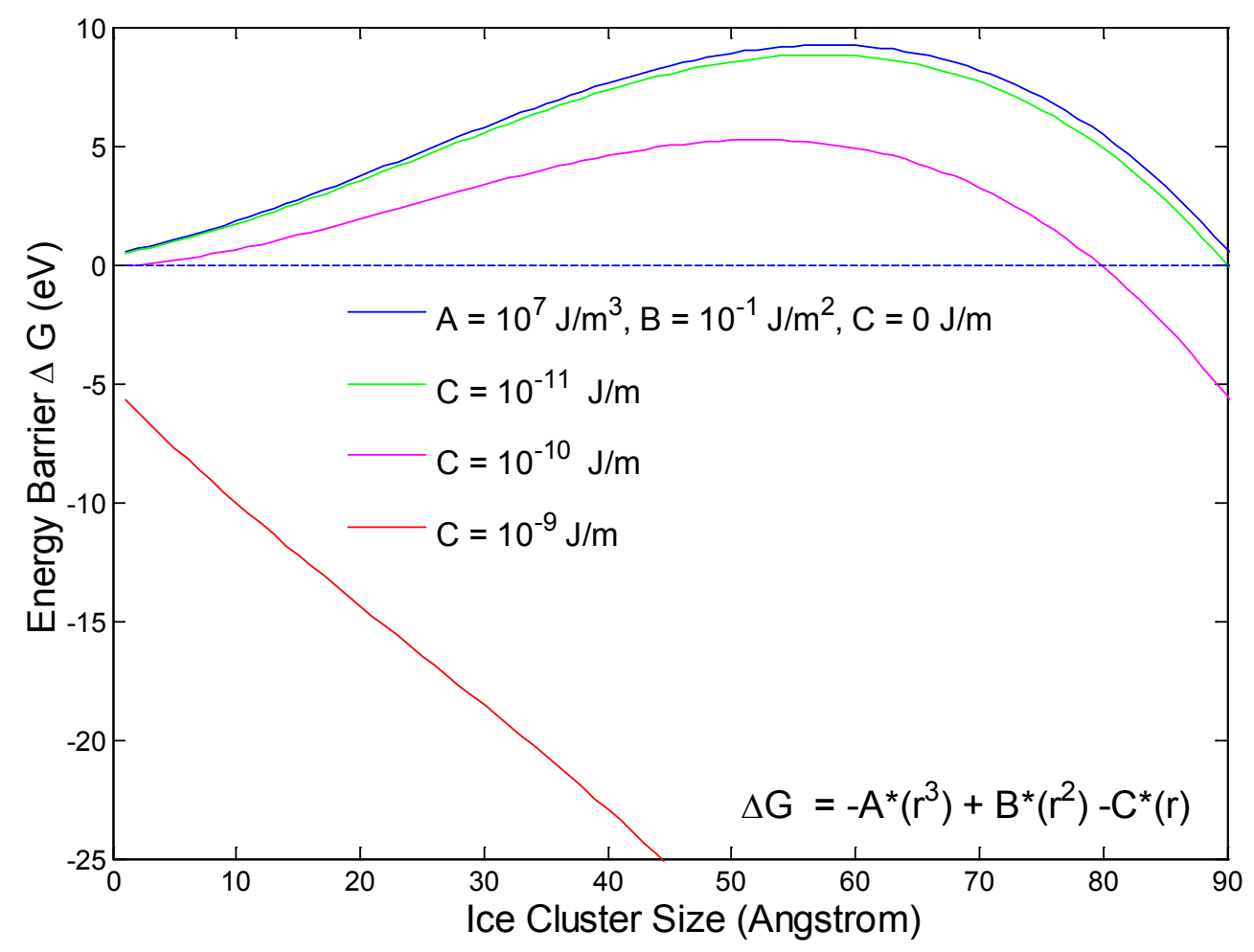

Figure 1.3: Examining the shape of the energy barrier to nucleation $\Delta \mathrm{G}$ (equation 1.14) when line tension (C) is included. Typical values for $\mathrm{A}$ and B derived from equation 1.9 with $l_{f} \sim 10^{8} \mathrm{~J} / \mathrm{m}^{3}$ and $\sigma_{\text {ice-water }} \sim 10^{-1}$. The three lower lines (green, pink orange) represent increasing values of line tension. For line tension values greater than $|\tau|=10^{-9}$ the barrier to nucleation no longer exists, which suggests that this is a fundamental limit to the magnitude of $\tau$.

for water, and will lead to a change in contact angle between macroscopic $\left(\theta_{\text {macro }}\right)$ and microscopic liquid droplets $\left(\theta_{\text {micro }}\right)$. This relationship is described by a modified Young's Equation $[49,50]$.

$$
\cos \left(\theta_{\text {macro }}\right)=\cos \left(\theta_{\text {micro }}\right)-\frac{\tau}{\sigma_{\text {Liquid }- \text { Vapor }} * r}
$$

Measuring the change in contact angles with droplet size $\left(\Delta \theta=\theta_{\text {macro }}-\theta_{\text {micro }}\right)$, allows for a direct measurement of $\tau$, assuming that $\sigma$ is known. A wide variety of values in 
the literature $\left(10^{-6}-10^{-11} \mathrm{~J} / \mathrm{m}\right)[49,50]$ reflect the large uncertainty of $\tau$. DeGennes notes that many of these values (measured optically) are uncertain to three orders of magnitude [14], as a result of the difference between the size of the droplets $(\sim \mathrm{mm})$ and the wavelength of light $(\sim \mu \mathrm{m})$. More recently, AFM measurements of fullerene droplets put

the bound at the lower end of this range $\left(10^{-10}-10^{-11} \mathrm{~J} / \mathrm{m}\right)[5]$. This range agrees better with nucleation experiments [35], and the practical limit for $\Delta \mathrm{G}$ (below which no barrier to nucleation would exist) from figure 1.3.

Line tension seems to be a reasonable addition to classical nucleation theory, but unlike concepts like surface tension there is no underlying theoretical explanation for the existence of line tension[79]. To this point, it has been assumed since 1966 [28] that it acts to decrease the contact angle of a ice nucleus $(C<0$ in equation 1.14), thereby lowering $\Delta \mathrm{G}$. The opposite trend is observed in many measurements of $\tau$, leading to large uncertainties in both the magnitude and sign of line tension. In chapter 5 we will examine how line tension may be important for contact nucleation, but it is important to note that other mechanisms might be responsible for observed conflicts between theory and measurements.

\subsection{Homogeneous Surface Crystallization}

Homogenous surface crystallization, where nucleation prefers the liquid-vapor interface and scales with the surface area of a liquid droplet, has been proposed as a relevant mechanism for freezing of water droplets that are found in supercooled tropospheric clouds [74]. This effect may be more universal, with evidence observed for a variety of nucleation processes, from atomic liquids[51], salts[2], tetrahedral liquids[45], hexaflourides[11], 
metal alloys[69, 72], Nickel-Silicon[48] and polymers[8]. The probability of freezing then becomes a function of two specific nucleation rates $j_{\text {volume }}$ and $j_{\text {surface }}$

$$
P_{\text {freeze }}=1-e^{-\left(j_{\text {volume }} V+j_{\text {surface }} A\right) t}
$$

where we would expect, assuming $j_{\text {volume }} \approx j_{\text {surface }}$, the surface term to dominate at small

length scales owing to the large surface to volume ratio $\left(\frac{j_{\text {Surface }} A}{j_{\text {Volume }} V} \propto \frac{1}{r}\right)$. Recent experiments with isolated spherical droplets [18] note no role for surface freezing in droplets with $d>10 \mu \mathrm{m}$. The authors speculate that this does not preclude the existence of surface freezing at smaller scales, but working with individual drops this size is a challenge in the laboratory. Despite this null result for homogeneous droplets, there is strong evidence that surface crystallization may play an important role in the heterogeneous mode where a catalyst particle comes into contact with a droplet interface[68, 19, 23]. It has been speculated that this could be related to the phenomenon of contact nucleation.

\subsection{Heterogeneous Surface Crystallization (Contact Nucleation)}

Heterogeneous nucelation of ice in a supercooled liquid for atmospheric clouds is typically confined to two different mechanisms, "contact nucleation" with an aerosol particle coming into contact with a droplet interface (Figure 1.4 B, C, D) and "immersion nucleation" where the immersed solid aerosol in the droplet volume induces freezing (Figure 1.4 A). Laboratory experiments have demonstrated a preference for nucleation in the contact mode with freezing temperatures typically $2-5 \mathrm{~K}$ warmer than in the immersion 

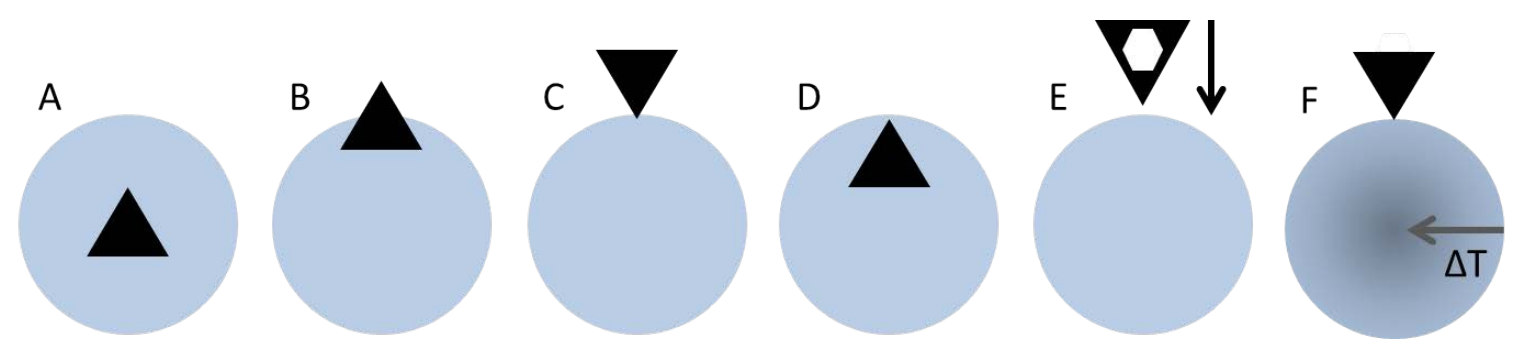

Figure 1.4: Examples of the various scenarios for droplet-particle (triangles) interactions. A. Fully immersed particle B. Partially immersed particle C. Point-Like contact of particle at droplet interface D. Point-Like contact "Inside-Out" E. A particle with an ice crystal on the surface coming into contact with the droplet $\mathbf{F}$. A particle coming into contact with a droplet that is coldest at the interface due to rapid cooling.

mode $[7,19,68,23,56]$. If this effect is relevant for atmospheric nucleation, a five degree shift in the freezing temperature for superooled drops may drive precipitation formation to lower altitudes due to the tropospheric lapse rate $\Gamma \approx 10 \mathrm{~K} / \mathrm{km}$ [27]. Ice formed at higher temperatures $\left(\Delta T_{\text {Contact }}\right)$ may grow more rapidly due to increased vapor pressure $\left(\Delta p_{\text {vapor }} \propto e^{-\Delta T_{\text {Contact }}^{-1}}\right)$ [27] forcing a reevaluation of contact nucleation in modern cloud models. While this result has large implications for cloud formation and structure, the reason and relevance for this significant difference in the energy barrier to nucleation is an open question. Here, we will outline three possible mechanisms that might explain these laboratory observations: (i) induction by external crystal, (ii) thermal gradients, (iii) line tension and (iv) surface texture.

\subsubsection{Nucleation by External Crystal "Cooper mode"}

The first mechanism, proposed in 1974 by Cooper [13], asserts that a dry aerosol particle that comes into contact with a cloud droplet might contain an ice crystal (Figure 1.4 E). Because the particle would need to be hydrophobic, the ice crystal would likely be formed 
by direct deposition from vapor, bypassing the liquid phase. The ice embro might be of sub-critical size for deposition mode freezing, but of critical size for immersion freezing. This mechanism is easy to visualize on a macroscopic scale, if you were to drop an ice cube into a glass of supercooled water, the latter would immediately begin to freeze (try it, it's fun).

Despite the elegance and simplicity of this mechanism, it is difficult to imagine a scenario when it would be practical in the troposphere. Unfortunately, deposition nucleation is usually only relevant at temperatures approaching that of homogenous nucleation $(\mathrm{T} \approx$ $-38^{\circ} \mathrm{C}$ ), and for low supersaturation values where liquid water is unlikely to form [39]. Additionally, experiments with fully immersed particles indicate that contact nucleation can occur if the particle comes into contact with the droplet interface $[19,68,23]$. This "Inside-Out" freezing precludes contact with an external crystal, which suggests some other mechanism is likely responsible.

\subsubsection{Thermal Gradients}

Another possible explanation for this phenomena is that it might be an artifact of laboratory experiments. Many experiments rely on a continuous cooling technique to record a freezing temperature $[52,23,19,73]$, and this technique may lead to a thermal gradient developing within a droplet. In that scenario, the surface of the droplet can be cooler than the interior, in which case one would expect to observe surface crystallization (Figure 1.4 F). 
As discussed later in Chapters 3 and 4, the size of this gradient can be estimated with two system parameters: cooling rate $\lambda=\frac{\partial T}{\partial t}$ and droplet size $d$. The diffusion of heat is governed by Fick's Law (assuming radial symmetry) $\frac{\partial T}{\partial t}=\chi \frac{\partial^{2} T}{\partial r^{2}}$. Taking the droplet size as the characteristic length of the system, we can define a time scale for diffusion as $\tau_{\text {diffusion }}=\frac{d^{2}}{\chi}$. Multiplying this time scale by the system cooling rate, we arrive at characteristic gradient for the system $(\Delta \mathrm{T})$.

$$
\Delta T \approx \lambda \tau=\lambda * \frac{d^{2}}{\chi}
$$

Analysis of equation 1.18 reveals that strong cooling rates or large droplets might contribute to a large gradient. To date, experiments that are able to observe a trend towards contact nucleation focus on larger droplets than are commonly found in the atmosphere $\left(r_{\text {drop }}>100 \mu m\right)$ because they are easier to generate and observe. These drops are ideal for a laboratory setting because they will evaporate more slowly than droplets with smaller radii of curvature due to decreased vapor pressure (Kelvin relation[27]). The droplet sizes are also typically selected to be larger than the impacting particle, so that the shape of the droplet is not affected by the wetting of the particle. The use of slow cooling rates might help mitigate this problem, but thermal gradients may be important in some experiments.

It is also important to note that thermal gradients might also play a role in cloud processes. For a typical cloud droplet $(d \sim 10 \mu \mathrm{m})$, undergoing a strong updraft of $10 \mathrm{~m} / \mathrm{sec}$ and a lapse rate of $10 \mathrm{~K} / \mathrm{km}$ would correspond to a cooling rate of $\lambda=0.1 \mathrm{~K} / \mathrm{sec}$. In this scenario the the thermal gradient within the droplet would be smaller than a typical thermal measurement precision $\left(\Delta T \approx 10^{-3} K\right)$. But while the rapid cooling in updrafts is expected to be far less important than its analog in the laboratory, surface crystallization might still 
be biased by evaporative cooling of the interface. In mixing regions at cloud boundaries, entrainment of dry air can lead to rapid evaporation of cloud droplets which may create a strong bias towards surface crystallization. In this work we will address the possible role of thermal bias in laboratory experiments.

\subsubsection{Line Tension}

In the previous section we introduced line tension and demonstrated how it can effectively lower the threshold to nucleation. To demonstrate this effect, we we hope to explain how line tension might play a role in surface nucleation. We will consider two scenarios: (i) Homogeneous surface crystallization and (ii) Heterogeneous surface crystallization on a smooth catalyst substrate. For each case we will compare the energetic conditions required for surface nucleation to be preferred to immersion nucleation. To simplify the nucleation geometry we will assume that the critical nucleus is a cube, which is somewhat different from the spherical cap typically assumed in classical nucleation theory[61], or the hexagonal Wulff crystal used by Tabazadeh et al.[74]. The precise shape of the nucleus is unknown, so this work derivation is intended to give a conceptual understanding of the scaling of various terms.

\section{Homogeneous Surface Crystallization}

Here we examine the role of line tension $(\tau)$ for homogeneous surface crystallization. Our argument is based on the earlier work of Djikaev[74, 16], but here we use a simplified cubic geometry for the critical ice germ (Figure 1.5 left). The cubic crystal has a side length 

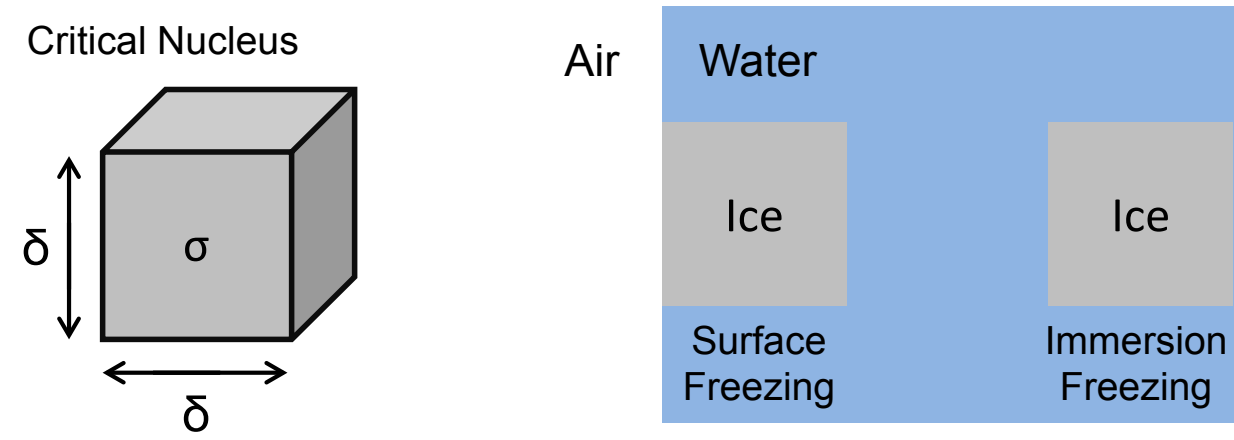

Figure 1.5: Homogeneous Nucleation: Volume vs. Surface To illustrate the difference between surface and volume nucleation, we consider homogeneous nucleation of a cubic critical nucleus with side length $\delta$. Each face of the crystal has a corresponding surface tension. For volume freezing all faces of the nucleus are in contact with water $\left(\sigma_{\text {Ice-Water }}\right)$, but in the surface configuration one of the faces is exposed to the air $\left(\sigma_{\text {Ice-Air }}\right)$. Additionally, the four edges surrounding the Ice-Air interface are in contact with all three phases (Ice, Air and Water) and will have a line tension energy $(\tau)$

$\delta$. Here we consider the formation of this crystal in both the liquid volume and at the air-water interface (Figure 1.5 right). To analyze which of these locations is preferred, we consider the the Gibbs free energy barrier $(\Delta G)$ for both configurations. In the immersion mode the difference in chemical potential between the stable ice and metastable liquid phases $(\Delta \mu)$, must be offset by the surface tension $(\sigma)$ associated with the six newly formed crystal faces

$$
\Delta G_{\text {Immersion }}=\delta^{3} \rho_{\text {Ice }} \Delta \mu_{\text {Ice-Water }}+6 \delta^{2} \sigma_{\text {Ice-Water }}
$$

where $\rho_{\text {Ice }}$ is the number density of ice molecules in the nucleus. These two terms are proportional to the crystal volume $\left(\delta^{3}\right)$, and surface area of a crystal face $\left(\delta^{2}\right)$ respectively. 
The Gibbs Barrier of the surface mode $\Delta G_{\text {Surface }}$ requires care because one of the six crystal faces is in contact with air $\left(\sigma_{\text {Ice-Air }}\right)$. This face replaces the a patch of the existing Water-Air interface, which has its own surface tension $\left(\sigma_{\text {Water-Air }}\right)$, and must be considered. Further, the edges of the exposed face are in contact with all three phases (air, water and ice) which requires that we consider a line tension energy $(\tau)$ for each exposed edge. The result is more complex than for the immersion mode, and we must consider five terms

$\Delta G_{\text {Surface }}=\delta^{3} \Delta \mu_{\text {Ice-Water }}+5 \delta^{2} \sigma_{\text {Ice-Water }}+\delta^{2} \sigma_{\text {Ice-Air }}-\delta^{2} \sigma_{\text {Water-Air }}+4 \delta \tau_{\text {Ice-Water-Air }}$

It is important to note that we have assumed that the size of the critical nucleus is identical for both surface and volume freezing, which is an oversimplification. Because the nucleation rate is a function of the Gibbs energy barrier $\left(j_{\text {nucleation }} \propto \exp \left[\frac{-\Delta G^{*}}{k T}\right]\right.$, for surface freezing to be preferred its energy barrier must be smaller

$$
\Delta G_{\text {Surface }}<\Delta G_{\text {Immersion }}
$$

To simplify this relation, we take the difference of the two values

$$
\Delta G_{\text {Surface }}-\Delta G_{\text {Immersion }}=\delta^{2} \sigma_{\text {Ice-Water }}-\delta^{2} \sigma_{\text {Ice-Air }}+\delta^{2} \sigma_{\text {Water-Air }}-4 \delta \tau_{\text {Ice-Water-Air }}
$$


For equation 1.21 to be satisfied the RHS must be negative, so this relation can be simplified to

$$
\sigma_{\text {Ice-Water }}>\sigma_{\text {Ice-Air }}-\sigma_{\text {Water-Air }}+\frac{4 \tau_{\text {Ice-Water }} \text { ir }}{\delta}
$$

The last term can be considered as a size dependent surface tension,

$$
\sigma_{\tau_{\text {Hото }}}=4 \frac{\tau_{\text {Ice-Water-Air }}}{\delta}
$$

and it is clear that its contribution becomes increasingly important as $\delta$ is decreased. This formulation is identical to that proposed by Tabazadah[74] if we neglect the existence of line tension $\left(\sigma_{\tau}=0\right)$. It remains an open question if this condition is satisfied for water.

If both modes are possible, then the probability of a droplet freezing $\left(P_{\text {freeze }}\right)$ becomes a function of the surface $\left(J_{\text {SurfaceNucleation }}\right)$ and volume nucleation rates $\left(J_{\text {VolumeNucleation }}\right)$

$$
P_{\text {freeze }}=1-e^{-\left(J_{\text {SurfaceNucleation }}+J_{\text {VolumeNucleation }}\right) t} .
$$

A possible explanation for this null result might be found in the relative magnitude of the surface area (A) and volume (V) contributions total nucleation rates

$$
\frac{J_{\text {SurfaceNucleation }}}{J_{\text {VolumeNucleation }}}=\frac{j_{\text {SurfaceNucleation }} A}{j_{\text {VolumeNucleation }} V} \propto \frac{1}{r_{\text {Drop }}} .
$$

This relationship suggests that exceedingly small droplet sizes might be necessary before a strong surface freezing contribution is evident in experiments, yet laboratory experiments have failed to observe a preference for homogenous surface nucleation in droplets as small as $r=10 \mu m$ [18]. Working with droplets of this size $\left(r_{\text {drop }}<1-10 \mu m\right)$, is difficult in the 
laboratory. Furthermore, due to the large uncertainty in the surface and line tension values, it is difficult to define a droplet size at which surface freezing may be relevant.

A candidate for the relevant length scale $(\delta)$ emerges from from the last term in equation 1.23. The contribution from this term must be negative for surface crystallization to reduce the value of the LHS. Recent measurements of $\tau$ suggest that this value is negative with an upper bound of $-10^{-10} J / m[5]$. Similarly, the last term must be of similar order to the other negative term $\left(\sigma_{\text {Water-Air }}\right)$ which leads to a simple relation for a relevant length scale $\delta$

$$
\frac{\tau_{\text {Ice-Water-Air }}}{\delta} \sim \sigma_{\text {Water-Air }} \quad \text { or } \quad \delta \sim \frac{\tau_{\text {Ice-Water-Air }}}{\sigma_{\text {Water-Air }}}
$$

From literature values of $\tau$ and $\sigma_{\text {Water-Air }}$, we can estimate the relevant length scale to be $\delta \sim 1-100 \mathrm{~nm}$. For homogeneous nucleation the critical nucleus is typically within this range (typically $r_{\text {critical }}<10 \mathrm{~nm}$ )), suggesting that line tension clould plausibly play a role. Because of the uncertainties in this estimate, it is very difficult to determine the surface nucleation rate $\left(j_{\text {surface }}\right)$, so more work is needed.

\section{Heterogeneous Surface Crystallization}

We will now extend the idea of line tension to the more general case of heterogeneous nucleation on a smooth catalyst substrate (Figure 1.6). This geometry is identical to that in the homogeneous case, except that in both the immersed and the surface configurations one of the crystal faces is in contact with the catalyst. The Gibbs barrier for the immersion 


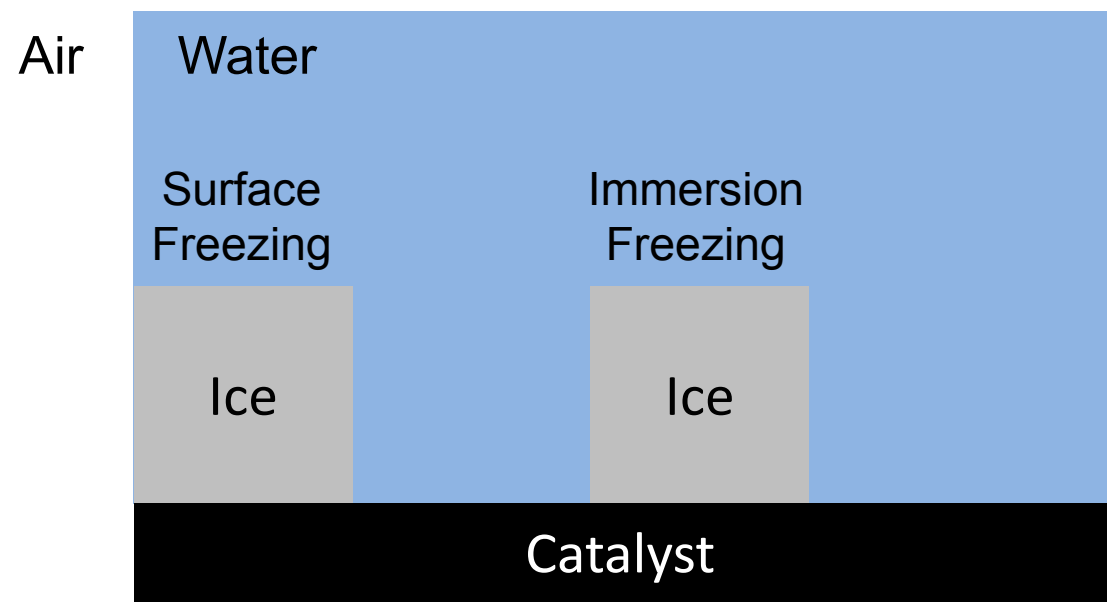

Figure 1.6: Heterogeneous Nucleation: Immersion vs. Surface To illustrate the difference between surface and immersion nucleation, we consider heterogeneous nucleation of a cubic critical nucleus with side length $\delta$. Each face of the crystal has a corresponding surface tension $\left(\sigma_{\text {face }}\right)$. This geometry is identical to that in Figure 1.5 except one face of either crystal is in contact with a catalyst substrate.

mode is

$$
\begin{array}{r}
\Delta G_{\text {Immersion }}=\delta^{3} \Delta \mu_{\text {Ice-Water }}+5 \delta^{2} \sigma_{\text {Ice-Water }} \\
+\delta^{2} \sigma_{\text {Ice-Catalyst }}-\delta^{2} \sigma_{\text {Water-Catalyst }}+4 \delta \tau_{\text {Ice-Water-Catalyst }}
\end{array}
$$

and for the surface mode it is

$$
\begin{aligned}
\Delta G_{\text {Surface }}= & \delta^{3} \Delta \mu_{\text {Ice-Water }}+4 \delta^{2} \sigma_{\text {Ice-Water }}+\delta^{2} \sigma_{\text {Ice-Air }}-\delta^{2} \sigma_{\text {Water-Air }} \\
& +\delta^{2} \sigma_{\text {Ice-Catalyst }}-\delta^{2} \sigma_{\text {Water-Catalyst }}+3 \delta \tau_{\text {Ice-Water-Catalyst }} \\
+ & 3 \delta \tau_{\text {Ice-Water-Air }}+\delta \tau_{\text {Ice-Air-Catalyst }}-\delta \tau_{\text {Water-Air-Catalyst }} .
\end{aligned}
$$

Unlike the homogeneous case, we are forced to introduce an additional three line tension terms here because the catalyst substrate introduces new three phase interfaces at the base of the nucleus in both configurations. 
If we follow the same procedure as with homogeneous nucleation in the previous section, then for Surface crystallization to be preferred (by equation 1.21) we arrive at a similar condition

$$
\begin{array}{r}
\sigma_{\text {Ice-Water }}>\sigma_{\text {Ice-Air }}-\sigma_{\text {Water-Air }}+3 \frac{\tau_{\text {Ice-Water-Air }}}{\delta}+\frac{\tau_{\text {Ice-Air-Catalyst }}}{\delta} \\
-\frac{\tau_{\text {Ice-Water-Catalyst }}}{\delta}-\frac{\tau_{\text {Water-Air-Catalyst }}}{\delta},
\end{array}
$$

which is of the same form as equation 1.23 , with

$\sigma_{\tau_{H e t}}=3 \frac{\tau_{\text {Ice }- \text { Water-Air }}}{\delta}+\frac{\tau_{\text {Ice }- \text { Air-Catalyst }}}{\delta}-\frac{\tau_{\text {Ice-Water-Catalyst }}}{\delta}-\frac{\tau_{\text {Water-Air-Catalyst }}}{\delta}$.

Here, the surface crystallization is dependent on the relative magnitude of four different line tension values, none of which are known.

\section{Size Dependence of Line Tension Contribution}

For both the homogeneous and heterogeneous surface crystallization scenarios described above the line tension contribution $\left(\sigma_{\tau}\right)$ to the Gibbs energy barrier is always proportional the inverse of the size of the nucleus

$$
\sigma_{\tau} \propto \frac{1}{r_{\text {nucleus }}}
$$

When we consider heterogeneous surface crystallization, four different line tensions must be considered but it is important to note that only one of these terms is present before nucleation $\tau_{\text {Water-Air-Catalyst }}$. If this term plays a significant role than a microscopic air-water-solid contact line must exist at the nucleation site when the droplet is still in 


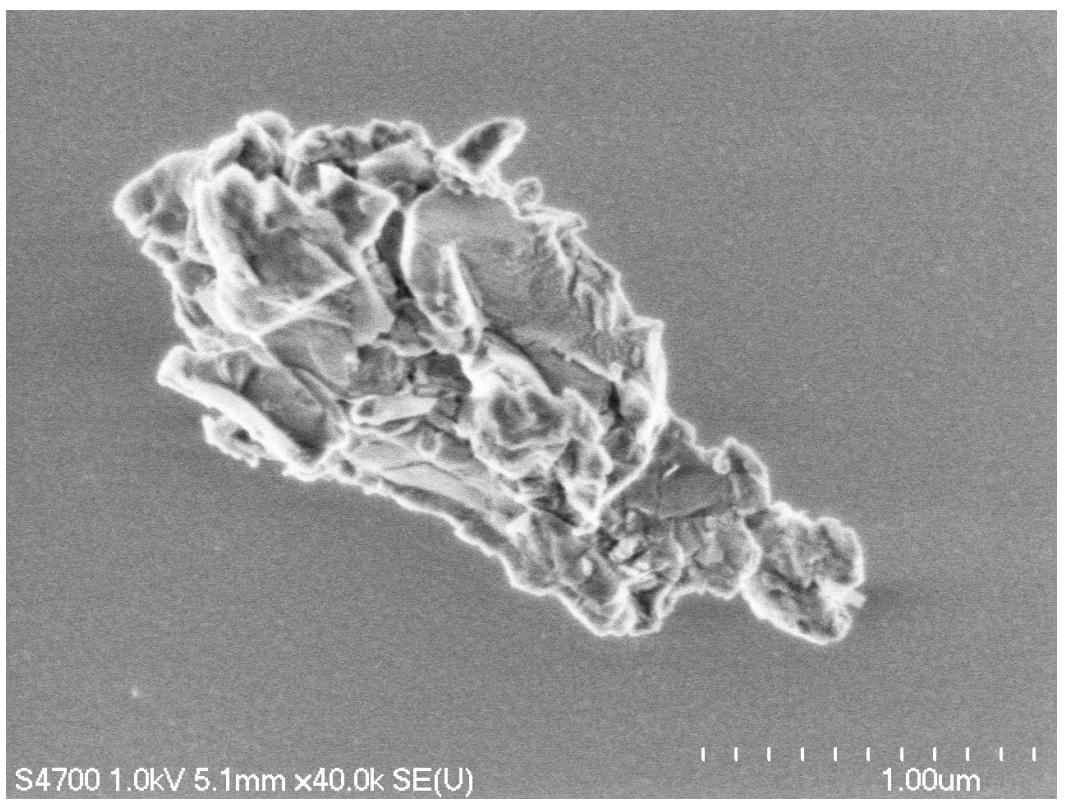

Figure 1.7: A scanning electron microscope (SEM) image of a mineral dust particle. Note the fine scale surface texture and sharp features. Many small mineral dust particles are transported into the troposphere, where they can interact with supercooled cloud water droplets.

the liquid phase ${ }^{2}$. This highlights the need to examine the role of the droplet triple line in our experiments.

We will show in Chapter 3 that the existence of a triple line alone is not a sufficient condition to induce surface crystallization. That experiment utilizes a macroscopic contact line $(r \sim 1 \mathrm{~cm})$, on an atomically smooth substrate. This simple geometry is very different from what we would expect for particle-droplet interactions with "rough" aerosol particles (Figure 1.7). The question then becomes: At what length scale does surface texture becomes important for nucleation?

\footnotetext{
${ }^{2}$ The conditions required to form microscopic contact lines will be discussed in Chapter 6
} 


\section{Macroscopic vs. Microscopic Contact Lines}

In the experiments described in Chapter 5, three length scales are important; (i) the droplet size $r_{\text {drop }}$, (ii) the size of the surface features $r_{\text {roughness }}$ and (iii) the size of the critical nucleus $r_{\text {nucleus. }}$. It has previously been demonstrated in computational models that as the size of the surface features approaches the length scale of the surface features $\left(r_{\text {roughness }} \approx r_{\text {nucleus }}\right)$, an enhancement in the nucleation rate is observed $[58,77]$. Our observations suggests that for surface features in the vicinity of the droplet triple line (Figure 1.8), surface nucleation is also enhanced when under the same conditions.

For the line tension contribution to be significant it must scale as $\delta \propto \frac{\tau}{\sigma}$, which we calculate to be $\delta<100 \mathrm{~nm}$. The only length scale in the droplet before nucleation is that of the surface roughness, or surface feature size. This suggests that surface features of the appropriate size for heterogeneous nucleation enhancement, $r_{\text {roughness }} \approx r_{\text {nucleus }}$, likely also play a role in an enhancement of surface freezing in the heterogeneous mode when $\delta \approx r_{\text {roughness }}$ if they exist in the vicinity of the droplet's macroscopic triple line. De Gennes [14] suggests that pinning of the macroscopic droplet triple line by these surface features (Figure 1.8) may form a new microscopic contact line with a radius of curvature $r_{\text {TripleLine }} \approx r_{\text {SurfaceFeature }}$

An analog of this might be found for droplets resting on a smooth surface $\left(r_{\text {roughness }}<\right.$ $\left.r_{\text {nucleus }}\right)$. In this geometry, the droplet's triple line would need to be of the order of $\delta$ $\left(r_{\text {drop }}<\delta\right)$. Similarly, for the homogenous case, this could be thought of as making the radius of curvature of the droplet of similar order to $\delta\left(r_{d r o p} \approx \delta\right)$, under which case we 


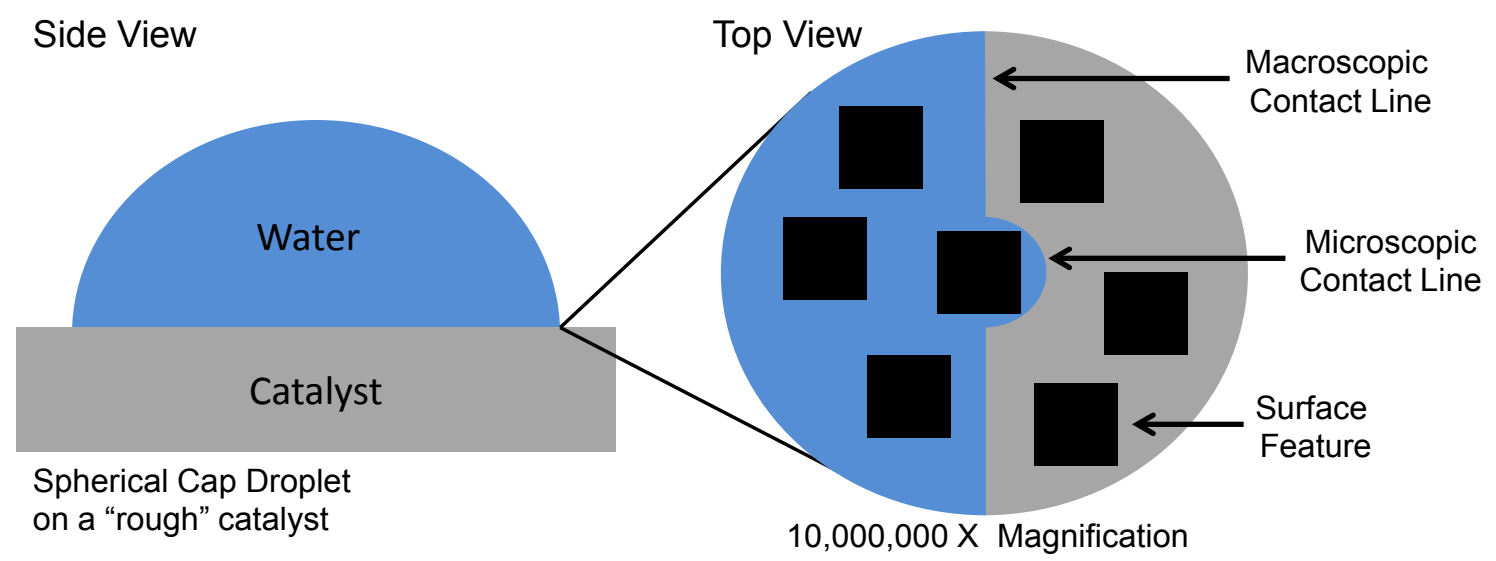

Figure 1.8: Macroscopic vs. Microscopic Contact Line, Left: A spherical droplet rests on a catalyst substrate in a similar geometry to that of Gurganus et al.[31, 32] Right: An enhancement of the region in the vicinity of the droplet contact line, highlights the small surface features (black squares) on the "rough" catalyst. When these features are present at the air-water interface, the macroscopic droplet triple line $\left(r_{\tau \text { Macro }}=r_{\text {Drop }}\right)$ is distorted. A new "microscopic triple line" is created with a radius of curvature based on the size of the feature $\left(r_{\tau \text { Micro }} \approx r_{\text {SurfaceFeature }}\right)$. The new "microscopic" triple line may be sufficiently small $\left(r_{\text {TripleLine }} \sim \boldsymbol{\delta}\right)$ that line tension tension contribution $\left(\sigma_{\tau}=\frac{\tau}{r_{\text {TripleLine }}}\right)$ is may increase the probability of surface freezing $\left(J_{\text {Rough }}>J_{\text {Smooth }}\right)$.

would expect to see the surface nucleation rate dominate nucleation $J_{\text {Surface }} \gg J_{\text {Volume }}$. Both of these scenarios would require formation of droplets volumes of the size $r_{\text {nucleus }}^{3}$, in the range of zepto to yocto liters $\left(10^{-21} L-10^{-24} L\right)$ which are obviously not practical to generate, or applicable to many systems. Because surface features of the necessary size, $r_{\text {roughness }} \approx r_{\text {nucleus }}$, are found on many surfaces it is possible that surface nucleation is only possible in the heterogeneous mode. 


\subsubsection{Surface Texture}

The last contact nucleation pathway is perhaps the most promising for atmospheric nucleation. The surface texture or "roughness" of a surface can change the contact angle of a macroscopic droplet [14], but for an ice crystal that may be smaller than 10nm (Figure 1.3) the role of surface texture on larger scales becomes nebulous. At these small scales, a macroscopically rough surface would appear to be smooth. Nucleation experiments have noted a preference for nucleation to begin at certain "active sites" (scratches, defects, etc.)[38], but in the case of inside-out nucleation, where a fully immersed particle comes into contact with the droplet interface (Figure 1.4 D)[63], these features persist on the surface of the immersed particle and yet nucleation still prefers to begin at the three-phase interface. What is different about rough surfaces when they come into contact with a air-water interface?

A possible answer to that question is found with the inclusion of line tension. Line tension requires the existence of a three phase contact line, which exists permanently at particle-water-air interface. It is true that an particle-ice-water interface exists once the nucleus has formed, but its existence is tenuous before the Gibbs barrier $\left(\Delta G^{*}\right)$ has been crossed. If one of the sharp sites impinges on the droplet interface, in essence "poking the droplet," a small volume of water may form a spherical cap (Figure 1.9). If this spherical cap is sufficiently small, but greater than the size of the critical nucleus $\left(r^{*}\right)$, the formation of the three-phase contact line may act to lower the barrier to nucleation $\Delta G^{*}$ (Figure 1.3). The relevance of this "poking" is uncertain, but it requires the existence of very small features on the aerosol and a very particular orientation of the aerosol. 


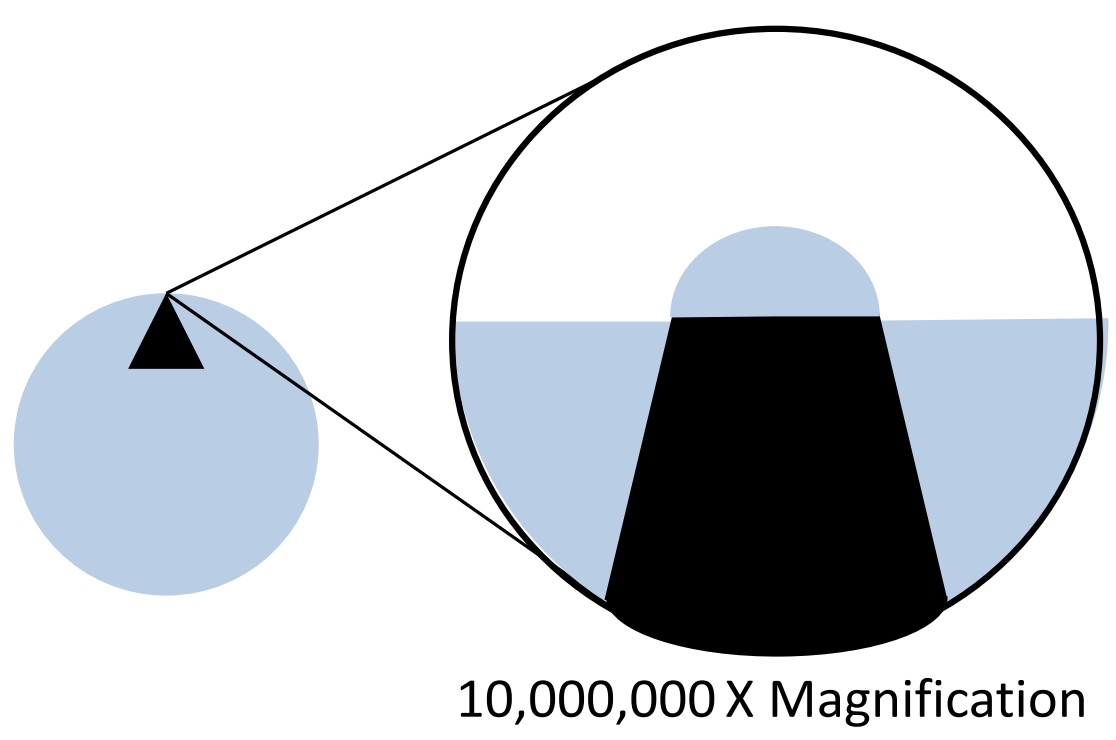

Figure 1.9: A possible mechanism for point-like contact freezing A sharp aerosol extruding from the droplet (Figure 1.4 D), may form a spherical cap of supercooled water at the point of contact. Under certain conditions, the line tension contribution may be significant $r^{*}>r_{\text {poking }} \sim \delta$ (Equation 1.27) leading to a contact mode enhancement.

The "poking" geometry detailed above may not be the only scenario where line tension is important for contact nucleation, indeed this geometry is very difficult to achieve in the laboratory (section 6.5.1). It may be possible for the macroscopic contact line surrounding a partially immersed aerosol particle (Figure 1.4 B) to be stretched by pinning of the receding contact line [14] around a surface site of approximately the same size as the critical nucleus (Figure 1.8). In this scenario, a bit of fluid trapped in the vicinity of the surface site will experience a strong contribution from line tension due the surface area to contact line ratio. Therefore a particle impinging on the surface might not freeze immediately, as is commonly believed for contact nucleation, but may wait for evaporation or mechanical disturbance to shift the macroscopic contact line, resulting in the formation of a microscopic contact line via pinning. 
Both of the poking and pinning scenarios assume that the catalyst particle is smooth, on the length scales necessary for the formation of a critical nucleus. However, there are situations where an aerosol may be porous. Preferential nucleation can begin within small immersed pores[58], even without the contribution of line tension. Therefore there may also be a contribution to contact freezing when pores exist in the vicinity of the air-water interface. 



\section{Chapter 2}

\section{Experimental Methods}

\subsection{Research Outline}

This dissertation is organized around a group of three experiments to better understand the onset conditions for nucleation at the contact line, i.e. heterogeneous surface crystallization. The preceding chapter provided a general overview of nucleation theory and highlighted several mechanisms that could possibly be responsible for the observations of nucleation at the contact line. In the following chapters we will discuss the results of testing three of these hypotheses; Chapter 3: The role of a macroscopic three phase interface, Chapter 4: The role of thermal gradients, Chapter 5: The role of surface texture.

The contents of chapters 3-5 are taken from three articles published during my graduate work. Each was written with a brief summary of experimental methods and theory in addition to the experimental results. In this chapter we will elaborate on the methods and 
equipment used in these three studies.

\subsection{Characterizing Droplet Nucleation}

In these studies droplets were cooled under atmospherically relevant conditions to study nucleation of cloud droplets. To better control the thermodynamic variables, thermal chambers are used to isolate the droplets in the laboratory. In addition to monitoring freezing temperatures, we also introduce a novel technique to the identify the spatial origin of the nucleus. While the cooling technique is altered slightly for each experiment, all three studies utilize the same high speed imaging technique to trace the spatial distribution of freezing sites with high resolution at the catalyst-water interface. To our knowledge, this is the first time that both the distribution of freezing temperatures and the spatial distribution of nucleation sites has been recorded simultaneously in this manner.

\subsubsection{Geometry}

\section{Catalyst: Particle vs. Substrate}

All of the experiments described here rely on a spherical cap droplet resting on a substrate. In this simple geometry, the substrate acts as the nucleation catalyst, which can be thought of as a giant aerosol particle in contact with a droplet. We selected this geometry because it offers a well defined three-phase contact line around the perimeter of the droplet, and a disk shaped two phase immersed region. These regions are assumed to 
be analogous to those formed when an aerosol particle comes into contact with a droplet interface (Figure 2.1).

For this work we have selected prime grade silicon wafers as catalyst substrates due to their uniform surface profiles and chemical homogeneity. All of our wafers were single side polished with RMS roughness values $<1 \mathrm{~nm}^{1}$. The polished wafer is highly reflective (in the visible regime) which is advantageous for our imaging technique. As part of our first study we determined that $\mathrm{p}$ - (boron) and n- (phosphorus) chemical doping of a wafer does not significantly affect the droplet freezing temperature. It is important to note that because our wafers are exposed to air and water, we expected some thin native silicon oxide to form on the surface $\left(h_{\text {oxide }}<1 \mu m\right)$ [24]. The presence of this oxide layer changes the chemical properties of the surface allowing for increased wetting by water $\left(\theta_{\mathrm{Si}-\text { Water }}>\theta_{\mathrm{SiO}_{2}-\text { Water }}\right)$ because pure silicon is more hydrophobic than silicon dioxide[81]. Additionally, irregular growth of this amorphous oxide layer may alter the topology of the substrate, but results from our studies (Chapters 3 and 4) suggests that this does not changes the nucleation properties of the catalyst substrates.

\section{Nucleation Site Location: Immersion Mode vs Contact Mode}

Two of the experiments described here (Chapter 3 and Chapter 4), rely on a substrate to act as the sole nucleation catalyst. When a droplet rests on a substrate, it forms a spherical cap. Because our freezing temperatures are always higher than the homogeneous freezing limit $\left(T_{\text {freeze }}>235 \mathrm{~K}\right)$ required for volume nucleation, we assume that nucleation will be confined to the substrate-liquid interface. In this droplet geometry the interface will be

\footnotetext{
${ }^{1}$ Special thanks to Dr. Drelich at MTU for his assistance with AFM charactarization of our silicon substrates.
} 


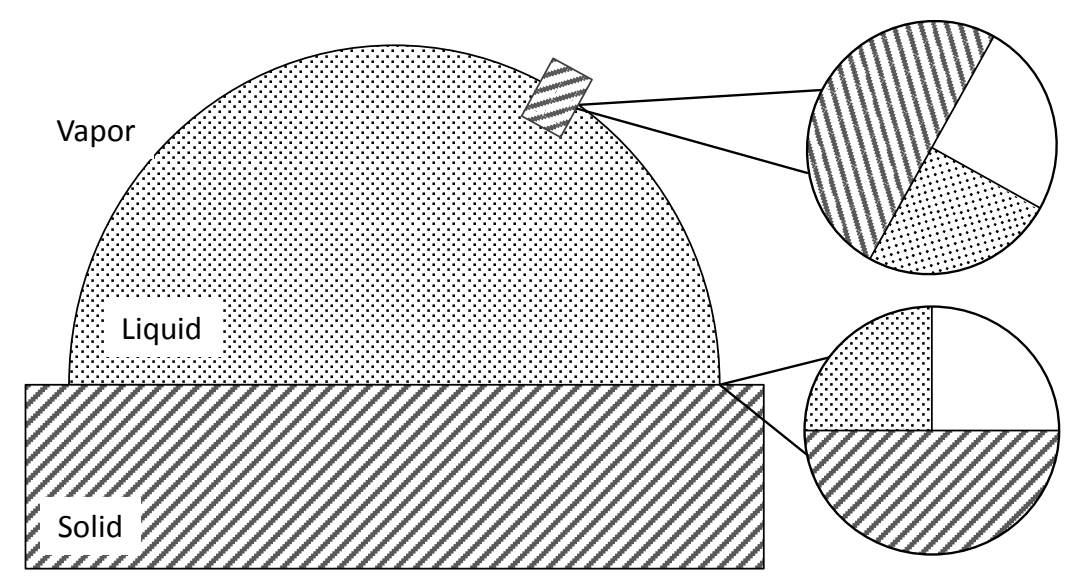

Figure 2.1: Contact Line Similarity The contact line formed at the spherical-cap droplet perimeter is analogous to that one formed at the three-phase (solid-liquid-vapor) interface between an aerosol in contact with a droplet.

circular, allowing for a simple distinction between the circular-two phase immersed region $\left(a_{\text {immersed }}=\pi r^{2}\right)$ and the three-phase contact line at the perimeter $\left(l_{\text {perimeter }}=2 \pi r\right)$. A nucleation site located in the two-phase and three-phase regions will be called immersion mode and contact mode freezing events respectively (Figure 2.2).

As part of the image analysis to identify freezing sites, we are careful to catalog the location for sequential freezes. If two freezes occur sequentially in the same location, then we classify the nucleation site as a defect. Because the distribution of freezing sites is assumed to be stochastic, the probability of sequential and identical freezing sites is exceedingly small. These defect sites are therefore not included in the overall spatial distribution of freezing sites we report. Unfortunately these defect sites account for much of our data, requiring analysis of large numbers of drops to achieve a sufficient data set. These defects could result from outside particles settling on the substrate or some other mechanism, but it is curious to note that the majority of them appear on the droplet 


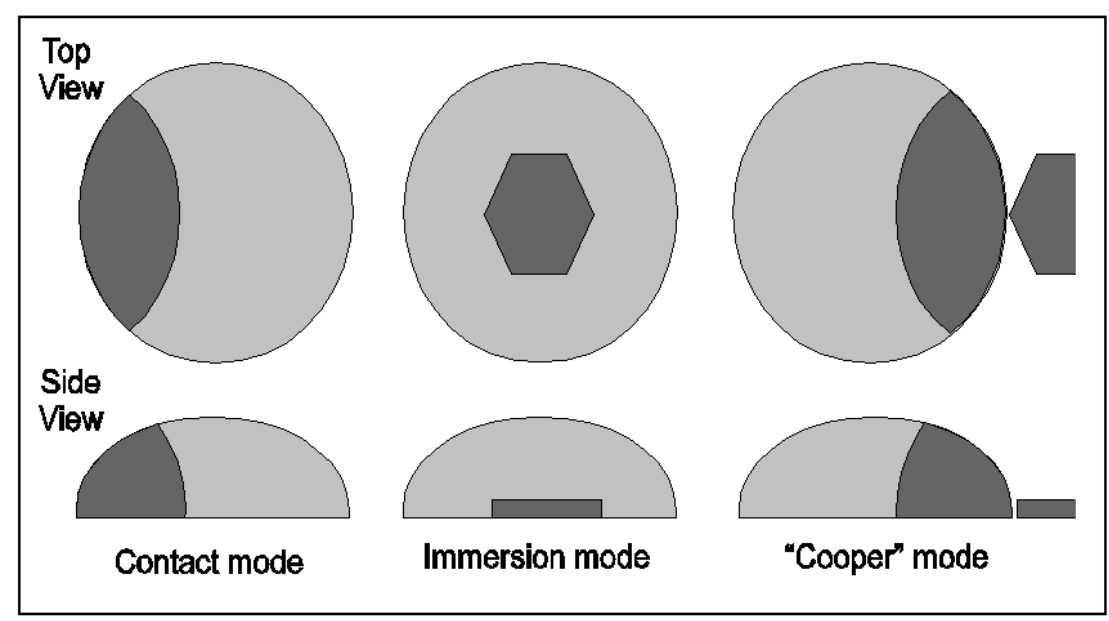

Figure 2.2: Contact vs. Immersion Nucleation of a Spherical Cap Droplet A schematic top and side view of initial crystallization of the drop (dark grey). Contact mode nucleation will begin at the droplet three-phase (substrate-water-air) perimeter line . Immersion mode nucelation will begin in the two-phase substrate-water interracial area. A third scenario where a crystal growing external to the droplet triggers nucleation, in a similar fashion to that described by Cooper [13] is occasionally observed for some substrates, but these instances are filtered from that data and will not be considered here.

perimeter. It is important to note that in these experiments we utilize a filtered air or nitrogen supply to minimize aerosol contamination in the chamber.

To minimize any possible bias, the nucleation substrates were selected to be as smooth as possible. We selected silicon wafers as substrates for their atomically smooth surfaces, homogenous chemistry, low cost and reflectivity (a requirement with our vertical camera and lighting configuration). These wafers are hydrophillic $\left(\theta_{\text {contact }}<60^{\circ}\right)$, but due to pinning of the contact line the contact angle was observed to decrease as the droplet evaporated with repeated freeze-thaw cycles. In our second study (Chapter 4) we also treated a subset of wafers with a commercial silane treatment (RainX) to increase the hydrophobicicity of the substrate $\left(\theta_{\text {contact }} \approx 90^{\circ}\right)$. With all wafers, a small (typically 
white) mineral residue remained on the wafer after the droplet evaporated completely. We attribute this to chemical impurities in our water supply, but do not believe that it biased our observations because of our filtering techniques during post processing of our spatial data. In our final study the substrate does not act as the primary catalyst so we introduced commercial available hydrophobic "siliconized" glass wafers from Hampton with similar contact angle to the RainX treated wafers.

\subsubsection{Freezing Characteristics of Supercooled Droplets}

A supercooled droplet will typically freeze in two stages, the first defined by rapid crystallization of the droplet interfaces and a second slower one in which the interior volume of the droplet undergoes a phase change [3]. Stage one occurs in a fraction of a second, due to the presence of supercooled water and the efficiency of heat transfer at the drop surface. Stage two can last many seconds and is limited by the need to remove excess latent heat. The second stage begins as the droplet temperature is warmed to the melt temperature, where heat must be dissipated into the environment surrounding the droplet. By continually monitoring the temperature of the droplet we can use this property to identify a freezing event by a sudden spike in the freezing temperature (Figure 2.3).

As the droplet freezes it becomes opaque, a property we exploit to identify freezing. This scattering of light is caused by the trapping of tiny bubbles in the rapidly forming ice

lattice. A fraction of the incident light transmitted $\left(T_{I}=\frac{I_{\text {in }}}{I_{\text {out }}}\right)$ is governed by Fresnel's law 


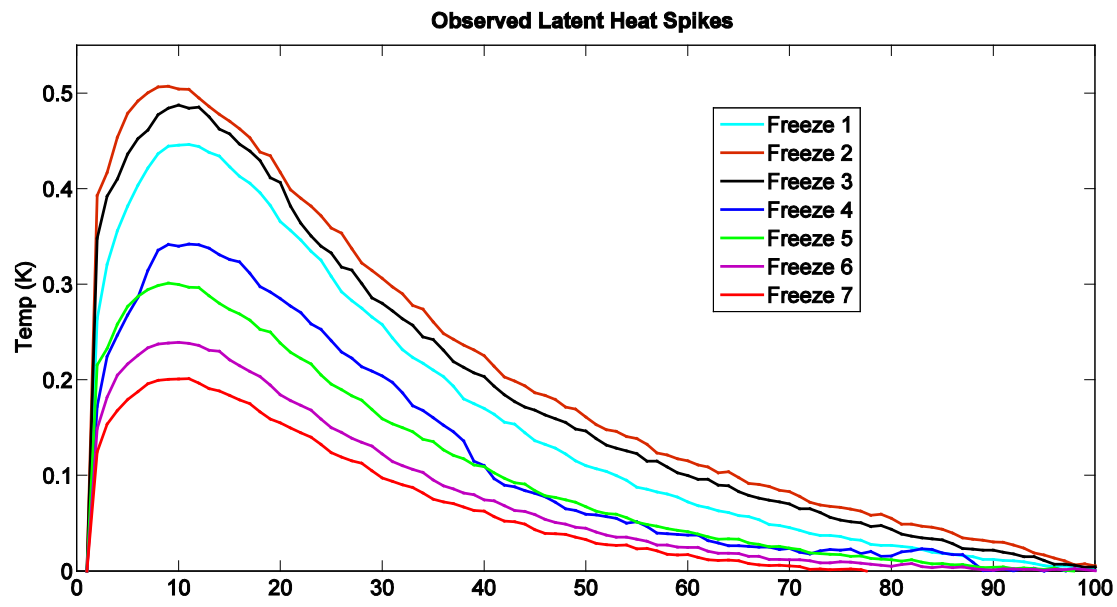

Figure 2.3: Latent Heat Release Warming of the air in the vicinity of a freezing droplet due to latent heat release. The size of the heat spike (subtracting the ambient temperature) is proportional to the volume of the droplet, with the decrease here indicating an evaporating droplet over multiple freeze-that cycles. These air temperature profiles were recorded during the experiments described in Chapter 3[31]

(assuming the idealized case a planar interface and normal incidence $\theta_{i}=0^{\circ}$ )[33]

$$
T_{I}=\frac{4 n_{1} n_{2}}{\left(n_{1}+n_{2}\right)^{2}}
$$

with $n_{1}$ and $n_{2}$ being the index of fraction of ice and air respectively. In this case $\left(n_{\text {ice }}=1.3\right.$, $n_{\text {air }}=1$ ) each bubble will scatter $\approx 2 \%$ of the incident light. The vast amount of bubbles trapped in the ice lattice aggravate this effect, causing the frozen droplet to appear opaque $\left(T_{I} \ll 1\right)$.

\subsubsection{Imaging}

In these studies, the droplet and substrate are continuously cooled until a freezing event occurs. To identify the nucleation site within the droplet during the rapid first 
stage of freezing, we employ high speed imaging $(5-10 \mathrm{kHz})$. A vertically pointing high speed camera with a macro lens looks down on the droplet-substrate interface, continually recording during the cooling stage through the use of a ring buffer. A trigger during freezing stops the recording and the buffer is then saved to disk. After the cooling runs are complete, each video is compressed and analyzed with ImageJ software. With this software we can identity the area of the droplet-substrate interface and, by replaying the film backwards, the initial nucleation site (Figure 2.4).

\subsection{Isothermal Chamber (Chapter 3)}

In our first study, described in chapter 3[31], we sought to prevent any thermal gradients though the use of an isothermal environment which closely resembles static atmospheric conditions. This was accomplished with a small chamber with a copper lined volume that was cooled by the flow of antifreeze through channels around the exterior of the volume (Figure 2.5). O-rings sealed around the fluid channels to prevent coolant from leaking into the volume. The top of the chamber could be removed to place a substrate and droplet on a platform at the bottom. A small plastic structure was designed to hang from the top panel to position LEDs, an RTD and a photodiode above the droplet. A port on the center axis of the panel was designed to hold a 1 diameter inch window. Smaller ports above the superstructure were used for electrical connections and airflow into the chamber.

\subsection{Thermal Chamber (Chapter 4-5)}

Copper was selected for its high thermal conductivity in order to maintain a homogeneous temperature in the chamber. Given the size and mass of the chamber and 


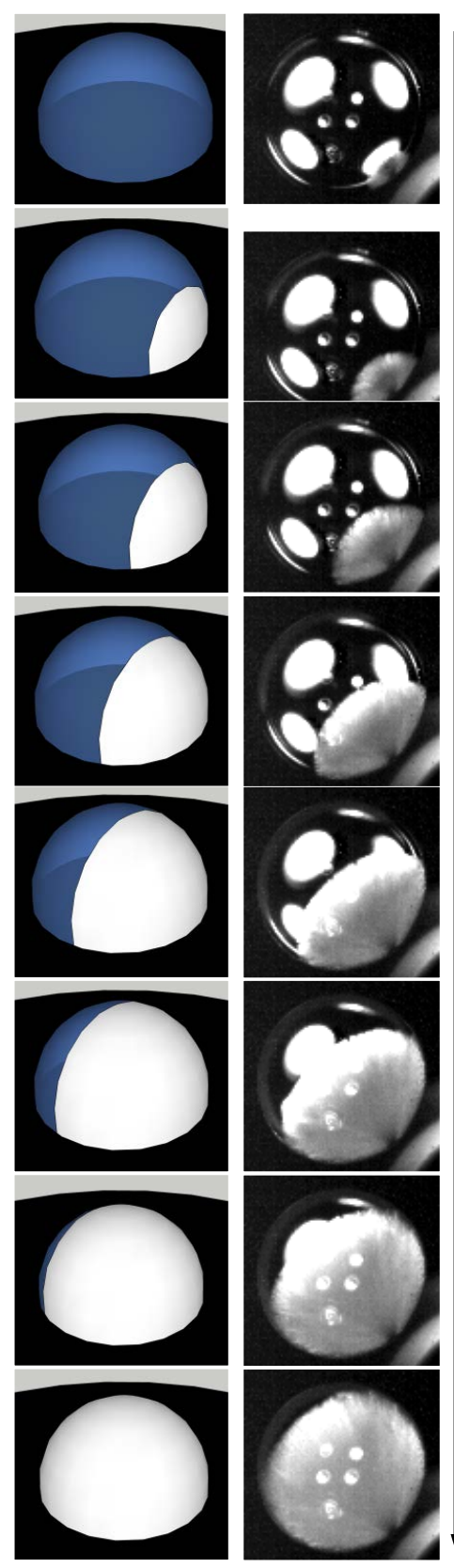

Contact Mode
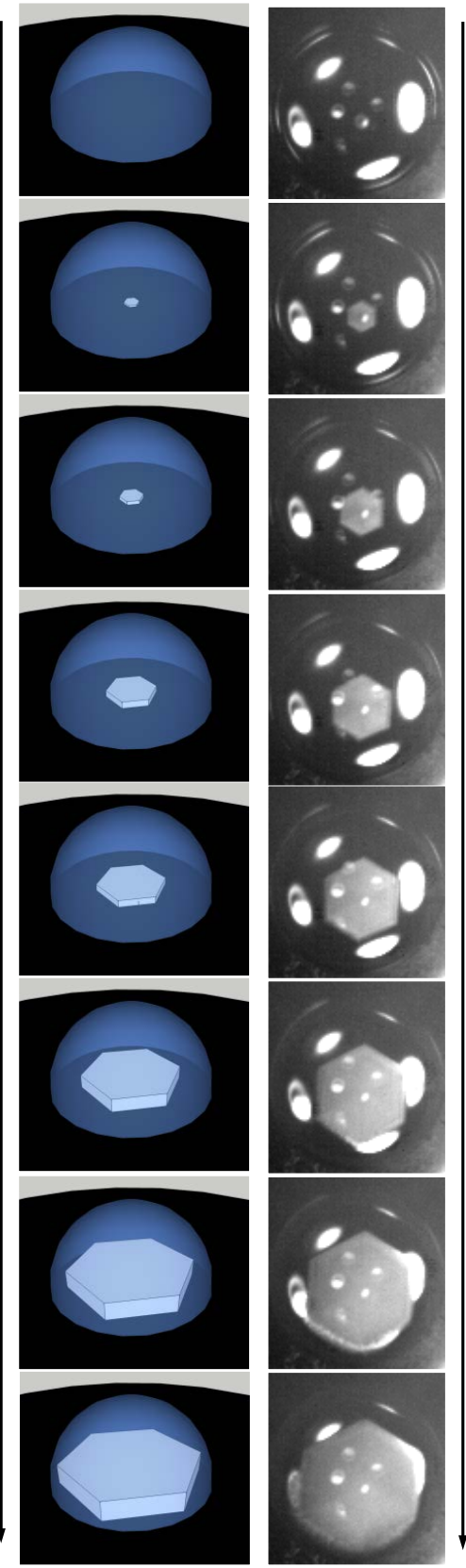

Immersion Mode

Figure 2.4: Identifying the Nucleation Site The nucleation site can be clearly identified by reviewing the high speed nucleation video through the window on top of the thermal chamber. Here, every 5 th frame of a $5 \mathrm{kHz}$ video is shown for a contact mode nucleation event beginning at the three phase interface on the droplet perimeter (left), and an immersion event beginning on the substrate in the two phase region (right). A cartoon for each scenario depicts the spread of the crystal front. 


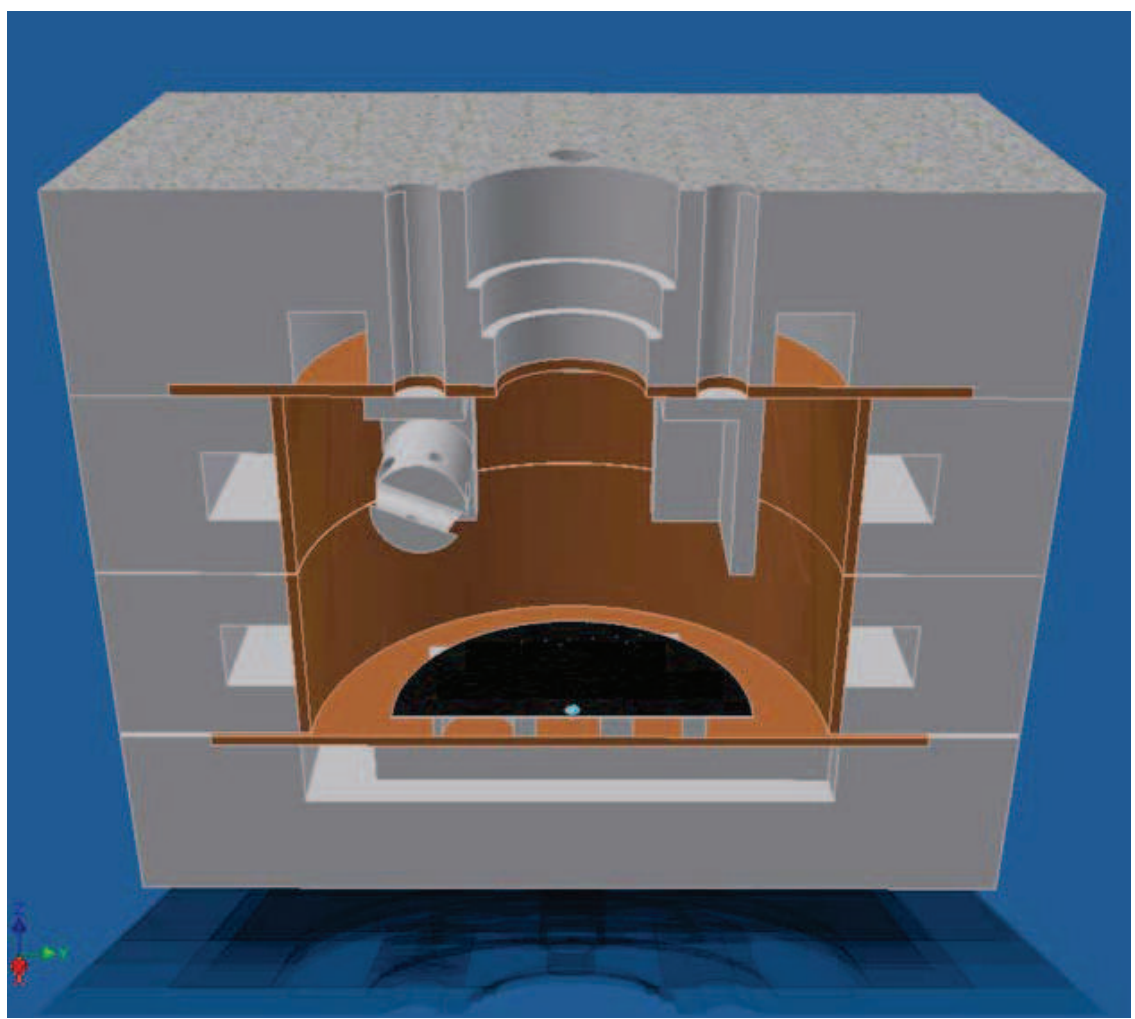

Figure 2.5: A half cut schematic view of the 2011 Isothermal Chamber A water droplet (blue) resting on a silicon wafer (black) sits on a small stand in the middle of the copper lined chamber volume. The chamber body is machined in 4 pieces from Acetal polymer (grey), with channels milled for antifreeze to flow around the copper walls. A viewing window (not shown) located directly above droplet, allows for imaging of the droplet-substrate interface. A plastic measurement package is suspended above the droplet to monitor the volume temperature, and provide off axis illumination.

the choice of circulation cooling, the thermal response time was was long so we were limited slow cooling rates $(\lambda<1 K / \mathrm{min})$. The cooling rate was controlled by setting the temperature of a circulation chiller, through a Labview interface. A jet of air was also directed at the exterior window to prevent frost formation. Overall this chamber fulfilled its role in minimize thermal gradients, but the very slow response time of the circulation cooling led us to redesign the system for later tests. 
In our second experiment, we sought to rapidly cool the droplet-substrate system to study the role of thermal gradients. A larger isothermal chamber was designed that allowed for horizontal and vertical imaging of the droplet (Figure 2.6). Like the previous design the copper walls were again cooled by circulation of antifreeze, but an un-cooled plastic middle section (lighter grey) with four optical ports was also added. This new section provides an internal superstructure which supports a copper heat sink for a ceramic solid state peltier cooler (not shown). The peltier cooler is separated from the nucleation substrate by a $1 / 4$ inch copper plate (to damp out any thermal variations). Unlike the previous chamber the circulation chiller maintains the walls and heat sink at a constant temperature during tests $\left(T_{\text {chiller }}=-30^{\circ} \mathrm{C}\right)$.

\subsection{Probing the droplet (Chapter 5)}

\subsubsection{Poking Apparatus}

In our third paper we sought to move away from catalyst substrates, toward a point like contact similar to the concept described by Grossier et al. [29]. To position a probe in a spherical cap droplet, we designed an apparatus to extend a 1/4 inch plastic tube 4 inches to the center of the thermal chamber (Figure 2.7). The probes are placed into the hollow section and can be positioned at the droplet interface with a three axis mechanical stage. Once in position, a piezoelectric line stage attached to the plastic tube allowed us to drive the probe into droplet with $50 \mathrm{~nm}$ precision. A new high-magnification $(5 \mathrm{X})$ camera with a $105 \mathrm{~mm}$ working distance is positioned at one of the perpendicular optical ports to aid in alignment. 


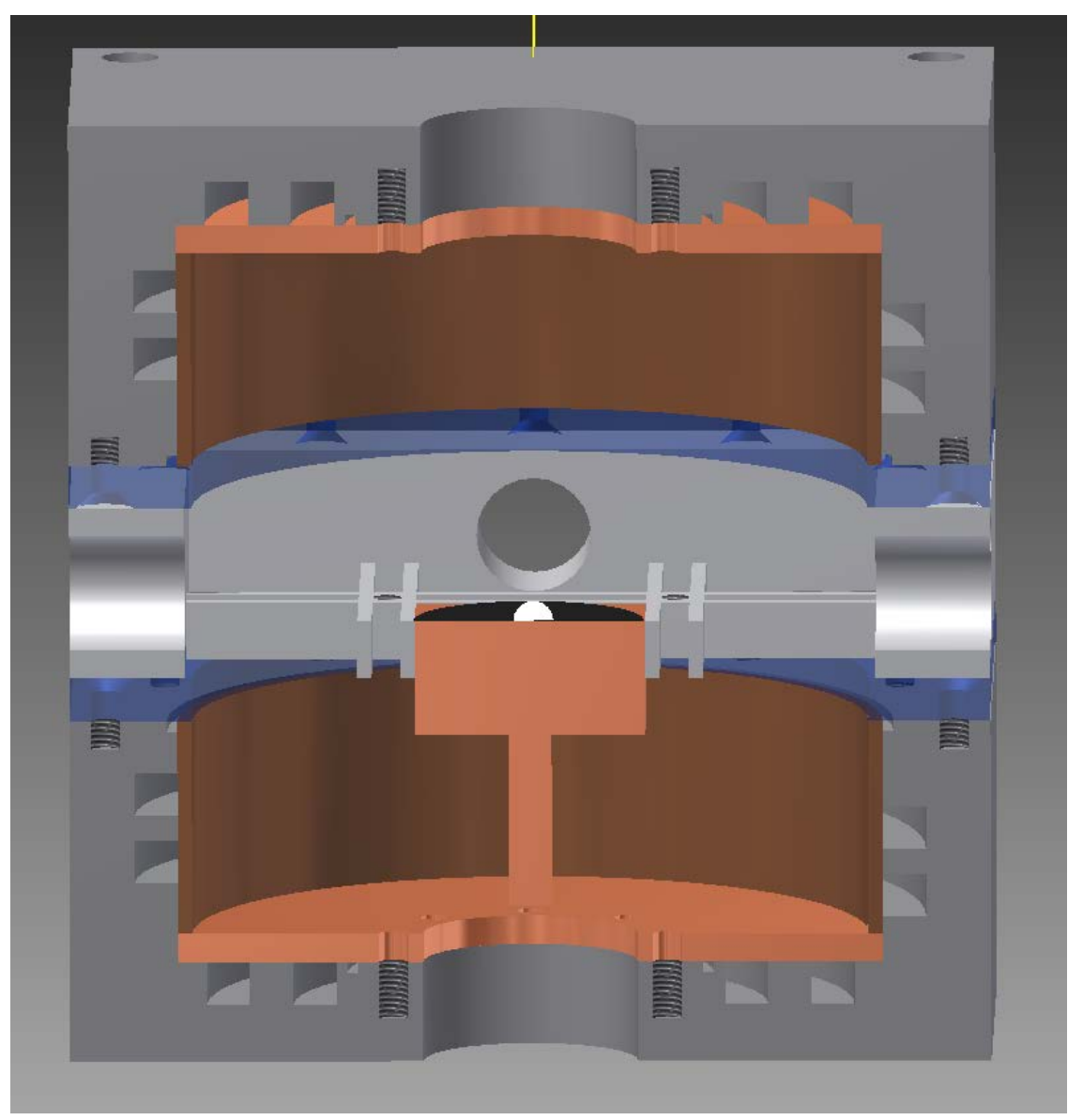

Figure 2.6: A half cut schematic view of the 2013 Isothermal Chamber A droplet (white) is cooled by controlling the temperature of the substrate (black) through the use of a solid state peltier cooler. The copper jacketed walls and the heat sink below the droplet are cooled by circulation of anti-freeze as in the previous chamber.

\subsubsection{Nano-textured Optical Fibers}

We selected optical fibers as probes due to their small size and similar surface chemistry to that of silicon substrates used in the earlier studies (namely the native silicon oxide on the wafers). For more details on the preparation techniques see section 5.4. Our initial goal was to use the etch technique described by De Gennes and Stockle to produce an atomically sharp meniscus by dipping the fiber core in Hydrofluoric acid (HF) $[14,71]$. Unfortunately 


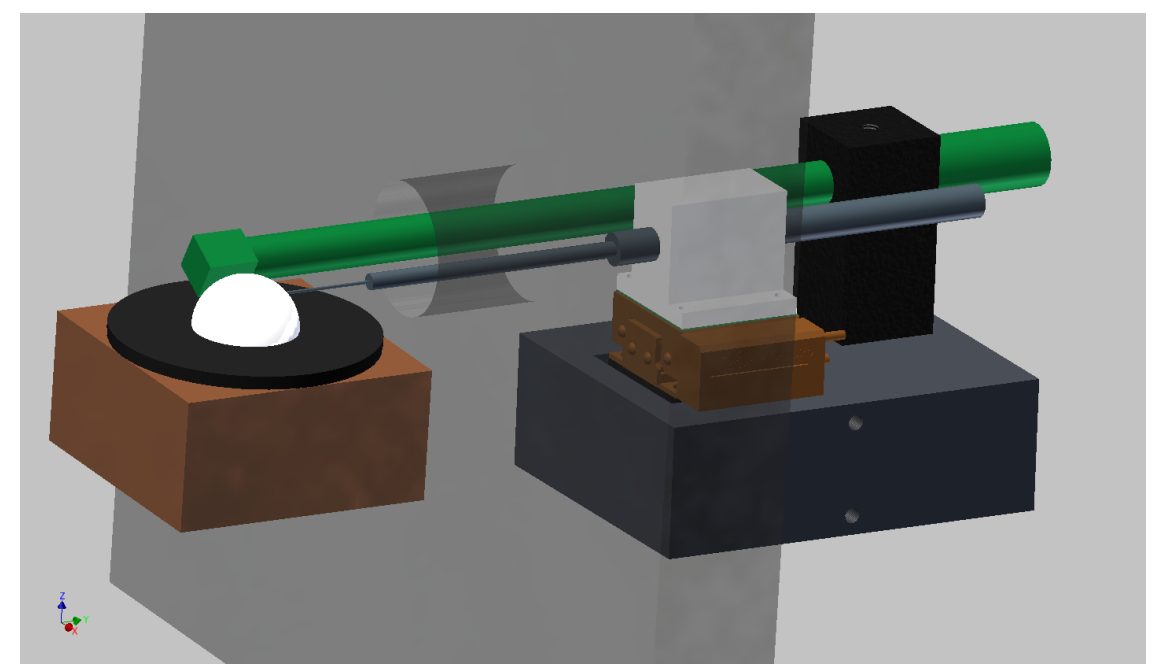

Figure 2.7: The poker fiber is attached to a plastic rod and inserted into the chamber through one of the port holes in horizontal window ports in the thermal chamber. Positioning of the fiber in the droplet is accomplished with a three axis mechanical stage for course adjustments and a piezo electric linear stage for fine adjustments. A right angle endoscope (green) was used for alignment is some situations.

we were never able to achieve the desired sharpness, but we did observe a strange surface texture formation on the silica core (Figure 2.8). The tiny surface features, ranging in size below $r=100 \mathrm{~nm}$, appeared intermittently after stripping of the polymer encasing the silica core of the optical fibers. X-Ray microanalysis of the features indicated that they were composed of silicon and oxygen, identical to the bulk silica fiber. Because this nano-scale texture only occurred on a subset of the fibers, we were able to directly compare the spatial locations of nucleation sites for both "smooth" and "nano-textured" fibers. 

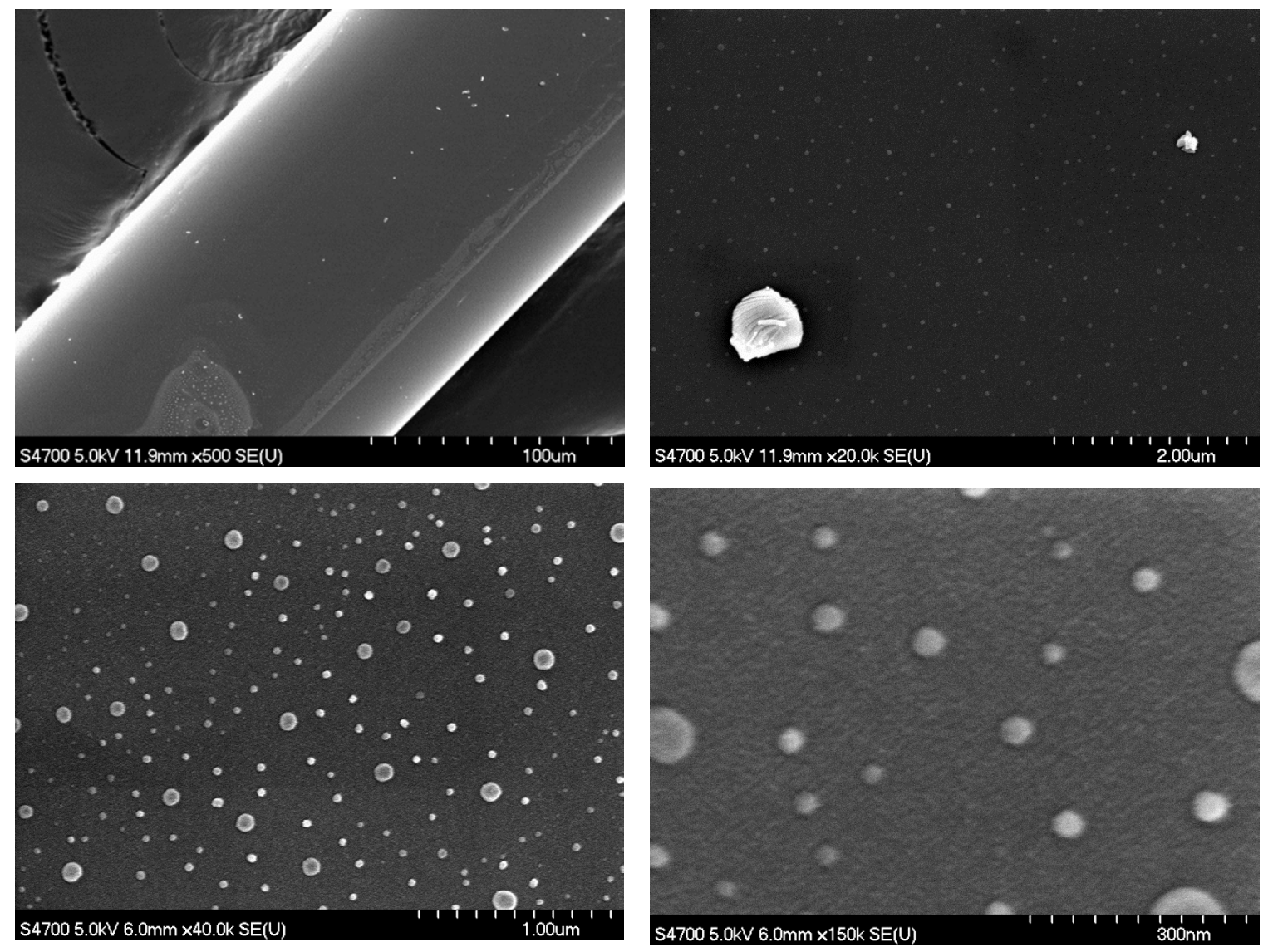

Figure 2.8: Nano-Scale Surface Features on an Silica Fiber A cascade of SEM images for a fiber that has had its plastic cladding removed by etching in a sulfuric acid bath for $\approx$ one hour. At the lowest image resolution (top left), the fiber diameter $(\mathrm{d} \approx 145 \mu \mathrm{m})$ appears to be smooth with a few dust particles deposited on the surface. Zooming in on one of the dust particles (top right) reveals an array of smaller scale surface features. At the highest resolutions (bottom) the features appear circular and have a distribution of sizes down to $10-20 \mathrm{~nm}$. 


\section{Chapter 3}

\section{The Role of a Three Phase Interface}

\section{Fast Imaging of Freezing Drops: No Preference for Nucleation at the Contact Line [31]}

This chapter details a first study into the role of the three phase contact line in heterogeneous nucleation, and was based on a research collaboration ${ }^{1}$. This work is published in full form in the Journal of Physical Chemistry Letters ${ }^{2}$, and is reprinted with permission by the American Chemical Society.

\footnotetext{
${ }^{1}$ This paper is co-authored by A. B. Kostinski \& R. A. Shaw

${ }^{2}$ Reprinted with permission from:

Gurganus, Colin, Kostinski, Alexander B and Shaw, Raymond A, The Journal of Physical Chemistry Letters, 2, 1449-1454, 2011 . Copyright 2011 American Chemical Society.
} 


\subsection{Abstract}

We employ high-speed imaging of supercooled water drops to study the recently reported phenomenon of surface crystallization. Our geometry avoids the "point-like contact" of prior experiments by providing a simple, symmetric contact line (triple line defined by the substrate-liquid-air interface) for a drop resting on a homogeneous silicon substrate. Furthermore, the imaging configuration localizes nucleation sites in the horizontal plane so that their spatial distribution can be examined directly for possible preference near the contact line. Additionally, by using low cooling rates and avoiding substrate cooling, our design minimizes temperature variation within the water drop. The 189 freezing events display nearly perfect spatial uniformity in the immersed (liquidâĂŞsubstrate) region and, thereby, no preference for nucleation at the triple line. This is in contrast to prior experiments where a strong preference for surface freezing (in the contact mode) was observed.

\subsection{Introduction}

Supercooled metastable liquids are ubiquitous but eventually nucleate the thermodynamically stable solid phase. Whether the nucleation occurs with the help of a foreign substance (heterogeneous nucleation) or without it (homogeneous nucleation), uniform probability of cluster formation is typically assumed, scaling with surface area and volume, respectively. Recently, however, the volume scaling for homogeneous nucleation has been questioned in favor of crystallization occurring preferentially at the 
liquid surface (e.g., liquid-vapor interface). If so, homogeneous nucleation rates should scale with surface area rather than volume.[74, 17, 12, 36, 72, 3, 45, 9] Specifically for water, conflicting evidence has mounted regarding the possible preference for surface crystallization.[74, 3, 18] The question is not merely academic as, for example, prediction of crystallization rates in supercooled atmospheric clouds is essential for understanding Earth's radiation balance.[7]

Studies of heterogeneous nucleation also suggest a possible interfacial enhancement. It was recently observed, for example, that particles immersed in a supercooled water drop initiated freezing at temperatures 3-5 $\mathrm{K}$ higher in the vicinity of the air-water interface than in bulk water.[68, 19, 23] The apparently related phenomenon of contact freezing (dry, ice-nucleating particle contacting a supercooled cloud drop) has long been observed to be more efficient than immersion nucleation.[7] These observations stimulated, in part, the theoretical work of Sear,[64] who used a simple Potts model to show that nucleation can favor the contact or triple lines (analogous to the substrate-water-air contact line). Preference for triple line nucleation was also observed in experiments by Suzuki et al.[73] Djikaev and Ruckenstein[16] conjectured that line tension could explain such a preference. In contrast, the observation of disordered layers at the ice-air interface[26] might suggest that interfaces would not be preferred for ice formation. Given the importance of heterogeneous ice nucleation and the paucity of data, we have devised a different approach to study the role of the triple line in heterogeneous nucleation.

Theoretical developments have taken thermodynamic equilibrium for granted, but it should be observed that continuously cooled drops are not in thermal equilibrium. Furthermore, evidence for the enhanced interface contribution to heterogeneous nucleation 
comes from localized, point-like contact between the drop and seed particle, whereas the notion of surface crystallization is more fundamental and need not involve such asymmetric disturbances. Toward a cleaner test of possible contact nucleation and the line tension conjecture, we have developed an experimental approach that (i) avoids asymmetric localized contact, thereby disentangling the notion of line tension from the contact, per se, (ii) addresses the preferential nucleation mode (immersion versus contact) directly in terms of the spatial distribution of nucleation sites rather than in terms of temperature differences, (iii) has a simple symmetric and clearly defined triple line, (iv) decouples the heat sink from the nucleating region, and (v) minimizes temperature variation within the drop. The original motivation came from Sear's[64] simple geometry, water vapor and supercooled water in contact with a uniform nucleation substrate (i.e., constant nucleation rate over the surface). Also, to ensure equal opportunity for nucleation throughout the supercooled water, it is essential to minimize temperature gradients within the drop. Indeed, we note that water density and surface tension, in addition to the nucleation rate, are temperature-dependent. Therefore, it is conceivable that heat fluxes, particularly at point contacts, may influence nucleation. This is why we formulate the contact versus immersion nucleation problem geometrically; is there a statistical preference for nucleation on the triple line in the spatial distribution of nucleation sites? This way of asking the question avoids the notion of nucleation rate and its temperature dependence altogether.

In the present experiment, rather than placing a small seed particle at the surface (i.e., at the liquid-air interface or contact mode) or inside of the droplet (i.e., immersion mode), we let the substrate act as the nucleation medium. The geometry is simple; a supercooled water droplet rests on a uniform, isothermal ice-nucleating substrate, such that initial ice formation can occur either at the liquid water-substrate interface or at the liquid 
water-substrate-air triple line (see Figure 3.1). By selecting a clean homogeneous substrate, we can remove the uncertainties associated with traditional seed particles and provide a spatially uniform probability for freezing. The freezing can be initiated anywhere within the drop-substrate interface, and a high speed movie of the freezing process localizes the nucleation site, as illustrated in Figure 3.1 (right panel). Over repeated cycles of freezing and melting, we determine statistically whether freezing tends to prefer the two-phase interface or the triple line.

Implicit throughout the literature is the notion that drops possess a definite temperature. We observe, however, that this is not so for heterogeneous nucleation experiments, where water drops are cooled at some finite rate $\lambda=\frac{d T}{d t}$. Drops cannot adjust instantly to changes in ambient temperature, but rather, temperature gradients and heat fluxes develop within the drop. Are these negligible, or do they influence nucleation phenomena? To estimate the gradients, note that if an ambient temperature is changed suddenly, and subsequently maintained, the drop approaches a new equilibrium exponentially, with the time constant $\tau=\frac{d^{2}}{\chi}$, where $\chi$ is the thermal diffusivity, $\mathrm{d}$ is the drop diameter, and $\tau$ is known as the thermal relaxation time in the heat transfer literature (approximately $9 \mathrm{~s}$ for a $\mathrm{d}=1 \mathrm{~mm}$ supercooled water drop). In Figure 3.2, we introduce the characteristic thermal variation parameter, $\Delta \mathrm{T} \approx \lambda \tau$ and survey various experiments (including the present one) from this perspective. Because of thermal gradients, the colder portion of the volume will be favored for ice nucleation to some extent. How small should temperature variation within the drop be? On the basis of observations as well as theoretical estimates from classical nucleation theory, we adopt a tolerance of $0.1 \mathrm{~K}$ (see Figure 3.2), corresponding to approximately $10 \%$ variation in the nucleation rate for typical supercooling.[55] 

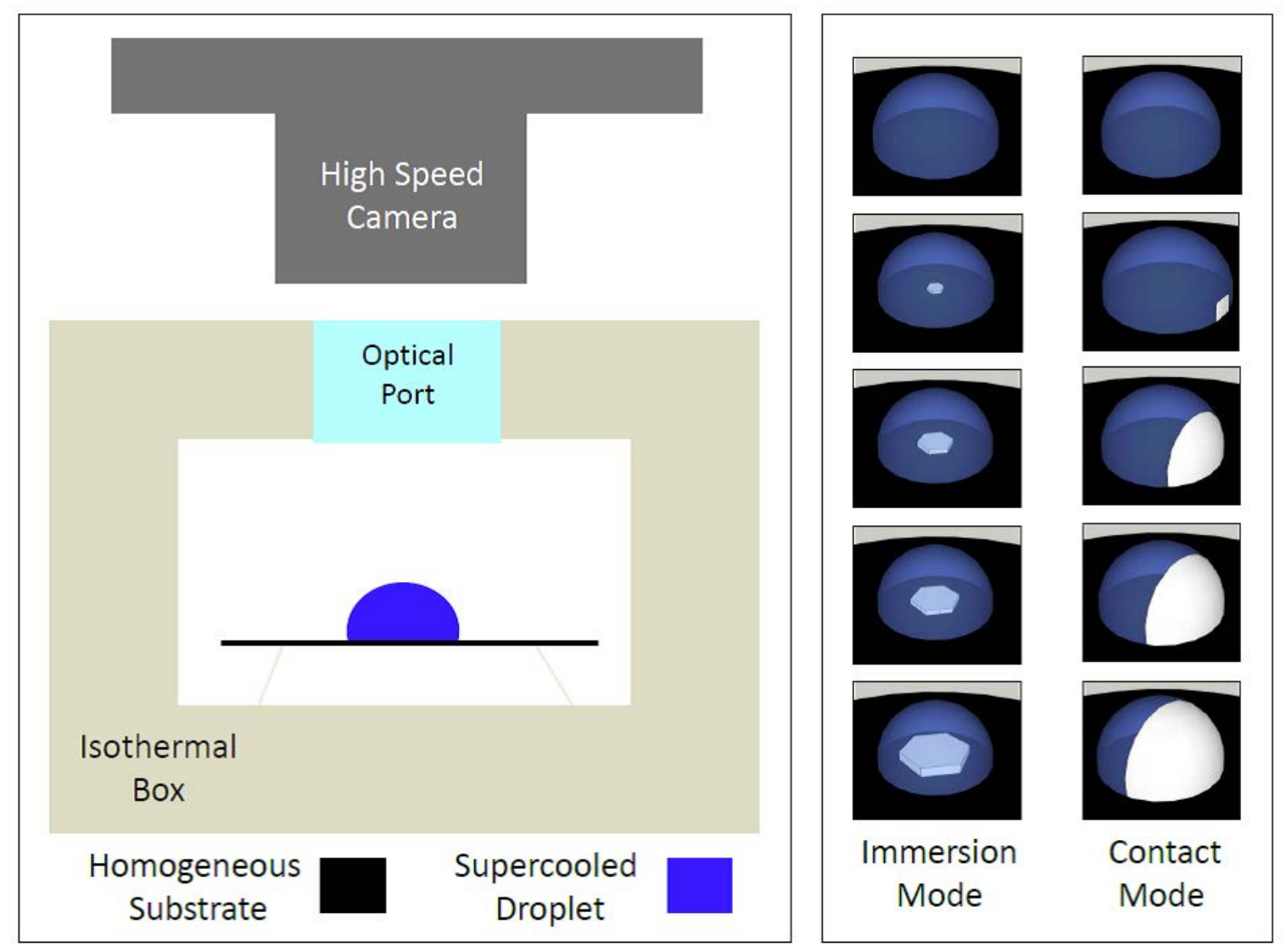

Figure 3.1: Experimental design and nucleation geometry. (Left) Schematic side view of the experimental setup. The high-speed camera is configured to pinpoint locations of freezing events on the substrate. The drop rests on a clean homogeneous and isothermal silicon wafer. The droplet and substrate are encased in an isothermal box, and the walls cool slowly, thereby minimizing temperature gradients. (Right) Schematic perspective view of two freezing drops, illustrating an interior substrate event (immersion mode) and an event occurring at the triple line (contact mode).

To minimize the thermal variation in our study, (i) air itself is used to cool the drop and the substrate inside of an isothermal container, decoupling the nucleating region (the substrate) from the heat sink (see Figure 3.1), and (ii) a low cooling rate is used. Specifically, for our drop sizes $\left(\mathrm{V}=5 \mathrm{~mm}^{3}\right)$ with $\chi \approx 0.1 \mathrm{~mm}^{2} / \mathrm{sec}$, [4] we obtain a characteristic time of $\tau \approx 30 \mathrm{~s}$. Therefore, to maintain a temperature variation of $\Delta \mathrm{T}=0.1$ $\mathrm{K}$, we must limit the cooling rate $\lambda=\Delta \mathrm{T} / \tau$ to approximately $0.2 \mathrm{~K} / \mathrm{min}$. In this regime, the 


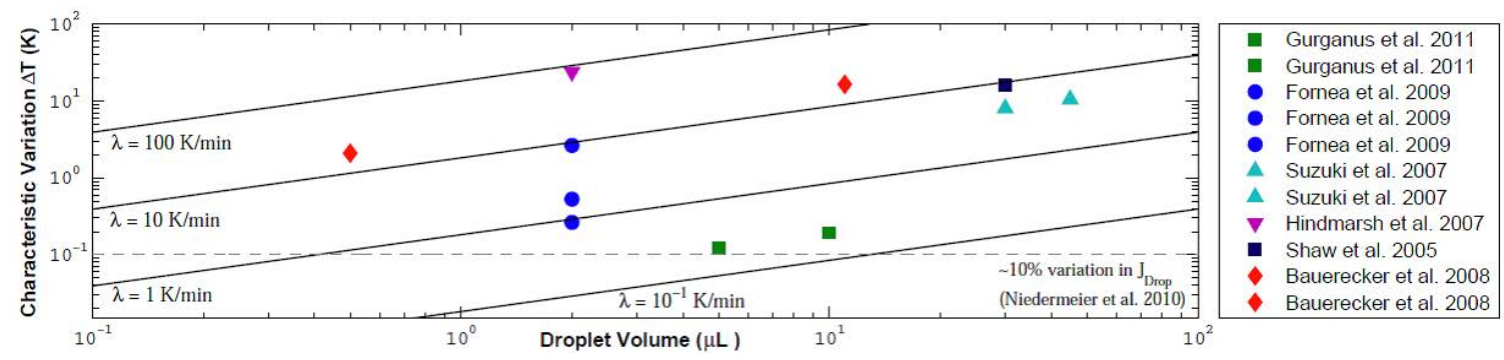

Figure 3.2: Deviation from thermal homogeneity (equilibrium) within drops, subjected to steady cooling. Here, we present likely thermal variations $\Delta \mathrm{T}$ for various experiments, estimated on the basis of thermal relaxation time $\tau$ and applied cooling rate $\lambda$. The thermal relaxation time is estimated using a characteristic length scale $V^{1 / 3}$ (for aspherical drops). Contours of the experimental control parameter $\lambda$ are also shown. Points are shown for the conditions of the homogeneous experiments of Bauerecker[3] (1.5 and $4.5 \mathrm{~mm}$ acoustically levitated drops placed in a chilled volume, with a cooling rate of $20 \mathrm{~K} / \mathrm{min}$ estimated from their Figure 3.3; droplets had varying salt concentrations) and Hindmarsh[36] (spherical droplet suspended from a thermocouple with a cooling rate of $90 \mathrm{~K} / \mathrm{min}$, as estimated from their Figure 3.2; droplets had varying sucrose concentrations), as well as the heterogeneous experiments (spherical cap droplets on an actively cooled substrate) of Fornea[23], Gurganus (presented here), Shaw [19], and Suzuki[73].

spatial temperature distribution within our sample volume can be regarded as quasi-steady, governed by Laplace's equation $\nabla^{2} \mathrm{~T}=0$ with time-dependent boundary conditions. To that end, we use isothermal walls and the maximum (Dirichlet) principle to ensure thermal homogeneity within the entire chamber.

This experiment depends primarily on our ability to determine the location of the initial nucleation site within our sample droplets. In order to achieve a suitably high frame rate (20,000 frames per second), we must subsample the CMOS sensor of our camera to 256 by 256 pixels, resulting in spatial resolution of $250 \mu \mathrm{m}$ (or 5 pixels at $50 \mu \mathrm{m} /$ pixel). It is assumed that ice is formed via heterogeneous nucleation at the substrate. This assumption is reasonable as our freezing temperature limit $\left(T_{\text {Freeze }}>22^{\circ} \mathrm{C}\right)$ is well above 
the homogeneous nucleation threshold of approximately $-35 \mathrm{C}[61]$. As a further test, a right angle mirror was placed near the droplet during several test runs with a $5 \mu \mathrm{L}$ drop. We observed a delay between the nucleation event and the propagation of the freeze front to the top of the droplet. While we were unable to observe the very bottom layer of the droplet with the mirror, these observations rule out nucleation in the upper volume of the droplet.

Examples of sequential frames taken from typical freezing events are shown in Figure 3.3. The initial location of nucleation and the freezing "front" progression are marked by the shift in opacity during the phase transition. The left column shows frames from a movie of a freezing event that initiated, as nearly as we can determine, at the triple line (i.e., analogous to surface crystallization or contact nucleation). The right column shows the progression of a freezing event initiated in the two-phase contact region (i.e., analogous to immersion nucleation).

Over the 189 freezing events, we observed both contact and immersion nucleation events. The distribution of nucleation sites is shown in Figure 3.4 (top panel), and it appears to be uniform, with no obvious preference for the perimeter (the triple line). This can be quantified by sorting events into equal area zones, with the minimum width determined by our spatial resolution (approximately 5\% of drop radius), as shown in the bottom panel of Figure 3.4. For a uniform distribution, we expect Poisson-distributed counts, with 19 events per zone, as shown by the solid horizontal line, and a variance (equal to the mean) as denoted by the dashed horizontal lines. The observed variability is within this range, and most importantly, the 10th bin (corresponding to likely contact nucleation events) is well within the bound. 


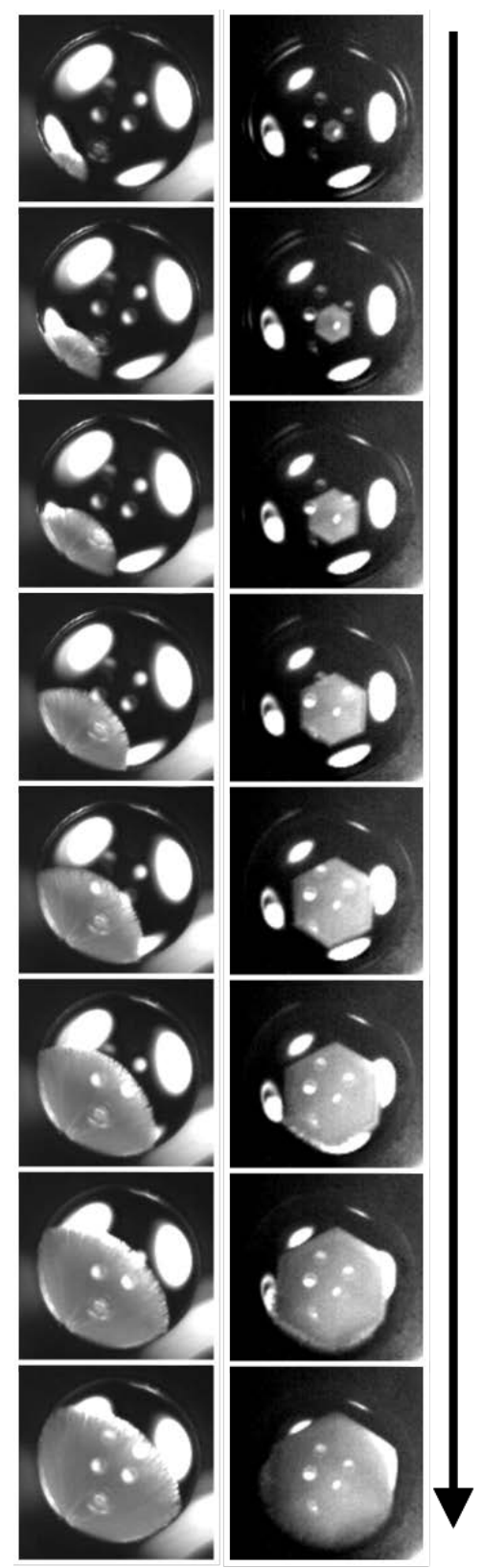

Figure 3.3: Progressions (time evolves downward) of contact (left) and immersion (right) nucleation events for a $10 \mu \mathrm{L}$ spherical cap droplet, viewed from above. The images have been enhanced for contrast, with the freezing front appearing in gray and bright LED reflections evident. In the immersion film, the nucleated crystal is oriented with the basal plane aligned with the imaging plane, thereby showing a hexagonal shape, but this is not necessarily the preferred orientation. These videos were recorded at 20,000 frames per second, with every 20th frame displayed $(0.001 \mathrm{~s}$ time steps between images). A movie of the two freezing events (played at $6 *$ $10^{4}$ times live speed) is available as Supporting Information. 

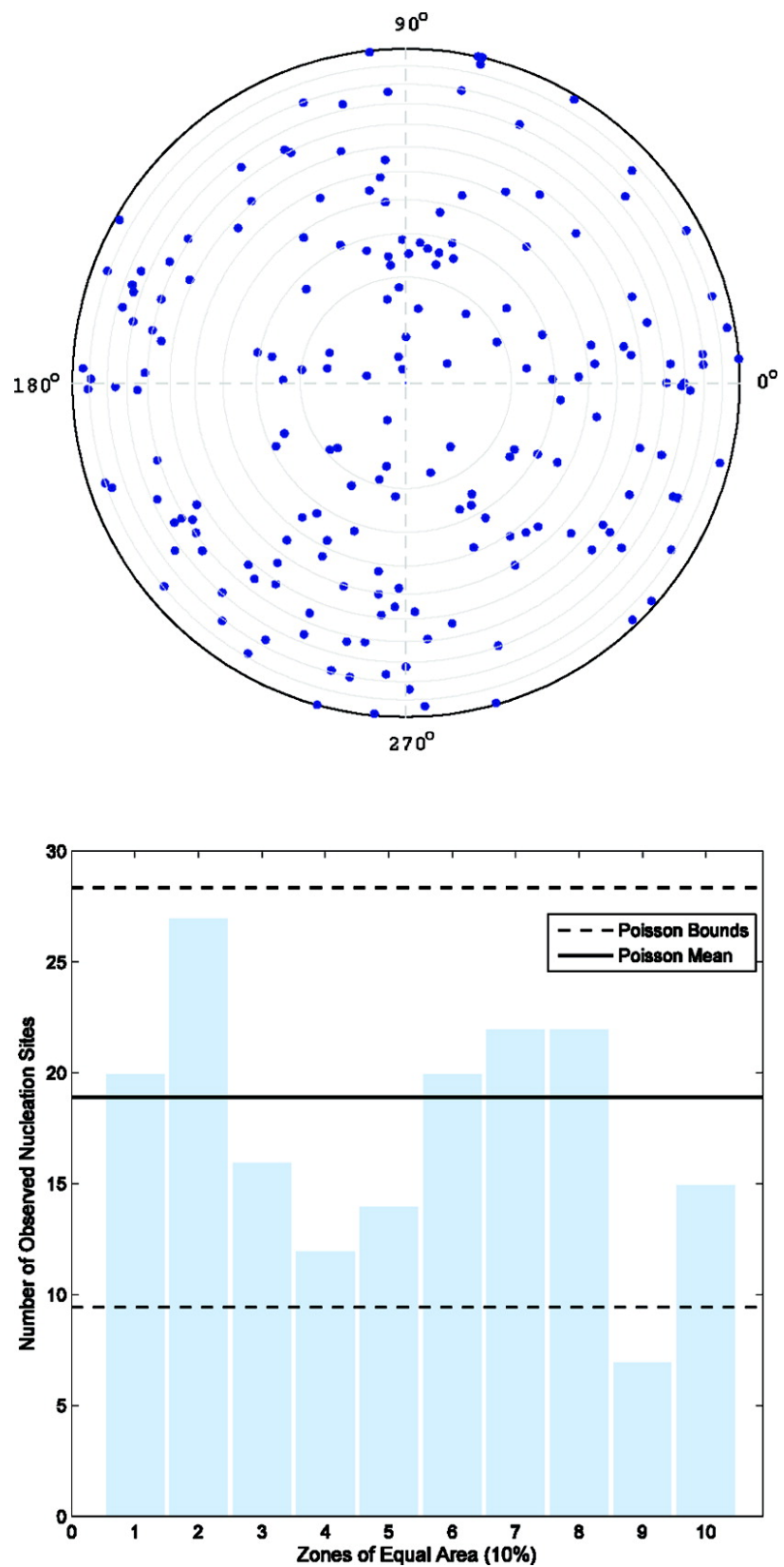

Figure 3.4: Evidence for spatial uniformity of nucleation events. Contrary to expectations, there is no preference for perimeter (contact mode) nucleation. (Top) Locations of the 189 freezing events on the horizontal plane. Radial positions have been scaled by drop radius to facilitate comparison between runs. (Bottom) An equal area histogram of 10 circular bands provides a quantitative measure of spatial uniformity. The solid horizontal line denotes the expected number of events per bin [4] assuming uniform distribution, and the dashed horizontal lines mark the Poisson bounds. 
On the basis of prior experiments, $[19,68,23,73]$ we anticipated nucleation to occur preferentially at the triple line. Thus, a surprising result of the current experiment is the observed statistical uniformity, the absence of preferential nucleation along the drop perimeter or "contact" mode. Could this result be attributed to an experimental flaw or an artifact (e.g., surface irregularities or dust on the substrate) acting as "preferred sites" for immersion nucleation? While this cannot be ruled out entirely, we observe that the nucleation site migrates around the substrate during repeated freezes of an individual droplet, which would not be expected if an energetically preferred nucleation site was present. Occasionally, we did observe consecutive, coincident-in-space freezing events, presumably due to external contamination. All such coincident events were eliminated from further analysis.

So why was surface crystallization observed in such a striking way in prior heterogeneous experiments[19, 68, 23, 73] but absent in the current study? Before confronting the notion of surface crystallization and the line tension conjecture prevalent in the recent literature, it is important to review the difference between this and earlier experiments. In some of the earlier experiments, the contact was highly localized,[19, 68, 23 but in the current study, the substrate is smooth, clean, and homogeneous. Perhaps, then, the localized contact somehow promotes nucleation in its vicinity. Support for this view can be found in a recent study where roughness of the substrate was the crucial characteristic[9]. Also, the heat sink and nucleating regions were decoupled in the current experiments, and thermal gradients were softened by using a very low cooling rate. Indeed, while Suzuki et al.[73] used a similar geometry (droplet on homogeneous silicon substrate), they used substrate cooling. Their main observation of preferential nucleation at the triple line is contrary to ours. Perhaps it is the thermal gradients and associated 
heat fluxes $[43,14]$ that somehow provoked or triggered nucleation in earlier experiments (although, for example, Shaw et al. and Fornea et al. observed similar enhancements for rather different cooling rates).

Another possibility of reconciling the current null result with prior observations from point-like contact experiments[19, 68, 23] is suggested by the vast difference in the extent of the contact line. If there exists a distinct nucleation rate for freezing at the triple line, it is presumably proportional to the contact length. The extensive nucleation rate (expressed as the number of freezing events per unit time) can then be written as the sum of contributions from immersion and contact modes $J_{i} \mathrm{~A}+J_{c} \mathrm{P}$, where $\mathrm{J}$ denotes intensive nucleation rate and $\mathrm{A}$ and $\mathrm{P}$ are the liquid-substrate area and air-liquid-substrate perimeter. The relative role of immersion versus triple line nucleation therefore scales with drop diameter in our geometry. Thus, decreasing the system size will tend to favor surface crystallization. Similarly, Duft and Leisner[18] pointed out the relevance of the surface to volume ratio to the question of surface crystallization in homogeneous nucleation. Their measurements of nucleation waiting time distributions allowed for an upper bound to be placed on the surface crystallization rate, but this is not possible in our work without appeal to a nucleation theory to provide the temperature dependence.

The preceding argument is purely geometric and need not involve details of the nucleation rates. However, one can specialize further. The energetic arguments of Djikaev and Ruckenstein[16] suggest a dependence of heterogeneous surface crystallization on the triple line tensions (ice-liquid-substrate, ice-air-substrate, liquid-air-substrate). An ice nucleus forming at the contact line presumably leads to a reduction in the nucleation barrier proportional to the product of the resulting change in line tension and the size of the 
nucleus. The possible importance of line tension for heterogeneous nucleation has also been pointed out before[21], but line tension values remain poorly characterized (even the sign[5] and existence[14]). We can gain some tentative insight through scale analysis; for a supercooled droplet (spherical cap geometry), the ratio of the line tension and surface tension contributions to free energy scales as $\tau / \sigma[5]$. Taking $\sigma \approx 0.1 \mathrm{~J} / \mathrm{m}^{2}$ and $\tau \approx$ $10^{-10} \mathrm{~J} / m,[62,50]$ the ratio $\tau / \sigma$ is on the order of $1 \mathrm{~nm}$. This hints that exceedingly small system sizes may be required before line tension becomes significant. In the prior experiments,[19, 68, 23] fine-scale imperfections on the ice-nucleating particles likely existed, and line tension may have been important. The question remains open.

\subsection{Experimental Methods}

The experiment design (shown schematically in Figure 3.1, left panel) consists of a cylindrical volume with copper-lined, actively cooled walls and a small optical window at the top. Wall temperatures are regulated via a circulated ethelyne glycol solution from a Neslab RTE-140 Chiller. A humidified nitrogen supply line can increase the relative humidity when $\mathrm{T}>273 \mathrm{~K}$, but sample runs are typically limited by evaporation to 12-20 freeze-thaw cycles before the droplet must be replaced. The temperature and humidity within the volume are recorded with a RTD (via a Lakeshore 331), and a capacitive polymer sensor (Precon HS-2000D). Our experiments were performed with both p- and n-doped 2.5 cm diameter atomically smooth silicon wafers (Wafer World, Inc., test grade, 250-300 $\mu \mathrm{m}$ thick). No difference in nucleating properties between $\mathrm{p}$ and $\mathrm{n}$ substrates was observed. The silicon wafers were washed with copious amounts of distilled water and then acetone and dried with a clean nitrogen flow. After the wafer was placed in the chamber, a 5 or $10 \mu \mathrm{L}$ droplet of type 1 grade water (distilled, deionized, UV-irradiated) was placed on its 
center, and the chamber was sealed. Lastly, a humidified nitrogen flow of $1 \mathrm{~L} / \mathrm{min}$ was used to purge the chamber, prior to initiation of the chilling cycle.

In practice, the chamber is cooled in two stages, (i) quick cooling to minimize evaporation $(\lambda=0.3-0.5 \mathrm{~K} / \mathrm{min})$ and, once the likely freezing range is attained, (ii) a slower cooling rate $(\lambda=0.13-0.25 \mathrm{~K} / \mathrm{min})$, without airflow into the volume. The notion of thermal relaxation time can be applied to the chamber as a whole. The thermal diffusivity of air is approximately $0.2 \mathrm{~cm}^{2} / \mathrm{sec}$, so that for our chamber dimensions of $4 \mathrm{~cm}$, the temperature difference between the wall and the interior is about $1 \mathrm{~K}$. This lag of $\lambda \tau_{\text {air }} \approx 1 \mathrm{~K}$ between the actively cooled walls and the interior is indeed confirmed by simultaneous temperature measurements.

We recorded a video of each freeze and determined the location of the initial nucleation site. A high-speed Phantom v7.3 camera continuously recorded the droplet (through the window above the droplet; see Figure 3.1) when the chamberâĂŹs temperature entered the freezing regime. Illumination was provided by LEDs suspended from the top section of the chamber. We have also developed an optical triggering system, which initiates a transfer of the images in the camera's ring buffer when a freezing event is detected. After the data are transferred, a heating cycle warms the chamber volume to $276 \mathrm{~K}$ to melt the droplet before the cooling cycle is repeated.

The observed mean freezing temperature of the drops is $-18^{\circ} \mathrm{C}$. The chamber contains a water reservoir that freezes earlier than the drop (at approximately $-5^{\circ} \mathrm{C}$ ); therefore, at the time of drop freezing, the relative humidity is close to $100 \%$ with respect to ice. The drops slowly evaporate during the repeated freezing and melting cycles, losing roughly 
half of their mass over a $36 \mathrm{~h}$ period. An upper bound on the resulting evaporative cooling rate, obtained by neglecting heat transfer into the drop from the air or substrate,

can be estimated as $(\mathrm{L} / \mathrm{c})(1 / \mathrm{m})\left(\frac{\partial m}{\partial t}\right) \approx 0.1 \mathrm{~K} / \mathrm{min}$, where $\mathrm{L}$ and $\mathrm{c}$ are the latent heat of vaporization and the specific heat of water, respectively, and $\mathrm{m}$ is the drop mass. This is of the same order as the chamber cooling rate, and in practice, the thermal gradients driving heat transfer are overwhelmingly confined to the surrounding air rather than the water because the thermal diffusivity of air is greater by a factor of approximately 100 . Heating due to the LED illumination is estimated from the emission spectrum and the wavelength-dependent complex refractive index of water[6]. Unlike evaporation, the heating is uniform throughout the drop because of the very low absorption coefficient of water. Accounting for the LED efficiency, the resulting heating rate for a $10 \mu \mathrm{L}$ droplet is approximately $0.02 \mathrm{~K} / \mathrm{min}$, roughly an order of magnitude less than the chamber cooling rate.

\subsection{Acknowledgement}

The authors would like to thank E. Bodenschatz and Z. Warhaft (Cornell University) for use of the Phantom v7 camera. Helpful discussions with W. Cantrell and R. Sear are appreciated. A.B.K. wishes to thank the staff of Environmental Sciences Department, Weizmann Institute of Science, for hospitality and support. This research was supported in part by an award from the Department of Energy (DOE) Office of Science Graduate Fellowship Program administered by the Oak Ridge Institute for Science and Education for the DOE. ORISE is managed by Oak Ridge Associated Universities (ORAU) under DOE Contract Number DE-AC05-06OR23100. All opinions expressed in this paper are the authors' and do not necessarily reflect the policies and views of DOE, ORAU, or 
ORISE. This work was also supported in part by the National Science Foundation, Grants ATM05-54670 and AGS-1026123. 


\section{Chapter 4}

\section{The Role of Thermal Gradients}

\section{High Speed Imaging of Freezing Drops: Still no Preference for the Contact Line [32]}

This chapter details a second study of the role of rapid cooling rates and varying contact angles on heterogeneous nucleation, and was based on a research collaboration ${ }^{1}$. This work is published in full form in the Journal of Physical Chemistry $\mathrm{C}^{2}$, and is reprinted with permission by the American Chemical Society.

\footnotetext{
${ }^{1}$ This paper is co-authored by A. B. Kostinski \& R. A. Shaw.

${ }^{2}$ Reprinted with permission from:

Gurganus, Colin, Kostinski, Alexander B and Shaw, Raymond A, The Journal of Physical Chemistry C, 117, 6195-6200, 2013 . Copyright 2013 American Chemical Society
} 


\subsection{Abstract}

Recent experiments by Suzuki et al. [73] and Gurganus et al. [31] on liquid-solid nucleation arrived at conflicting results regarding a preferred status of the triple line between water, air, and an ice-catalyzing substrate. Temperature nonuniformity within drops and substrate dependent contact angles have been suggested as culprits in a recent review by Sear [66]. To that end, we redesigned our earlier experiment to allow substrate-induced cooling and a side view with a second high-speed camera. The two camera views pinpoint the spatial location of nucleation sites in both the vertical and horizontal directions. Here we report such nucleation positioning results measured within drops freezing on a substrate. The role of thermal gradients was explored in three ways: (i) implementing direct cooling of the substrate; (ii) mimicking (higher) cooling rates used by Suzuki et al.; and (iii) varying the drop-substrate contact angle. No influence of thermal gradients on the preference for freezing at the triple line has been observed. Thermal simulations of the drop-substrate system conïn̆Arm that horizontal temperature gradients are extremely small. Furthermore, treatment of the substrate to obtain a range of contact angles also yielded no preference for freezing at the triple line. The combined top and side views of the freezing drops suggest that apparent triple-line nucleation can be a spurious result of the viewing geometry. 


\subsection{Introduction}

Heterogeneous nucleation in clouds converts water to ice, thereby affecting weather and climate. Probably, the least understood heterogeneous nucleation pathway is the contact mode, where a catalyst particle causes freezing by contact with a supercooled droplet.[66] Recent laboratory experiments with 10-100 $\mu \mathrm{m}$ particles have indicated that contact promotes nucleation even when the catalyst resides within the liquid drop but in the immediate vicinity of the air-water interface. $[19,68,23]$ In an attempt to interpret these experimental results, thermodynamic arguments [16] and computational studies [64] have suggested that nucleation may be preferred near the three-phase interface or triple line.

To examine the role of the triple line enhancement, Gurganus et al. [31] distilled the problem into a simple, drop-on-substrate geometry so that the spatial distribution of nucleation sites could be pinpointed. Contrary to expectations, in our first study no preference for the triple line was observed. This is in contrast to a similar experiment by Suzuki et al., [73] who did observe preferential nucleation at the triple line. The apparent discrepancy between the two experiments has been highlighted in a recent review by Sear[66], where attention was drawn to differences in drop-substrate contact angle and possible thermal biases induced by different cooling methods. Specifically, Sear suggested that "A set of experiments in which a parameter (cooling rate?...) is varied and the system

goes from nucleation at the contact line to nucleation away from this line, would be very helpful. " [66] To that end, we have carried out a set of follow-up experiments where we changed the method and rate of cooling and varied drop-substrate contact angles. 


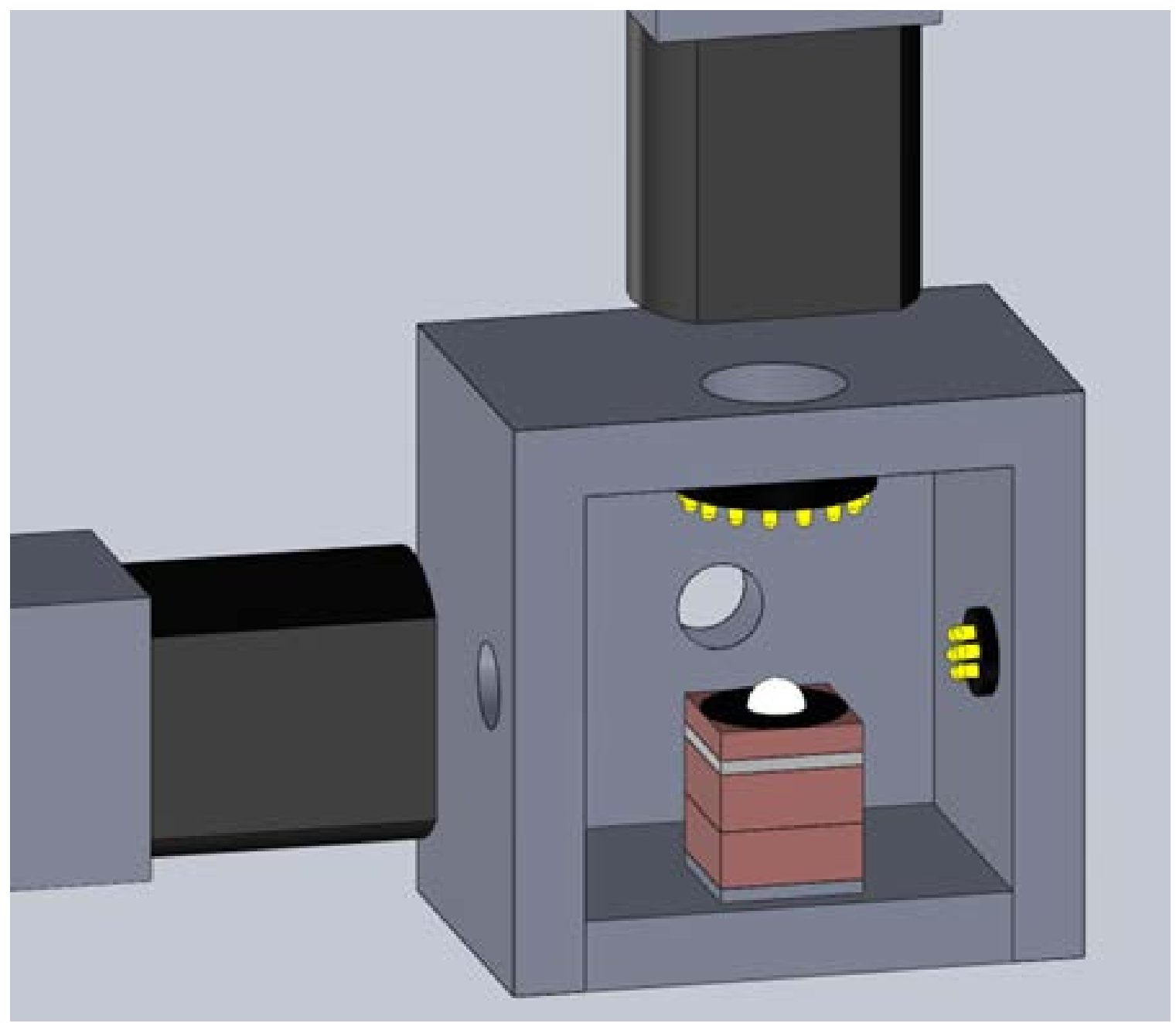

Figure 4.1: Schematic of the new thermal chamber. Continuing the geometry of refs 1 and 2, this study examines supercooled droplets in contact with an atomically smooth Si substrate. Unlike our prior study, the substrate is placed in thermal contact with a Peltier cooling element (white) to achieve high cooling rates $(5 \mathrm{~K} / \mathrm{min})$. The droplet and cooling element are contained in an isothermal chamber with positive pressure from a dry air line. Optical ports on the top and sides of the chamber allow for simultaneous imaging of the droplet in the horizontal and vertical planes.

The two experiments[73, 31] differ in the method of cooling employed, in the water volume studied, and in the form of monitoring the drop freezing. In our prior study we took extreme care to minimize thermal gradients or biases by indirectly cooling the nearly isothermal volume at a very gradual rate of $<0.5 \mathrm{~K} / \mathrm{min}$. As is commonly done in other 
studies,[19, 68, 23, 53] Suzuki et al.[73] employed a substrate cooling technique with a rate of approximately $5 \mathrm{~K} / \mathrm{min}$. Such rates can occur in the atmosphere within strong updrafts. It has been suggested that prior studies may have shown a bias in favor of surface crystallization because such high cooling rates will lead to development of a steady state thermal gradient across the droplet.[31] If this gradient exists across the droplet-substrate interface then we might expect to see preferential nucleation at the periphery where the thermal load is reduced (in spherical cap geometry). Finally, in our prior experiment the droplet crystallization was monitored with a camera located above the water drop, whereas in the Suzuki et al. study a side-viewing camera was used. In this work we have introduced substrate cooling at comparably high rates as are typical in other experiments, and we have added a second, side-viewing camera to further pinpoint the nucleation location and to continuously measure the drop-substrate contact angle.

\subsection{Experimental Techniques}

The basic experimental approach is similar to our prior study; namely, the location of an ice nucleation event is identified by observing the initial freezing of a water drop with a high-speed camera: when the freezing movie is played in reverse, eventually the point of origin of the ice crystal can be determined, including whether the point lies at the substrate-water interface or along the substrate-water-air contact line (the triple line). To simplify the interfacial geometry, we utilize homogeneous and atomically smooth 50 $\mathrm{mm}$ test grade silicon substrates for our nucleation medium. Using an atomic force microscope, we record root mean squared (RMS) surface roughness values of $<10 \mathrm{~nm}$ for both new and cleaned (acetone and isopropyl alcohol (IPA) then dried with a nitrogen spray) wafers. Similar to our prior study,[31] in these experiments $30 \mu \mathrm{L}$ water droplets 
(distilled, deionized, UV-irradiated) are placed on the substrate with a syringe.

For this study, a larger test chamber was built, with optical ports on the top and side to allow for simultaneous imaging of the top and side of the droplet with a synchronized pair of Photron SA2 cameras. Sufficient lighting for $5-10 \mathrm{kHz}$ sampling is provided by arrays of blue-white LED arrays above and behind the droplet, which contribute negligibly to droplet heating.[31] The overall geometry is shown schematically in Figure 4.1. The cameras continuously record the droplet during the cooling cycle through a $10 \mathrm{sec}$ ring buffer. An IR thermocouple monitors the droplet surface temperature and is used to trigger a transfer of the camera buffer when a latent heat spike is observed. This technique[31] allows us to record the entire freezing event and to isolate the position of each nucleation site with Image J image analysis software, in both the top and side views as shown in Figure 4.2 .

In an attempt to maximize the possible role of thermal gradients in the droplet we selected a large drop size $(30 \mu \mathrm{L})$ and a cooling rate $(5 \mathrm{~K} / \mathrm{min})$, both similar to the experimental conditions of Suzuki et al.[73] The increased cooling rate was achieved by the addition of a solid state cooling element (Peltier element) in the center of the test chamber

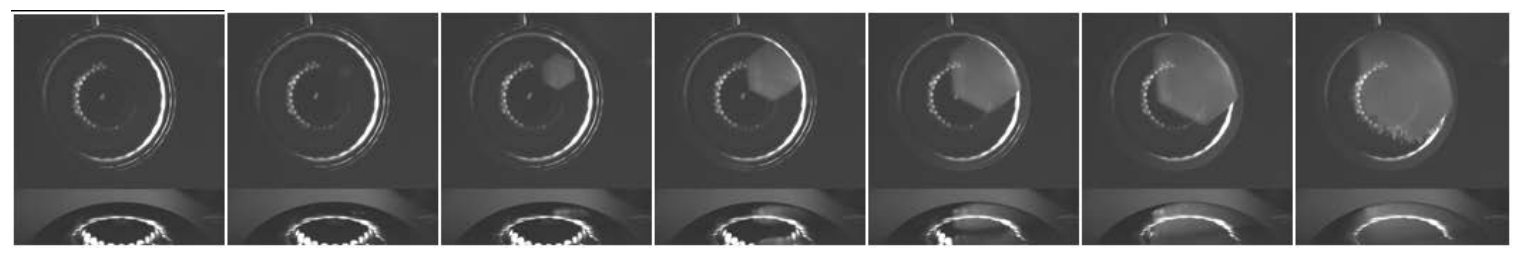

Figure 4.2: Pair of high-speed Photron SA2 cameras allows us to pinpoint nucleation sites and measure the droplet contact angle. In this film strip each frame represents a time step of $2 \mathrm{msec}$, or every 20th frame at a sample rate of $10 \mathrm{kHz}$. The nucleation site is evident at the center of the hexagonal crystal when the film is viewed in reverse. 
(Figure 4.1). The chamber is maintained at a steady temperature (to within $\pm 0.1 \mathrm{~K}$ ) by circulation of a chilled glycol solution with a Neslab RE 140 chiller. The temperature of the substrate is set with the Peltier chiller through a PID interface with an RTD probe underneath the substrate.

In a typical experiment, each droplet in the chamber is frozen and thawed 10 to 20 times as part of a 30 min automated cooling and heating cycle. After the droplet melts, the camera buffers are triggered to begin recording again, and the cooling cycle repeats. To prevent icing of the chamber walls, a positive pressure is maintained in the chamber volume with a dry air supply at $2-5 \mathrm{~L} / \mathrm{min}$. Because the chamber air is subsaturated, the droplet will continuously evaporate on the substrate, typically losing half of its mass over the duration of the experiment. To better compare the location of nucleation sites between different drops, the droplet diameter is measured after each freeze to normalize the radial position of the nucleation site. During this evaporation process, the drop-substrate contact angle slowly decreases because of drop pinning, and this contact angle is measured with the side-view camera. Furthermore, the contact angle was changed over a larger range by using clean silicon wafers in one set of experiments and silanized silicon wafers in another set of experiments. The resulting variation allows the possible dependence of freezing position on contact angle to be investigated.

\subsection{Results}

The top-view movie of each freezing event was used to determine the nucleation site on the substrate, and the side-view movie was used to determine the contact angle of the 
droplet. Because the droplet acts as a liquid lens, the exact determination of the freezing site is complicated, especially in the case of the side view (see Supporting Information). In some data runs a small dust particle or $\mathrm{Si}$ wafer defect that cannot be resolved will induce nucleation preferentially at one location. We do not believe that these defects are significant in many experiments, as typical surface roughness values of clean and used wafers have both been shown to have surface roughness values of $10 \mathrm{~nm}$. Such consecutive nucleation events at the same site are removed from further processing. We also note that the side view shows no evidence of nucleation occurring above the substrate, and this is further confirmed by consistently horizontal (parallel to substrate) orientation of growing ice crystals.

The spatial distribution of freezing sites for droplets on the clean Si wafer cooled through the substrate at a rate of approximately $5 \mathrm{~K} / \mathrm{min}$ is shown in the left panel of Figure 4.3. As in the prior results[31], no preference for the triple line is observed. This observation was somewhat surprising, given our initial expectations about the role of thermal gradients. The same holds true when the water drop is placed on a silanized Si wafer, resulting in a steeper contact angle, as shown in the right panel of Figure 4.3. Despite increasing the cooling rate by an order of magnitude, we see no apparent change in the distribution of freezing sites from our prior study.[31] This would suggest that any thermal bias in the experiment does not play a significant role in nucleation.

The role of contact angle can be further studied. The twin imaging technique allows us to correlate the radial position of a nucleation germ site with corresponding contact angle of the droplet on the test substrate (Figure 4.4). The large spread in droplet contact angles $\left(30^{\circ} \rightarrow 90^{\circ}\right)$ appears to have no correlation with the radial distribution of freezing sites. 

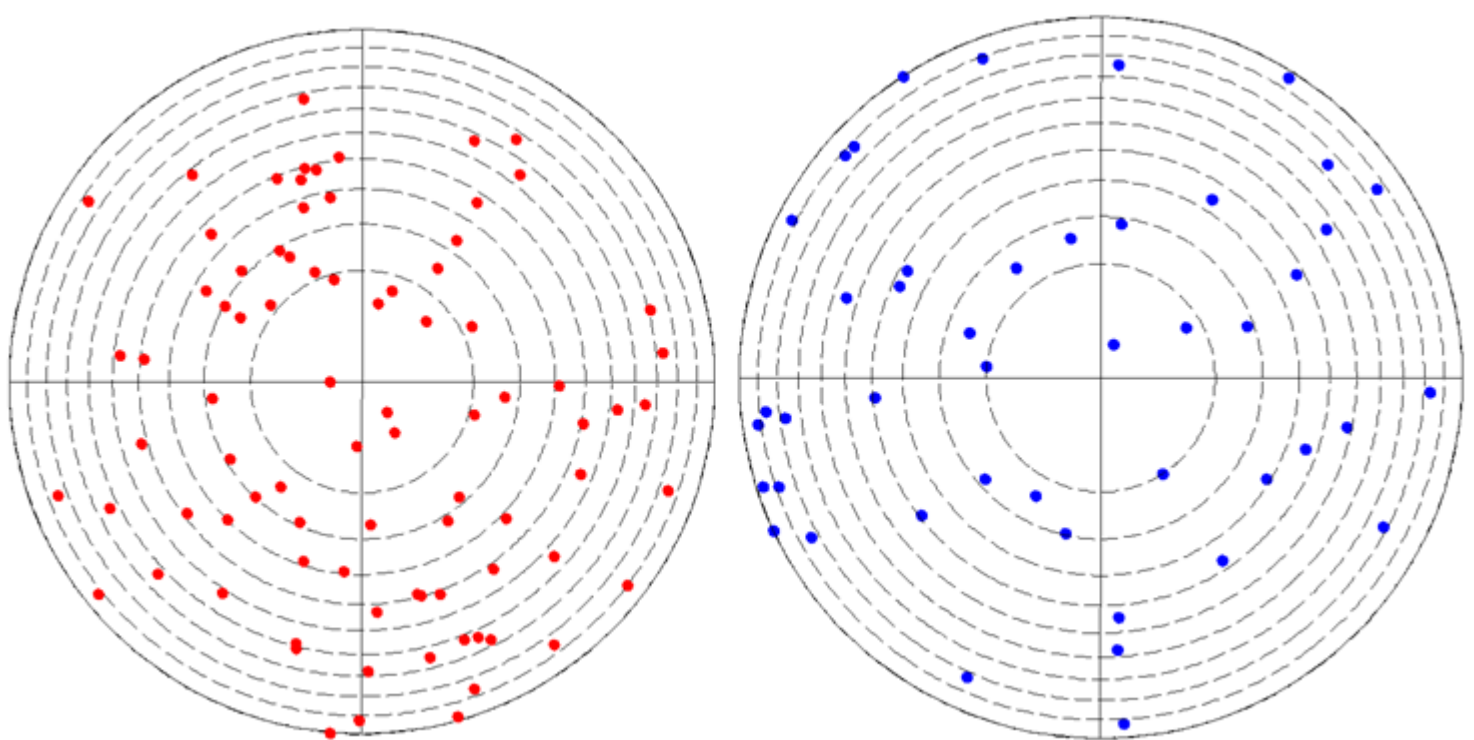

Figure 4.3: From image analysis of the top-view movies, we can determine the nucleation site at the droplet-substrate interface. In the left panel, nucleation sites for a droplet with low contact angles on a clean silicon wafer are displayed in red. In the right panel, nucleation sites for a droplet with higher contact angles on a silanized wafer are displayed in blue. The dashed lines separate regions of equal area (10\%).[31] Both distributions appear uniformly random in agreement with our previous observations

\subsection{Discussion}

Beginning with Gurganus et al. we developed an experimental approach that disentangles the notion of line tension from the contact and has a simple, symmetric, and clearly defined triple line.[31] Unlike most studies, the experiments address nucleation directly in terms of the spatial distribution of nucleation sites rather than in terms of temperature dependence. Here we have tested the conjectures that differences in drop-substrate contact angle or thermal biases may cause a preference for freezing at the triple line.[31, 66] In an attempt to better understand the role of thermal gradients in our system, we have modeled the droplet substrate geometry using the Solidworks thermal 


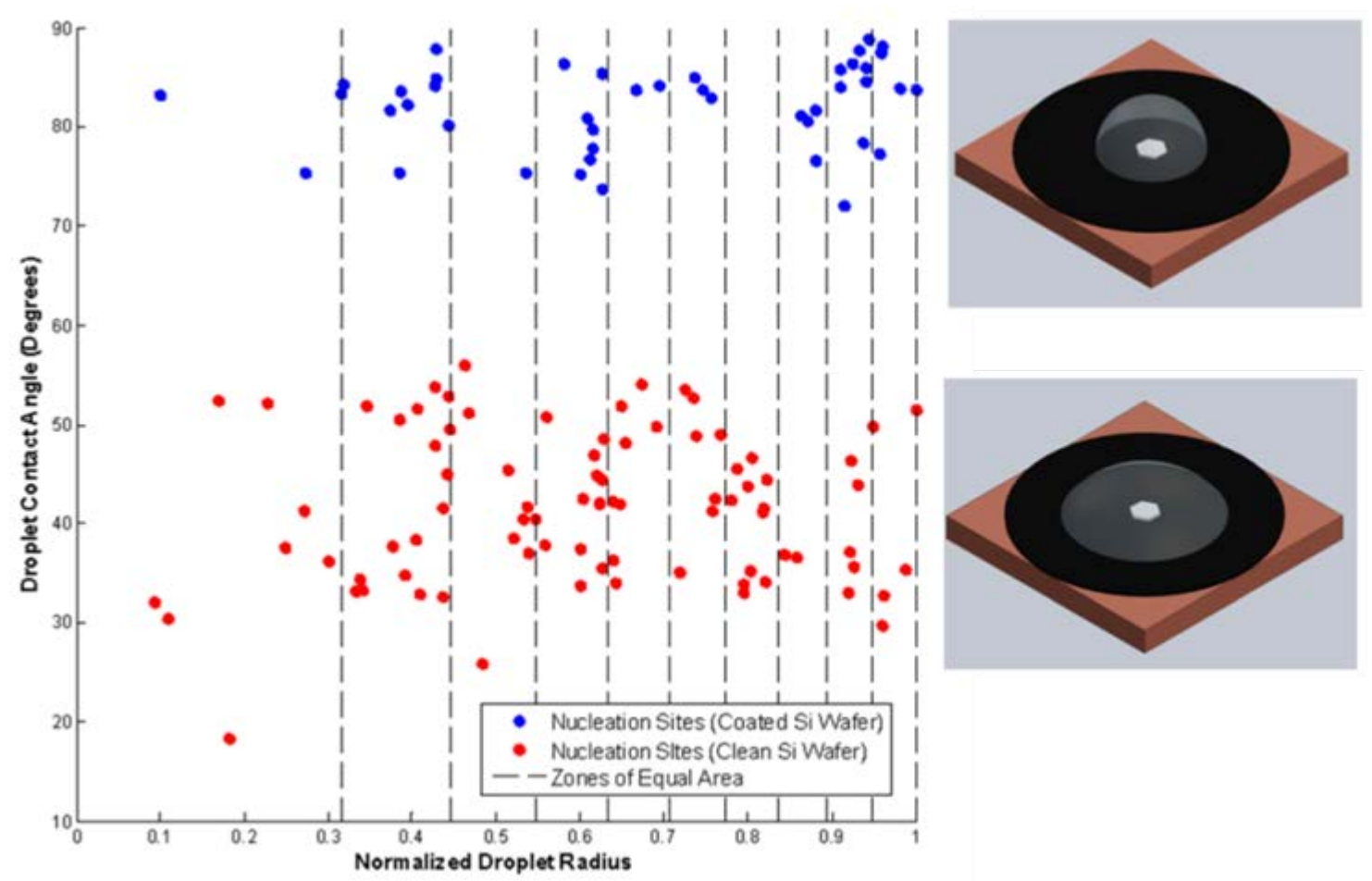

Figure 4.4: Image analysis from the side-view camera allows us to determine the contact angle of each droplet at the initiation of freezing. The red points indicate observed nucleation events on a clean Si wafer, while the blue points indicate observed nucleation sites on a silanized Si wafer. As in Figure 4.3, the dashed lines separate 10 zones on equal area to help guide the eye in the radial distribution of sites. The large variability in the observed contact angles of both sets is caused by pinning at the triple line during slow evaporation of the droplet. The approximate ranges for the observed drop contact angle, perimeter, and height are respectively $30^{\circ}-90^{\circ}, 10-30 \mathrm{~mm}$, and $1-2.5 \mathrm{~mm}$. There appears to be no difference in the distribution of nucleation sites with contact angle.

simulation software package. In this model a $2.5 \mathrm{~mm}$ hemispherical water droplet (30 $\mu \mathrm{L})$ resting on a $500 \mu \mathrm{m}$ thick silicon wafer is placed in thermal contact with a $10 \mathrm{~mm}$ copper block (Figure 4.5). An automated meshing algorithm mapped the system and established a nodal spacing of $100 \mu \mathrm{m}$. To begin, all elements in the system are held at an equilibrium temperature of $275 \mathrm{~K}$. To simulate the effect of the solid state cooling element the temperature of the bottom plane of the copper block is decreased uniformly at a rate of $5 \mathrm{~K} / \mathrm{min}$, for $360 \mathrm{sec}$. These conditions are similar to those within our chamber. 


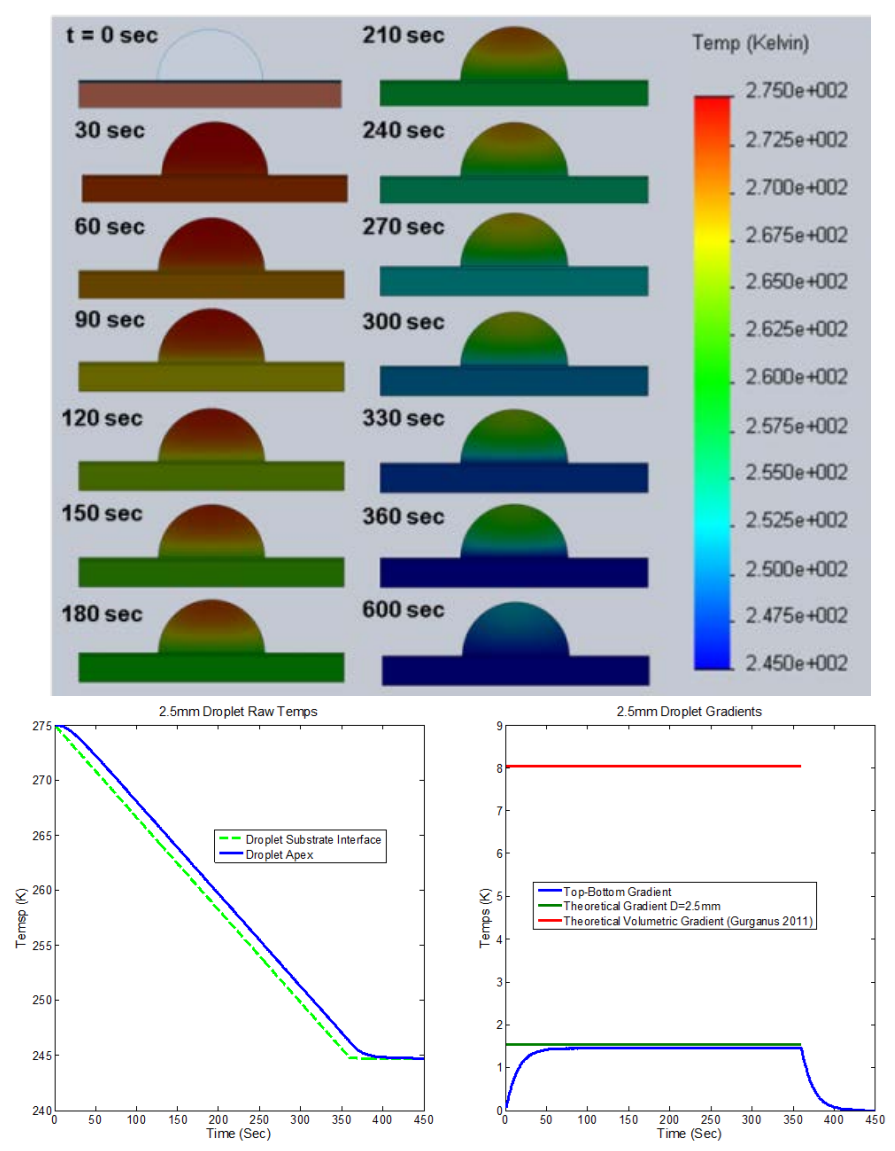

Figure 4.5: Results of our thermal analysis of this geometry using the Solidworks software package. This simulation mimics our experimental conditions, with the droplet, wafer, and heat sink initially at a stable equilibrium with the isothermal chamber $(275 \mathrm{~K})$. At $\mathrm{t}=0$, the temperature of the heat sink is decreased at a rate of $5 \mathrm{~K} / \mathrm{min}$ for $360 \mathrm{~s}$ until it falls to a substrate temperature of $245 \mathrm{~K}$, at which the temperature of the heat sink is held constant until the droplet comes into equilibrium. The bottom panels represent data from nodes at the top and bottom of the droplet. The first node is on the droplet-silicon interface. The selection of this bottom node is arbitrary because we observe no thermal gradient at the droplet-substrate interface. The second node is located at the apex of the droplet $2.5 \mathrm{~mm}$ above the interface. The left panel displays the raw temperature at both nodes during the cooling cycle. The right panel displays the thermal gradient that develops between these two nodes. After an initial transient stage $(60 \mathrm{~s})$, a steady state gradient of $1.5 \mathrm{~K}$ is maintained throughout the cooling cycle. After the active cooling ends (360 s), the droplet relaxes exponentially toward the new equilibrium temperature. 
Several simplifying assumptions are made in the model. First, radiation is neglected due to the relative speed of the cooling process. Second, internal circulation and convection within the drop is neglected. There is no external forcing or oscillation (the droplet is pinned to an interface, and minimal air exchange is used), and the simulated thermal gradients are small: with a water droplet diameter of $2.5 \mathrm{~mm}$ and a temperature difference of $\Delta \mathrm{T}=1.5 \mathrm{~K}$, we obtain a Rayleigh number of $\mathrm{Ra}=\frac{g \beta \Delta T H^{3}}{v \alpha}=102$, well below the critical value for the onset of convection (where $\mathrm{g}$ is the gravitational acceleration, $\beta$ is the thermal expansion coefficient of water, $v$ is the kinematic viscosity of water, $\alpha$ is the thermal diffusivity of water, and $\mathrm{H}$ is the drop height). Hence, we can safely model the drop via the heat equation.

The simulation results are shown in Figure 4.5. The higher thermal diffusivity of copper and silicon allows the substrate to relax quickly to the cooling element, and we see virtually no gradient across this region. Within the droplet volume, we observe a temperature gradient develop in the vertical direction, due to the lower thermal diffusivity of water. The temperature difference scales with the depth of the liquid layer, attaining 1.5 $\mathrm{K}$ between the apex of the droplet and substrate after one droplet relaxation time $\tau$ (Figure 4.5, bottom left). In an earlier publication, we proposed a characteristic thermal variation parameter $\Delta \mathrm{T} \approx \lambda \tau$, where $\lambda$ is the system cooling rate.[31] To compare different experimental geometries, the time scale for diffusion was characterized by the liquid volume for a spherical droplet $\tau \approx \frac{V^{2 / 3}}{\alpha}$, where $\alpha$ is the thermal diffusivity of water. Using this formulation, the temperature difference across the droplet has the correct order of magnitude but is overestimated $(\Delta \mathrm{T} \approx 8 \mathrm{~K})$ because of the spherical cap geometry (Figure 4.5, bottom right). Instead, by substituting the depth of the fluid (h) into the equation for thermal relaxation $\tau \approx \frac{h^{2}}{\alpha}$, the predicted steady state gradient agrees well with the 
simulation. The important observation for interpretation of the nucleation measurements, however, is that the temperature over the base of the droplet is uniform to within our temperature resolution $\left(10^{-2} \mathrm{~K}\right)$. As noted in our previous work,[31] this value is within the $0.1 \mathrm{~K}$ tolerance for uniform nucleation based on the classical nucleation theory.[55] Therefore, a negligible bias in nucleation rate occurs even for relatively strong cooling rates. Furthermore, neither surface tension nor contact angle dependence on temperature is likely to play a significant role under these circumstances.[14]

If cooling rate and contact angle do not shed any light on the contrasting results of Suzuki et al.[73] and Gurganus et al.[31], is there another possible explanation? The addition of the side-view camera may provide a hint. As can be seen in the accompanying video (Supporting Information), even when the point of nucleation is clearly observed to be far from the triple line in the overhead view of the droplet, the side view gives the impression that the freezing initiates at the triple line. The same difference in perceptions can be seen to some extent in Figure 4.2. This effect is not to be confused with the reflected image of the ice formation in the top of the drop, which also can be seen in both Figure 4.2 and the movie. There is no proof that this optical artifact explains the difference in observations, but the results of this study stand on their own, independently: over the ranges studied, variations in drop cooling rate and in drop-substrate geometry do not change the prior reported results.[31] There is still no preference for the triple line.

So what are the implications of these results to the observed "contact" nucleation phenomenon[7,53] and its possible relevance to the atmosphere?[57,59] Indeed a skeptical reader might object that the simple geometry explored here has little to do with an aerosol particle nucleating a supercooled droplet by contact. For example, transiency of 
contact, microscopic roughness,[65, 40] or molecular interactions[78] may play a role. However, this simple geometry was developed precisely to single out and test the following conjecture: Does a triple line formed at the aerosol-droplet-air intersection promote nucleation? To that end, this experiment would suggest no, at least not at macroscopic length scales.

\subsection{Acknowledgement}

Helpful discussions with W. Cantrell and R. Sear are appreciated. This research was supported in part by an award from the Department of Energy (DOE) Office of Science Graduate Fellowship Program administered by the Oak Ridge Institute for Science and Education for the DOE. ORISE is managed by Oak Ridge Associated Universities (ORAU) under DOE Contract Number DE-AC05-06OR23100. All opinions expressed in this paper are the authors' and do not necessarily reflect the policies and views of DOE, ORAU, or ORISE. This work was also supported in part by the National Science Foundation AGS-1119164 and by the DOE Atmospheric System Research program. The authors would like to thank J. Drelich for his consultation on AFM measurements. 


\section{Chapter 5}

\section{The Role of Surface Texture}

\section{Ice nucleation transition to the contact line observed on nano-textured surfaces [30]}

This chapter details a experiments preformed to examine the role that surface texture plays in surface nucleation, and was based on a research collaboration ${ }^{1}$. This work will be published in full form ${ }^{2}$, with minor revisions in Physical Review Letters and is reprinted with permission by the American Physical Society.

\footnotetext{
${ }^{1}$ This paper is co-authored by J. C. Charnawskas, A. B. Kostinski \& R. A. Shaw.

${ }^{2}$ Reprinted with permission from:

C. Gurganus, J. C. Charnawskas, A. B. Kostinski \& R. A. Shaw., Physical Review Letters, 113, 235701, 2014. "Copyright 2014 by the American Physical Society."
} 


\subsection{Abstract}

It has been conjectured that roughness plays a role in surface nucleation, the tendency for freezing to begin preferentially at the liquid-gas interface. Using high speed imaging, we sought evidence for freezing at the contact line on catalyst substrates with imposed characteristic length scales (texture). Length scales consistent with the critical nucleus size and with $\delta \sim \tau / \sigma$, where $\tau$ is a relevant line tension and $\sigma$ is the surface tension, range from nanometers to micron. It is found that nano-scale texture causes a shift in the nucleation of ice in supercooled water to the three-phase contact line, while micro-scale texture does not.

\subsection{Introduction}

While nucleation of solids in supercooled liquids is ubiquitous[15, 65, 66], surface nucleation, the tendency for freezing to begin preferentially at the liquid-gas interface, has remained puzzling[74, 18, 68, 69, 51, 64, 72, 16,9]. Furthermore, in the presence of foreign catalysts the associated heterogeneous nucleation has been observed to prefer the three-phase contact line (triple line), especially for small particles [19] and rough surfaces [9]. Motivated by the conjectured importance of roughness and the contact line, we have searched for evidence of a shift to surface nucleation as the characteristic roughness length scale is decreased. Two plausible length scales associated with heterogeneous nucleation, the critical radius for a nucleation seed, and the length scale at which linear and surface energies are comparable, yield a range from micrometers to nanometers. In this Letter we 
show, using high speed imaging of the transient freezing process in supercooled water, that nano-scale texture causes a shift in the nucleation to the three-phase contact line, while micro-scale texture does not. Both the mean and variance of the freezing temperature are observed to increase, also pointing to the importance of nanotexture given that variances of independent causes add. The possibility of a transition or optimal length scale has implications for the effectiveness of nucleation catalysts, including formation of ice in atmospheric clouds[7].

Uniform probability of freezing is a standard assumption in nucleation theory: probability scaling as the volume of supercooled liquid for homogeneous nucleation, or as the area of the liquid-catalyst interface for heterogeneous nucleation. Recent studies suggest that for systems as widely varying as atomic liquids[51], salts[2], tetrahedral liquids[45], hexaflourides[11], metal alloys[69, 72], Nickel-Silicon[48], polymers[8], and water[74, 17], homogeneous nucleation prefers the liquid-vapor interface, and therefore its rate scales not as volume but rather as area. The mechanism for surface nucleation remains unclear, and even more troubling, its predominance has been qualified and questioned[18, 70, 42, 76]. Meanwhile, experiments on the nucleation of ice on small particles in supercooled water have revealed a strong enhancement in nucleation rate for particles at the liquid-water - air interface[68, 23], suggesting that whatever physics underlies surface nucleation likely extends to heterogeneous nucleation as well. Sorting out this mystery is more than academic because it addresses fundamental aspects of classical nucleation theory (CNT) and thus predictability of nucleation processes; some long standing puzzles such as the empirical observation that 'contact nucleation' is more efficient than 'immersion nucleation' in supercooled cloud droplets ${ }^{3}$ may well be

${ }^{3}$ Contact and immersion ice nucleation are typically defined as nucleation of ice with a catalyst particle at the air-liquid interface or immersed in liquid water, respectively [7]. 
intertwined with the physics of surface nucleation. A leading hypothesis for the preference for surface nucleation is the formation of a three-phase interface[64, 16], and this aspect is investigated here for heterogeneous nucleation of ice in supercooled water.

Rough [9] or 'point-like contact' [19] nucleation catalysts have been observed to induce nucleation at the three-phase contact (triple) line. It has been suggested that a free energy per unit length or line tension $\tau$ for the contact line contributes to the nucleation kinetics [16]. Thus, an extensive nucleation rate (number of freezing events per unit time) would be a sum of contributions from immersion and contact modes. In recent work we sought direct confirmation by observing the freezing of mm-sized supercooled water droplets on atomically smooth substrates using high speed optical imaging: and yet for a variety of contact angles and cooling rates, no preference for nucleation at the macroscopic air-water-substrate contact line was observed [31, 32]. It is possible, however, that the lack of contact-line-nucleation in those experiments reflects the system geometry. For example, an extensive nucleation rate dependent on both droplet-substrate surface area and perimeter would lead to the relative role of immersion versus contact line nucleation scaling with drop diameter. If so, then decreasing the drop size should favor surface nucleation. Rather than decreasing the size of the supercooled liquid volume, which renders our high-speed imaging method more difficult, here we modify the geometry of the nucleation catalyst so as to impose 'textures' exhibiting a range of length scales on the air-water-substrate contact line. The question is whether catalyst geometry alone can induce a preference for nucleation at the contact line.

The apparent role of substrate geometry and roughness $[9,15]$ motivates a consideration of possible length scales that could enter the heterogeneous nucleation problem. One is 
the size of the critical nucleus predicted by CNT. It has been shown $[10,58,77]$ that steps, pores, cracks, or other surface features with sizes on the order of the critical nucleus may promote more efficient nucleation by lowering the free energy barrier. For example, Page et al.[58] demonstrated that a two-step nucleation rate exists for ice within and outside of a pore, and therefore an optimal pore size exists, near the critical nucleus size, at which nucleation rate is maximized. Quite generally, the critical radius for nucleation is obtained from the Gibbs-Thomson equation $r^{\star}=2 \sigma v_{i} / \Delta \mu$, where $v_{i}$ is the molecular volume for ice and $\Delta \mu$ is the chemical potential difference between the supercooled liquid and the nucleated solid. It can be expressed as $\Delta \mu=k T \ln p_{w} / p_{i} \approx l_{f} \Delta T / T_{0}$, where $p_{w}$ and $p_{i}$ are the equilibrium vapor pressures of liquid water and ice, respectively, $l_{f}$ is the latent heat of fusion, $T_{0}$ is the melting temperature, and $\Delta T \equiv T_{0}-T$ is the supercooling temperature. For the typical $\Delta T$ of 5 to $35 \mathrm{~K}$, the critical radius varies over the approximate range $r^{\star} \approx 1$ to $10 \mathrm{~nm}$. In the experiments reported here, the observed supercooling temperatures suggest a length scale $\lambda \approx 2 r^{\star} \approx 10 \mathrm{~nm}$ as a candidate for substrate texture.

A second length scale motivated by the suggested importance of the three-phase contact line, arises naturally from the notion that the contact line is characterized by a free energy per unit length, the line tension $\tau$. For a system involving air, supercooled liquid, nucleated solid, and catalyst substrate, four distinct line tensions exist and may play a role $[54,16]$. Regardless of which $\tau$ or combination of $\tau$ 's plays a role, the ratio of line and surface tension $\delta \sim \tau / \sigma$ suggests a length scale, below which free energy of the contact line exceeds free energy of the interface[14]. The existence or significance of the line tension itself is still a matter of some debate[79, 62], with conflicting reports in literature for the magnitude and even the sign[49,5]. Despite poor experimental quantification, however, recent computational[1] and nucleation studies[35] have reconciled observations to theory 


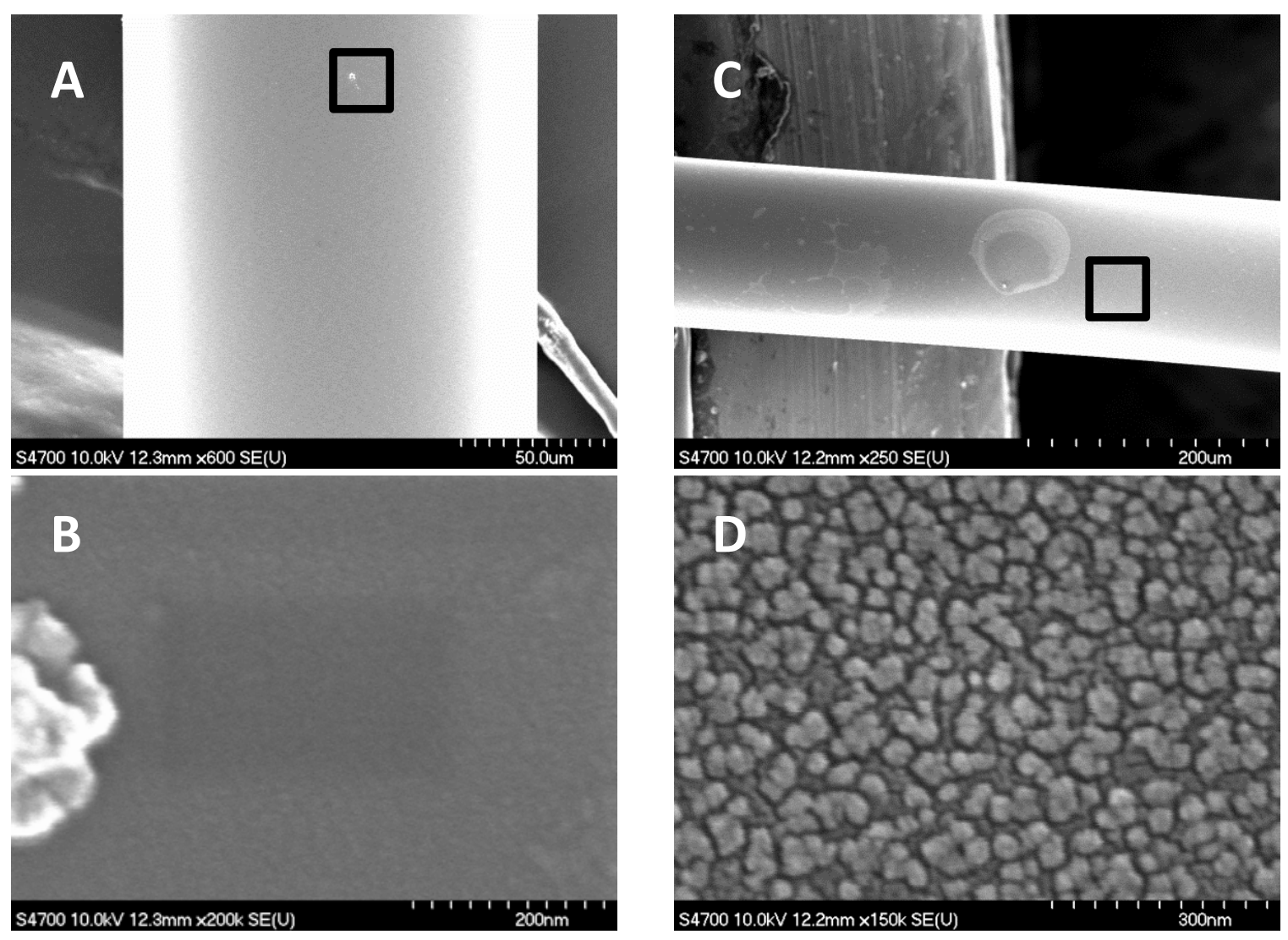

Figure 5.1: Fabricating nanoscale surface texture. Motivated by the conjectured importance of roughness to heterogeneous nucleation and the plausible range of length scales, these experiments were conducted with smooth optical fibers (A,B), nano-textured optical fibers (C,D), and micro-textured silicon substrates (see supplemental Figure 5.5) as heterogeneous nucleation catalysts. Panels A-D were taken with a high resolution SEM.

by including line tension. We ask, therefore, what substrate 'texture' length scales would introduce geometric variability to the contact line e.g., distortion due to pinning[14], that may affect the nucleation rate? Perhaps surface texture length scales on the order of or smaller than $\delta$ will promote surface nucleation? While $\sigma$ varies only slightly[14] with $T$, from $10^{-2}-10^{-1} \mathrm{~J} \mathrm{~m}^{-2}$, the reported range of values for $\tau$ is wide, from $10^{-11}$ to $10^{-8} \mathrm{~J}$ $\mathrm{m}^{-14}$, yielding a range of $\delta$ from $<1 \mathrm{~nm}$ to $\sim 1 \mu \mathrm{m}$.

${ }^{4}$ Values as large as $10^{-6} \mathrm{~J} \mathrm{~m}^{-1}$ have been reported (see references in [49]), which would correspond to
a length scale $\delta \sim 100 \mu \mathrm{m}$. Such large values have been questioned [14] and recent values seem to be 
In summary, length scales that could contribute to contact-line nucleation range from from the mm-scale of the macroscopic droplets for which no contact-line nucleation was observed, through the plausible range of $\delta$ starting at $\sim 1 \mu \mathrm{m}$, and finally down to length scales of minimum $\delta$ and the critical nucleus size $1-10 \mathrm{~nm}$. To that end, we have conducted nucleation experiments in which we observe freezing of water with high-speed optical imaging to identify the spatial origin of nucleation with droplets in contact with surfaces that are textured over a range of length scales. A shift to preference for nucleation at the contact line in these experiments would suggest that, whatever the physical mechanism, catalyst geometry plays a defining role. It then opens the way for further investigation of specific mechanisms using other methods, e.g., whether steps or pores resonant with the critical nucleus size, or distortion and curvature of the contact line on the order of $\delta$ lead to reduced Gibbs free energy barrier for nucleation.

Guided by the cascade of scales described above, these experiments were conducted with heterogeneous nucleation catalysts textured to exhibit specific length scales. The fabricated catalysts consist of micro-textured silicon substrates (see supplemental Figure 5.5), and nano-textured optical fibers (Figure 5.1, panels C and D). Untextured substrates and fibers were used as controls; a smooth fiber is shown in Figure 5.1 (panels A and B) for reference. To impose micrometer-scales, single-crystal silicon substrates with periodic patterns of linear surface features were fabricated using photolithography (see supplemental material). The spatial feature sizes explored were 50, 10, 5, and $2 \mu \mathrm{m}$; For large etch depths a superimposed, random texture with lengths in the range $1 \mu \mathrm{m}$ to $\sim 100$ $\mathrm{nm}$ also appeared (Supplemental Figure 5.5, bottom panel). To explore the nano-scales, below the limits of the photolithography method, an etching method was used on silica converging on the range $10^{-11}-10^{-8} \mathrm{~J} \mathrm{~m}^{-1}[75,60,35,5]$. 
glass fibers. Fibers without and with the resulting nanotexture are shown at two resolutions in scanning electron microscope (SEM) images (Figure 5.1 A-D). Image analysis of the texture shown in Figure 5.1 (D) reveals linear sizes from approximately $100 \mathrm{~nm}$ down to $2 \mathrm{~nm}$, which is near the resolution limit of the imaging method (see supplemental materials, section 4). Even in the absence of a contact line effect that changes with texture length scale, we can expect that roughness leads to an increase in catalyst surface area and therefore an increase in the extensive nucleation rate. The increases in surface area are small, but more importantly, the measurement depends on the spatial distribution of nucleation events, and is therefore is not directly dependent on quantification of nucleation rate.

The freezing of supercooled water droplets in contact with a catalyst is observed with a high speed camera at $200 \mu$ s between frames. The droplet is cooled at a rate of $2 \mathrm{~K} \mathrm{~min} \mathrm{~m}^{-1}$ to a temperature below the droplet freezing temperature $\left(T_{\text {Freeze }}\right)$, then warmed to $10{ }^{\circ} \mathrm{C}$ to melt the droplet, see supplemental information for more details. As shown in Figure 5.2, reversing the freezing process in time pinpoints the epicenter of crystallization. The process is repeated many times so that the spatial distribution of nucleation events can be measured. In each cycle the water droplet is cooled until freezing occurs and then heated and melted. The droplet rests on a substrate, as shown schematically in Figure 5.2. Looking from above, it is apparent whether there is a preference for nucleation events at the clearly visible three-phase contact line. For smooth substrates it has been confirmed[31, 32] that nucleation events are distributed randomly with no spatial correlations or preference for the contact line. When glass fibers are examined, the fiber pierces the drop as shown in Figure 5.2. Examples of nucleation events initiated on the substrate (red), on the immersed fiber (green), and at the fiber contact line (blue) are illustrated. Because the substrate 
and the fiber have essentially the same chemical composition (silica), a spatial shift from the substrate to the immersed fiber or to the fiber contact line is considered evidence for a change in the nucleation efficiency of those regions that represent a negligibly small fraction of the total catalyst surface area.

The microfabricated catalyst substrates, with length scales down to $2 \mu \mathrm{m}$ for the imposed pattern, and down to $100 \mathrm{~nm}$ for the deep etches, showed no change in the spatial distribution of nucleation events. Similarly, when the glass fiber with radius of $70 \mu \mathrm{m}$ was present, there was no tendency for nucleation to prefer the fiber over the substrate (see Figure 5.3, left panel). The nano-textured fiber, however displayed a shift in the spatial distribution of nucleation events to the fiber contact line. Despite the relatively small surface area of the nano-textured fiber, over half of the freezing events initiated there. And strikingly, despite the overwhelmingly small spatial odds, the majority of the fiber induced events originated at the three-phase contact line. This shift is consistent both with the surface texture length scale approaching the most likely value of $\delta \sim 10 \mathrm{~nm}$, and the length scale associated with the critical radius for nucleation $\lambda \sim 10 \mathrm{~nm}$. Of course, the spatial evidence alone cannot be considered direct evidence for one length versus the other. Regardless of motivation, the observation clearly supports the notion that nano-scale surface features strongly favor ice nucleation at three-phase contact lines.

In addition to the direct spatial evidence inherent to the design of the experiment, the temperature at which crystallization is initiated is also recorded, providing information on the efficiency of nucleation. Figure 5.3 (right panel) shows cumulative freezing probabilities versus $\Delta T$. Nucleation events at the fiber contact line (red curve) show significantly higher freezing temperatures, and these weaker supercoolings are indicative 


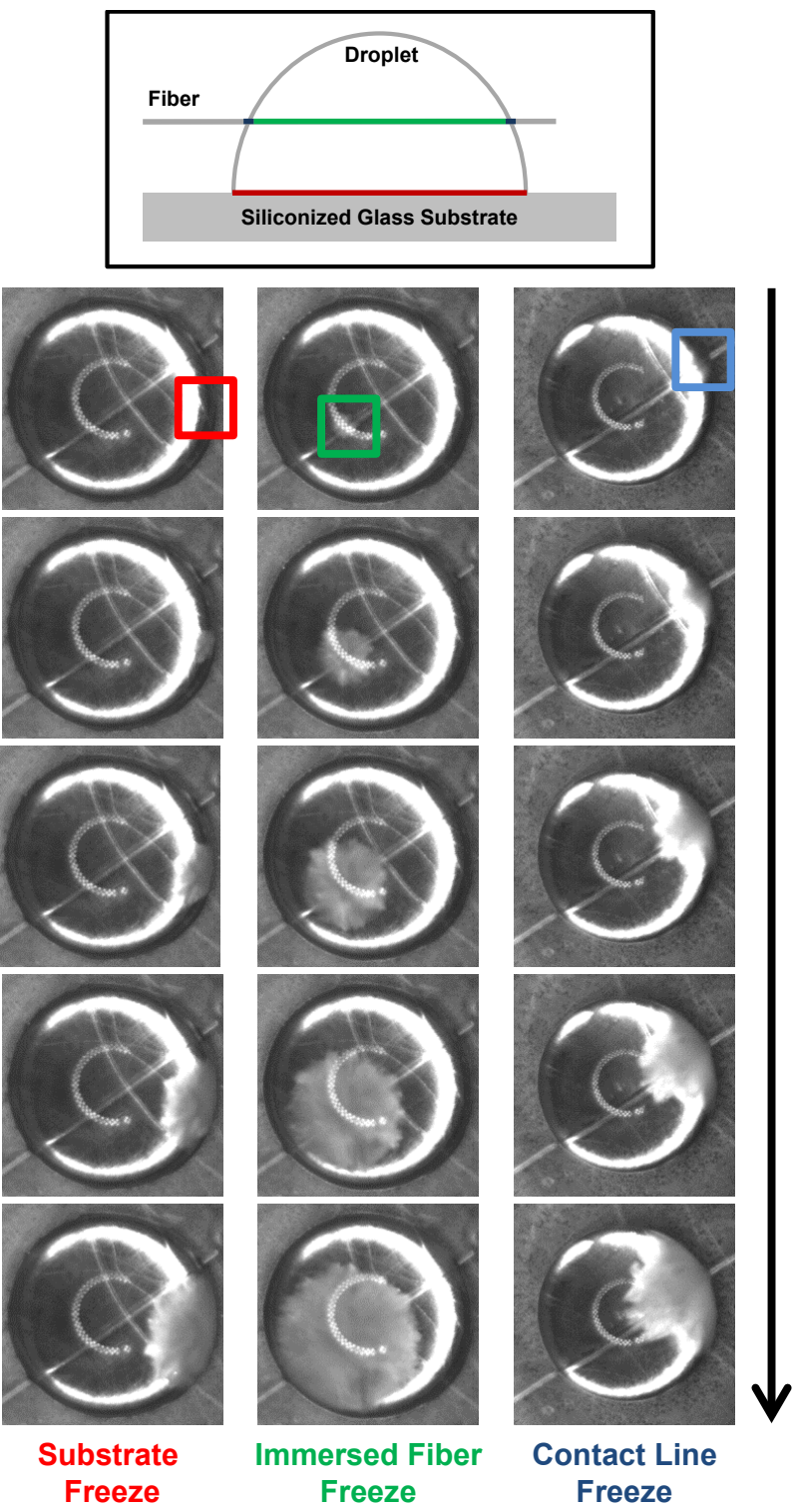

Figure 5.2: Three modes of nucleation. Top: A schematic of the droplet-fiber geometry. A $30 \mu \mathrm{L}$ droplet with a contact angle of $\approx 90^{\circ}$ rests on a siliconized glass slide (Hamilton Scientific) that is cooled from below [32]. An optical fiber, partially immersed within the droplet, can act as a heterogeneous nucleation catalyst. Three possibilities for nucleation then arise: on the substrate (red), on the immersed fiber (green) and at the fiber contact lines (blue). Bottom: By imaging the crystallization at $5 \mathrm{kHz}$ we pinpoint the nucleation site (boxed area in film strips). Film strips here represent each of the three nucleation modes. Every 15 th frame is shown resulting in a $3 \mathrm{~ms}$ spacing. 

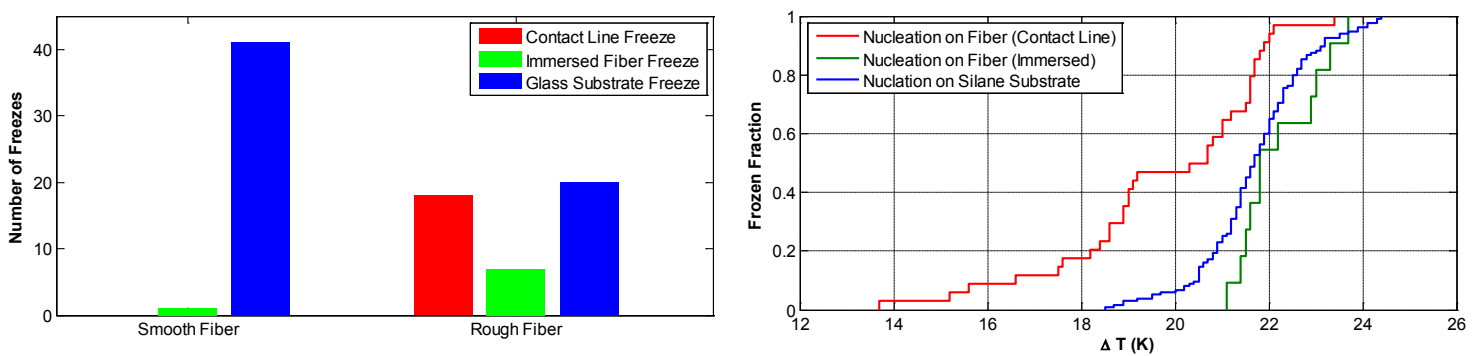

Figure 5.3: Nano-Texture observed to cause a transition to surface nucleation at the contact line. Results for the three modes of nucleation, substrate, immersed fiber, fiber contact line (see Figure 5.2). Left Panel: Spatial origin of crystallization is observed often to shift to the contact line for nano-textured (rough) fibers, but neither the smooth fiber $(r=70$ $\mu \mathrm{m})$ nor the micro-textured substrates $(2-100 \mu \mathrm{m})$ yield such a shift (see supplemental material, section 2). Despite relatively small surface area of the nano-textured fiber, over half of freezing events are initiated there. Furthermore, despite the overwhelmingly small spatial odds, the majority of the fiber induced events originate at the contact line. Right Panel: Higher freezing temperatures (weaker supercooling) are observed for nucleation events at the fiber contact line as evidenced by the cumulative freezing probabilities (red curve). Broadening of the distribution accompanies fiber contact line events, as expected because of additional variability in the geometry of the nano-textured contact lines.

of nucleation rates enhanced by many orders of magnitude[68]. The freezing temperature distribution for contact line events is not only shifted to higher temperatures, but is broadened. This is consistent with expectations for surface variability[67] because variance adds for independent causes (e.g., randomness inherent to nucleation and randomness associated with surface texture). The temperature distributions thus support the spatial evidence for surface texture inducing the change in freezing behavior.

Is the observed temperature shift $(\delta T \approx 3 K)$ consistent with the proposed mechanisms? Previous observations of contact line nucleation of ice suggest similar shifts of $\delta T=$ $2-5 K[19,23]$. It is customary in CNT to represent the efficiency of a heterogeneous catalyst through the contact angle $\theta_{o}$ of the nucleated phase, assumed to have the shape 
of a spherical cap: smaller contact angle implies more efficient catalyst. By comparison, to achieve $\delta T=1 K$ a $\Delta \theta_{o}=2^{o}$ is required ${ }^{5}$ [20]. Although line tension values for ice and water are poorly known, direct measurements of other substances, via the modified Young's equation $\cos \theta_{r}=\cos \theta_{o}-\tau / \sigma r$, show that $r \sim 10-100 \mathrm{~nm}$ droplets exhibit $\Delta \theta \sim 10^{\circ}[5,60]$. These values are consistent with our observed $\delta T$. Could nanopores explain this phenomenon? The nucleation barrier for $r_{\text {pore }} \approx r^{*}$ has been shown to be a half of that for a flat catalyst. CNT for ice in water can realistically result in $\delta T \sim 1-10 K$ for similar changes in $\Delta G$. However, this "pore-enhancement" can explain the magnitude of our observed temperature shift, but how they would cause a spatial transition to freezing at the contact line is unclear.

This evidence for a significant role of surface texture and characteristic length scales has wide implications: from catalyst design for drug synthesis, to improved parametrization of ice nucleation in clouds within weather and climate models. The demonstrated improvement in nucleation efficiency for nano-texture substrates is qualitatively consistent with recent work indicating that nucleation is enhanced by the introduction of sharp corners compared to circular shapes in catalysts with nano-pores[15, 65]. The relevant length scale may be the radius of curvature of a wetted surface feature, which is much smaller for 'square' nano-pores and is therefore consistent with the line tension hypothesis. The results from this work also help clarify why past work with 'point-like' contact[68] showed a strong preference for surface nucleation: It is likely that the naturally occurring, irregular, micron-scale particles used there have surface features on the order of or smaller than the line tension scale, $r<\delta$. This leads naturally to the speculation that spatially localized

\footnotetext{
${ }^{5}$ Similarly, an increase in surface area $\Delta A$ by approximately two orders of magnitude would be required to explain $\delta T=1 K$ [20]. The surface area enhancement between our smooth and textured fibers is estimated to be less than a factor of two $A_{\text {texture }}<2 A_{\text {smooth }}$, whereas our observed temperature shift would require an increase in surface area of 6 orders of magnitude compared to the smooth catalyst.
} 
regions that are thought to induce crystallization, known as 'active sites', may be associated with surface features (steps, kink sites, defects) with characteristic length scales at or below $\delta$.

\subsection{Acknowledgement}

This research was supported in part by an award from the Department of Energy (DOE) Office of Science Graduate Fellowship Program, the DOE Atmospheric System Research program through grant DE-SC0006949, and by the NSF grant AGS-111916. The

authors wish to thank Dr. P. Bergstrom and the Microfabrication Facility at MTU for assistance with substrate lithography, and also Dr. J. Drelich and the Applied Chemical and Morphological Analysis Laboratory at MTU for assistance with AFM and SEM measurements.

\subsection{Methods}

\subsubsection{Thermal Control Method}

We utilize the thermal control chamber described previously[32] to actively cool a water droplet resting on a substrate. Individual $30 \mu \mathrm{L}$ droplets were repeatedly frozen and melted (10-20 times) with a linear cooling rate of $2 \mathrm{~K} \mathrm{~min}^{-1}$. We cool the droplets continually from $10^{\circ} \mathrm{C}$ to $-25^{\circ} \mathrm{C}$ at $2 \mathrm{~K} / \mathrm{min}$, at which point the droplet is heated to $10^{\circ} \mathrm{C}$ to melt. The cycle is then repeated automatically until evaporating of the droplet, over many hours, 
renders the droplet too small to resolve and the cycle is manually stopped. The temperature and video of the freeze event are monitored and recorded by software that does not communicate with the thermal control PID system. A temperature difference develops within the cooling droplet[31], determined by the system cooling rate $\lambda$, droplet radius $r$ and thermal diffusivity $\chi$, as $\Delta T \approx \lambda r^{2} / \chi$, up to $2 \mathrm{~K}$. Thermal simulations[32] confirm that a constant vertical thermal gradient develops during cooling but horizontal gradients are minimal within the drop, so as to not bias freezing toward a particular region of the substrate or fiber. The temperature of the water droplet is taken to be that of the actively cooled copper heat sink below the substrate which is continuously monitored with an RTD probe $2.5 \mathrm{~cm}$ from the droplet. The remote thermal observation may introduce a systematic bias in the freezing temperature, but any bias will be constant across all droplets. The system geometry and cooling method is identical for both the substrate and fiber experiments.

Some variability in freezing temperature is observed between fibers. This variability is smaller than that observed for the shift from immersed to contact-line freezing, and freezing temperatures for the substrates closely resemble Gaussian distribution similar to those observed in our earlier work $[31,32]$. Because of the limited number of fibers, and the small number of freezing events per fiber, the variability of freezing temperatures between fibers is within the uncertainty from sampling of the Gaussian distributions. 


\subsubsection{Spatial Identification of the Nucleation Site and Results for Micro-Texture}

High speed imaging is used for resolving the spatial origin of nucleation sites, as described in detail elsewhere[31,32]. Using a vertically mounted high speed camera (Photron SA6) and an LED lighting array, we are able to trace the progression of the droplet crystallization (Figure 5.2). AVI movies recorded by the camera are examined frame by frame with ImageJ software to determine the nucleation site. With the micro-textured substrates we defined surface nucleation as beginning at the droplet triple line. For the nano-textured fiber study we similarly define surface nucleation as beginning where the fiber enters or exits the droplet. This approach is unique because it allows direct spatial evidence for the crystallization origin and geometry to be obtained, while still maintaining the ability to resolve shifts in freezing temperature. We note that in Figure 5.3 of the main text, thermal data are included even for some events for which the imaging system did not trigger properly (e.g., false triggers or camera failure). This is the same technique that we utilized in our previous work[32].

The possible influence of micro-texture on contact-line freezing is evaluated using a similar procedure as outlined in our prior work [31, 32]. We defined nucleation at the contact line to include any freezing sites that occur in the vicinity of the droplet perimeter (outermost $10 \%$ by area). Here the mean droplet size is $\approx 10 \mathrm{~mm}$ so we will consider any nucleation sites that occur within $0.25 \mathrm{~mm}$ of the contact line to have originated in the contact mode region (Figure 5.4 left panel). Assuming nucleation on the immersed substrate is a stochastic process and proportional to surface area, we would expect the 

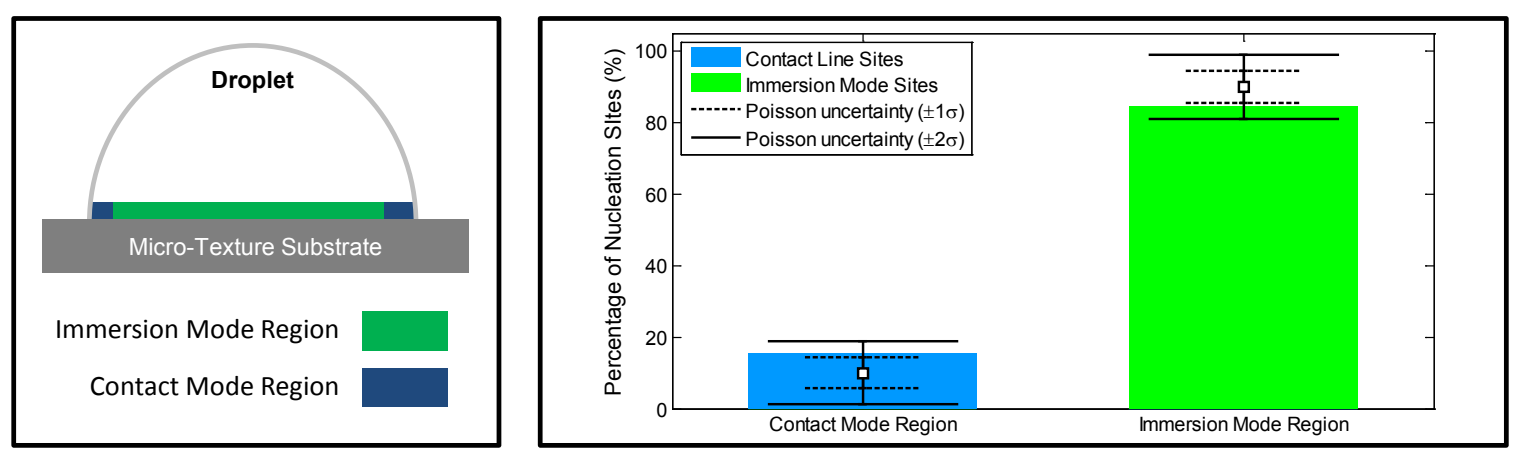

Figure 5.4: A comparison of the distribution of nucleation sites on the micro-textured substrates. Here, we identify the nucleation site at the substrate-water interface in the same way previously described (Figure 5.2 in article). Using the procedure outlined previously [31, 32] we classify any nucleation site located near the droplet perimeter, or outermost $10 \%$ of substrate-water interface region, as a contact mode freeze (Left Panel). When we sort the observed nucleation sites into Immersion and Contact freezes (Right Panel), it is clear that the number of sites is proportional to the surface area (observed number slightly exceeds $\pm 1 \sigma$ and is well within $\pm 2 \sigma$, where $\sigma^{2}$ is the Poisson variance), which agrees with our previous observations for smooth substrates [31, 32] and the stochastic assumption of classical nucleation theory. This result suggests that there is no enhancement for surface crystallization in the presence of micro-texture surface features.

number of nucleation sites in any given region of the substrate to be proportional to the immersed surface area. By this reasoning we would expect to observe $\approx 10 \%$ of the nucleation sites in the contact mode region. After image analysis of the micro-texture data we find that $\approx 15 \%$ of nucleation sites occur in the vicinity of the contact line, while $\approx 85 \%$ occur in the immersion mode (see Figure 5.4). This result lies within the Poisson variance of our data so there is no statistically significant enhancement in nucleation at the three phase interface. This null result stands in stark contrast to our result with nano-textured fibers, where $>50 \%$ of the nucleation sites on the fiber occur at the three phase interface (as described in the main text and Figure 5.3). 


\subsubsection{Fabrication of Micro-Texture Features}

Nucleation catalysts with micrometer-scale surface features are achieved through fabrication of square wave features on uniform silicon substrates similar to those used in previous work $[31,32]$. To fabricate repeated arrays of these structures we utilized a photolithography method to transfer a pattern (four $25 \mathrm{~mm}^{2}$ arrays of 4, 10, 20 and $100 \mu \mathrm{m}$ period square waves) from a laser etched photomask onto $100 \mathrm{~mm}$ prime grade $\langle 100\rangle$ silicon wafers. The wafers were first cleaned with a copious amount of acetone and isopropyl alcohol, then spin-coated with a layer of photoresist polymer, and finally placed in contact with the photomask and exposed with a UV lamp. Unexposed photoresist was then removed using RD6 solution and the wafer was washed and dried with a nitrogen spray. To etch the features into the silicon we employ a Reactive Ion Etch technique, where the wafer is exposed to a $\mathrm{CF}_{4}, \mathrm{O}_{2}$ and $\mathrm{CHF}_{3}$ plasma in two minute intervals to etch the entire substrate vertically with an etch rate of $150 \mathrm{~nm} \mathrm{~min}^{-1}$. Finally, any remaining photoresist is stripped with acetone, leaving a smooth wafer with trenches. The wafer is diced to separate the four patterned regions for testing in our thermal chamber. We repeated this process to make substrates with feature depths from 300 to $900 \mathrm{~nm}$. We utilized an atomic force microscope (AFM) in the tapping mode to examine the surface profile of the patterned regions (Figure 5.5). This technique yielded micro-textured substrates that were chemically homogeneous, with surface features of uniform depth, while allowing us to examine surface texture length scales as small as $2 \mu \mathrm{m}$. We note that the smoothness of the unprocessed wafers is less than the $2 \mathrm{~nm}$ roughness that can be resolved, as expected for $\mathrm{Si}$ wafers containing a native oxide layer. 
In the deepest etches $($ depth $\approx 900 \mathrm{~nm})$ additional surface roughening was encountered (e.g., see bottom panel of Figure 5.5). The AFM profile reveals texturing with horizontal length scales in the range $100 \mathrm{~nm}<r<1 \mu \mathrm{m}$. This roughening was caused by prolonged exposure to the reactive ion plasma during the etch process. The presence of this texture on a subset of the micro-textured substrates did not alter the spatial distribution of nucleation sites (Figure 5.4) compared to a smooth substrate [31, 32]. This observation indicates that length scales down to $r \sim 100 \mathrm{~nm}$ are insufficient to cause a transition to nucleation at the contact line. It provides further motivation to examine the role of sub-100-nm features, and helps to place an upper bound on the length scale $\delta$ that may be responsible for surface nucleation.

\subsubsection{Fabrication of Nano-Texture Features}

The lack of observed transition to contact-line freezing at scales down to $\sim 100 \mathrm{~nm}$ motivated the development of a freezing catalyst with $<100 \mathrm{~nm}$ texture. The photo lithography techniques used in the wafer-based substrate fabrication are unable to produce features at the lower end of the relevant length scale. Various methods were tried for producing $\sim 10$-nm-scale texture on substrates over the $\sim 1 \mathrm{~cm}^{2}$ area needed for the freezing experiments. The choice of optical fiber was motivated by a desire to find a catalyst that was chemically similar to the silica substrates, and smooth at the nm-scale, to compare with the textured substrates. Serendipitously, we found that when removing the cladding, a subset of the optical fibers exhibited nano-scale surface texture in the desirable range without changing the chemical composition. 

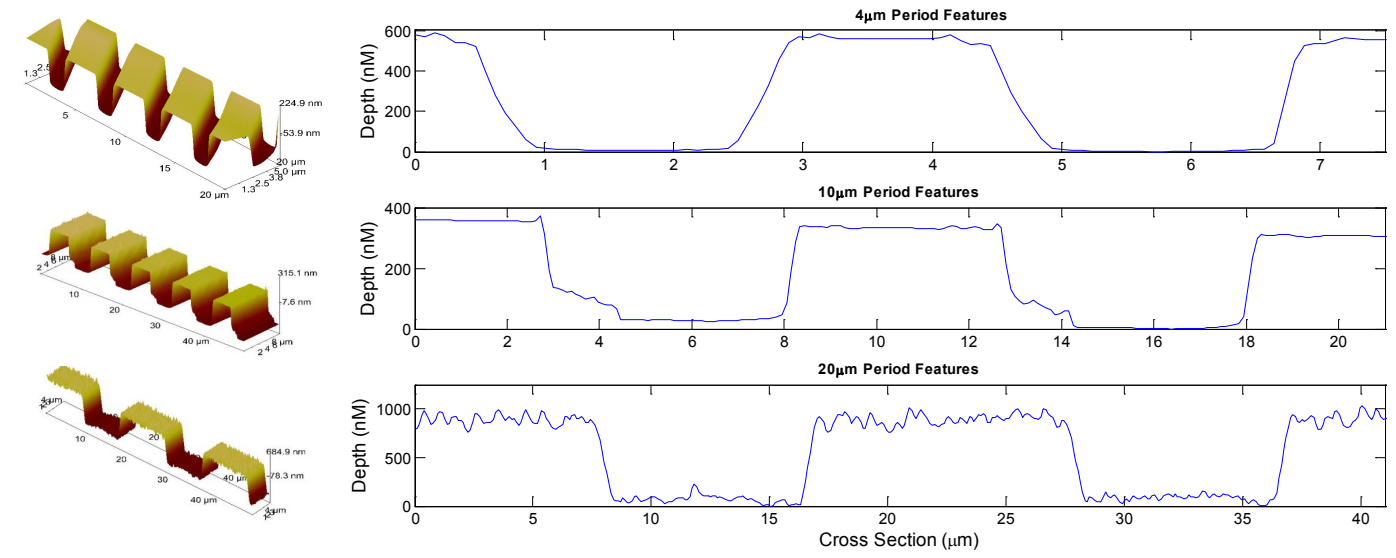

Figure 5.5: Fabricating surface texture from $100 \mu \mathrm{m}$ to $100 \mathrm{~nm}$. The profiles here indicate a subset of the micro fabricated substrates that we tested in these experiments. We utilize contact lithography with a reactive ion etch to create large $\left(2.5 \mathrm{~cm}^{2}\right)$ arrays of square wave features on homogeneous silicon substrates. We are able to control the period and depth of the structures to produce a uniform surface texture across the droplet-substrate interface. Here we show three dimensional (left) and two dimensional (right) Atomic Force Microscopy (AFM) surface profiles of a subset of our micro fabricated textured substrates. These substrates have square wave features with periods of $4 \mu \mathrm{m}$ (top), $10 \mu \mathrm{m}$ (middle) and 20 $\mu \mathrm{m}$ (bottom) with depths of $\approx 600,300$ and $900 \mathrm{~nm}$, respectively. Here we do not show AFM profiles for the largest period $(100 \mu \mathrm{m})$, because the scan range of the AFM is insufficient to observe multiple features. The deepest etches (depth $\approx 900 \mathrm{~nm}$ ) reveal additional texture in the $100 \mathrm{~nm}$ to $1 \mu \mathrm{m}$ range that result from prolonged exposure to the reactive ion plasma. The range of scales is therefore extended down to the 100 -nm scale.

Nucleation catalysts with surface features on the $\sim 10 \mathrm{~nm}$ scale are fabricated on optical fibers rather than substrates. The optical fibers are inserted into droplets in the thermal chamber using a piezoelectric linear stage, resulting in the geometry illustrated in Figure 5.2. In this geometry the droplet rests on a siliconized glass slide (Hampton Research HR8-088) that is more hydrophobic $\left(\theta_{\text {contact }} \sim 90^{\circ}\right)$ than silica $\left(\theta_{\text {contact }} \sim 60^{\circ}\right)$ and exhibits a lower freezing temperature. The optical fibers were an ideal candidate for their uniformity and similar chemistry to the native oxide surface layer of the silicon substrates. We utilise multi-mode fibers (Newport Optics F-MLD) with a silica core diameter of $140 \mu \mathrm{m}$, encased 
in a $250 \mu \mathrm{m}$ acrylate polymer coating. To expose the silica core, we remove the polymer sheath using two techniques, mechanical and chemical. In the mechanical process, the cladding is pulled off of the silica core after scoring with a razor. In the chemical process, the fiber is dipped in sulfuric acid to dissolve the polymer cladding. Examining the stripped fibers with a high resolution scanning electron microscope (SEM) revealed a wide variety of surface conditions, from featureless smooth fibers (Figure 5.1 A, B) to fibers saturated with small $(<100 \mathrm{~nm}$ scale, as defined by Diao et al.[15]) surface features (Figure 5.1 C, D) suitable for study of nano-textured nucleation catalysts. X-ray micro analysis of these surfaces confirmed the composition of both smooth and rough surfaces as purely silicon and oxygen, which implies that the surface texture is a result of the stripping technique. Although there was not a high consistency in fabricating the nanotextured surface patterns, possibly owing to the non-uniformity of fibers or sensitivity to subtle variations in the stripping processes, the resulting fibers ultimately are grouped in Figure 5.3A based on SEM imaging of their surface textures. So as not to bias our observations, the imaging was done after the fibers had been tested with droplets in the thermal chamber. Using these fibers we were able to compare nucleation behavior of catalysts with nano-texture (surface features $<100 \mathrm{~nm}$ ) to 'smooth' fibers with similar surface chemistry as in the studies on silicon substrates. Specifically, the un-etched fibers were found to be smooth down to the $\sim 2 \mathrm{~nm}$ roughness scale resulting from the coating process required for SEM imaging. The textured regions exhibited a relatively wide range of length scales even on single fibers.

We define a 'rough' or textured surface as one with a high density (approximately $>10$ features per $\mu \mathrm{m}^{2}$ ) of surface features $r<100 \mathrm{~nm}$ (see Figure 5.6). 'Smooth' fibers also occasionally contained some features in this size range, but at significantly lower surface density. The fabrication technique does not allow for finer segregation of surface texture 

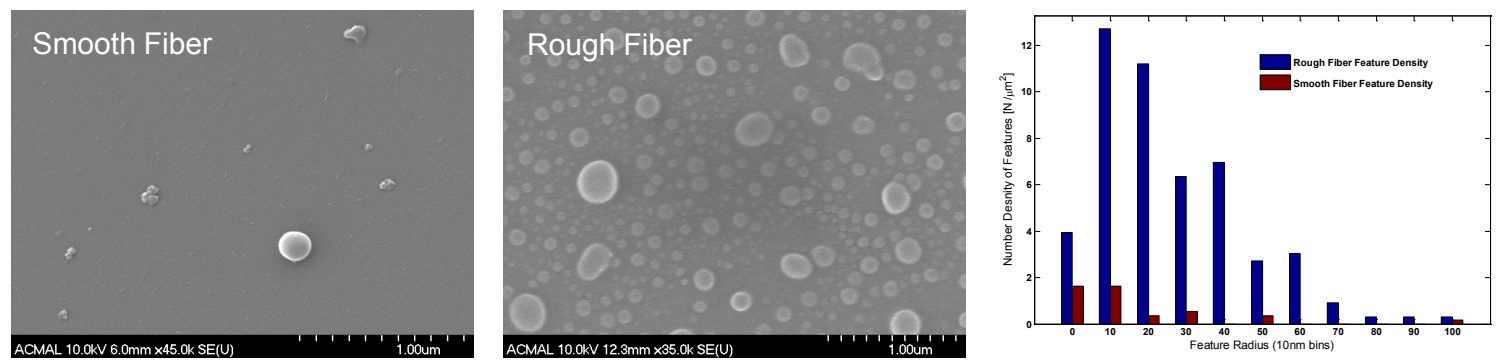

Figure 5.6: Surface morphology for representative smooth and rough fibers. Here the fibers (classified as smooth (left) and rough (middle) by our classification scheme) were imaged with under similar conditions and resolution with an SEM. The distribution of surface feature sizes, obtained with the ImageJ analysis tool, is indicated as number densities per area (right). For all fibers small scale features $(<10 \mathrm{~nm})$ exist but they are much more abundant on the rough fiber. It is possible that many of the smallest features may be anomalous artifacts from image processing, which would further reduce the density of the smallest features. This analysis excludes any features smaller than $2 \mathrm{~nm}$ because we have sputtered a 2-nm-thick coating of platinum to increase the conductivity of the sample, which was necessary for SEM imaging. It is unlikely that features below this size are relevant for ice nucleation, as they are smaller than the size of a typical critical ice nucleus.

length scales. Therefore, while this work provides the first direct evidence that $1-100$ nm length scales enhance surface nucleation, a more detailed analysis of length scales will require more refined surface texturing methods. 



\section{Chapter 6}

\section{Discussion}

In this chapter we review the results, including unpublished data, from Chapters 3-5 to highlight and summarize the key findings. We also discuss some open problems and make suggestions for future work, including some interesting things that we have observed along the way but have not thoroughly studied.

\subsection{Spatial Distributions of Nucleation Sites on Substrates}

Our experiments are unique in the ability to resolve not only a distribution of freezing temperatures, but also a spatial distribution of the nucleation sites. In our initial studies with macroscopic droplets resting on smooth substrates (Chapters 3 and 4), we observed no preference for nucleation to begin at the three phase contact line (summarized again in Figure 6.1). The distribution of freezing sites appeared to be distributed stochastically over the surface area of the substrate in agreement with classical nucleation theory under the 
assumption of uniform material properties. This result seems to be consistent over a broad range of droplet sizes $\left(5 \mu L<V_{\text {Drop }}<30 \mu L\right)$ and surface chemistries $\left(30^{\circ}<\theta_{\text {Drop }}<90^{\circ}\right)$, but it is important to note that for these studies the catalyst substrates we homogeneous used are atomically smooth.
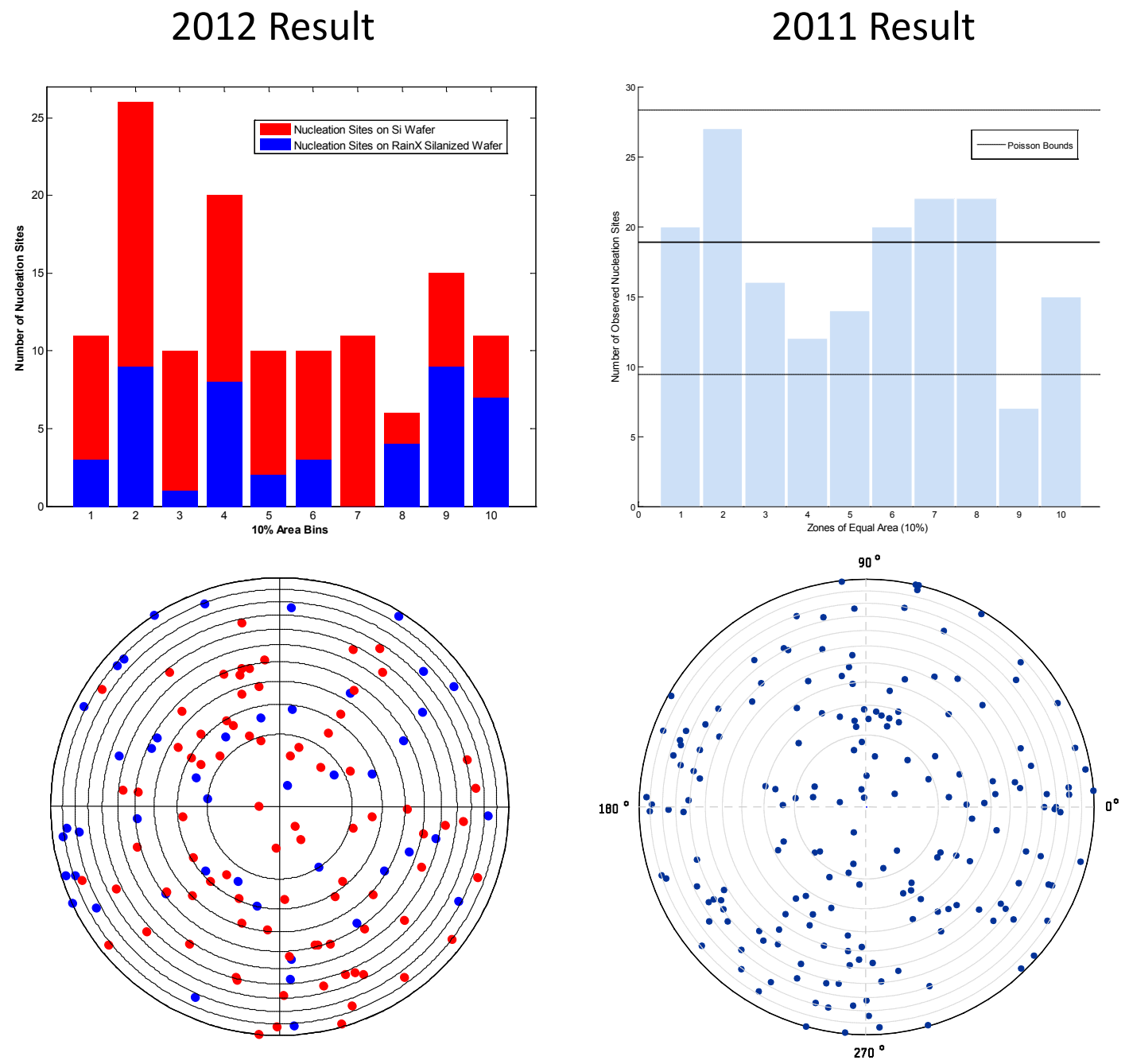

Figure 6.1: Droplet-Substrate Spatial Distribution Data Freezing sites are distributed uniformly over the droplet-substrate interface for data from Chapter 3 (right) and Chapter 4 (left). Here the data is derived from Figure 4.3 and Figure 3.4 
In our last study we introduce a system geometry with a catalyst fiber partially immersed in the a droplet. In this geometry, we simply classify freezing sites as originating from the fiber or underlying substrate. Examination of the fiber data reveals that freezing on the fiber only occurs (with one exception) when the fiber has been "roughened" to apply surface texture (Figure 5.3). When we isolate the fiber data we see that nucleation occurs preferentially at the fiber-droplet interface $\left(N_{\text {Contact }} \approx 3 * N_{\text {Immersion }}\right)$.

The distribution of freezing sites across the droplet-substrate interface directly contradicts an earlier study by Suzuki et al. [73], which reported freezing that always originates on the three phase contact line. This result is curious in that the same droplet-substrate is used as a nucleation catalyst. Our study in chapter 4 was designed in part to replicate the conditions of that experiment by incorporating larger droplets, stronger cooling rates and a horizontal high speed camera. Despite the increased cooling rates, we observed no change from the uniform spatial distribution from our first study (Figure 6.1).

In an attempt to reconcile our null result with the Suzuki et al. observations of contact freezing, we more closely examined the parameters of their experiment, namely the cooling technique and imaging technique. Thermal simulations of both systems indicate that a vertical gradient will develop within the droplet during cooling (which we argue will not bias nucleation (chapter 4)), but a high thermal conductivity of the system heat sink (copper used by Gurganus et al. and aluminum used by Suzuki et al.), will tend to minimize vertical gradients across the substrate. While there appears to be no thermodynamic explanation, a possible explanation does arise from the imaging technique. Suzuki et al. utilize a high speed camera to trace the evolution of the droplet crystallization, but critically, they rely on horizontal imaging. When we compared the images from our horizontal and vertical 


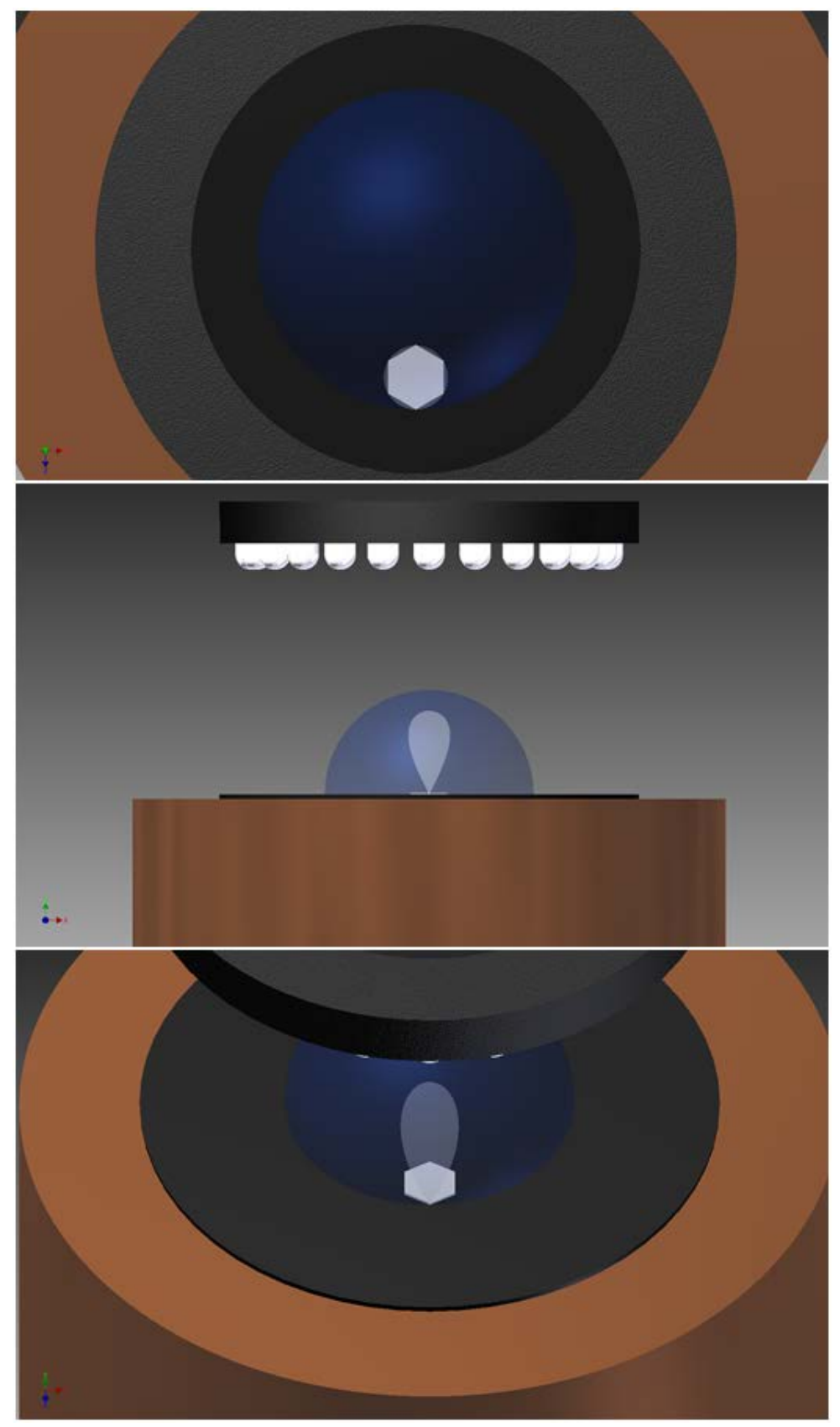

Figure 6.2: The effect of glint on the nucleation site detection Here, a hexagonal crystal grows from a nucleation site in the two-phase immersed region (away from the contact line). When viewed from the side it is possible that the projection of the crystal onto the curved droplet surface could be interpreted as freezing at the three phase contact line. 
cameras, we found that identifying the crystal nucleation site in the horizontal film was difficult due to a glint as the crystal grows. This glint originates from a internal reflection of the crystal, on the substrate, on the curved water air interface (Figure 6.2). When viewed from the side these spurious reflections create an appearance that the crystal is originating from the contact line.

\subsection{Freezing Temperature Distributions}

While the focus of our studies was the spatial distribution of nucleation sites, we also recorded freezing temperatures for all droplets. Classical nucleation theory assumes a that nucleation is a probabilistic process, defined by an approximately normally distributed freezing temperatures $\left(T_{\text {Freeze }}\right)$ for a constant cooling rate, indicated by a cumulative distribution function profile (CDF) for a droplet ensemble. In the cases of the simple droplet-substrate geometry presented here, this behavior is observed. The substrate freezing temperatures from Chapters 3-5 are all characterized by a normal distribution of freezing temperatures with clearly defined mean and variance (Figures 6.3, 6.4). The differences between mean freezing temperatures can be attributed to a variety of factors from different droplet sizes $(5-10 \mu \mathrm{L}$ in Chapter 3, to $30 \mu \mathrm{L}$ in Chapters $4-5)$, differences in temperature measurement techniques between chambers, different substrate materials (Silica, RainX and Siliconozed glass) and possible measurement bias from different cooling rates $(\lambda=0.1 \mathrm{~K} / \mathrm{min}-5 \mathrm{~K} / \mathrm{min})$. Because of the difficulty in directly comparing the freezing temperatures between the different studies, we will limit our discussion to data from individual experiments. 

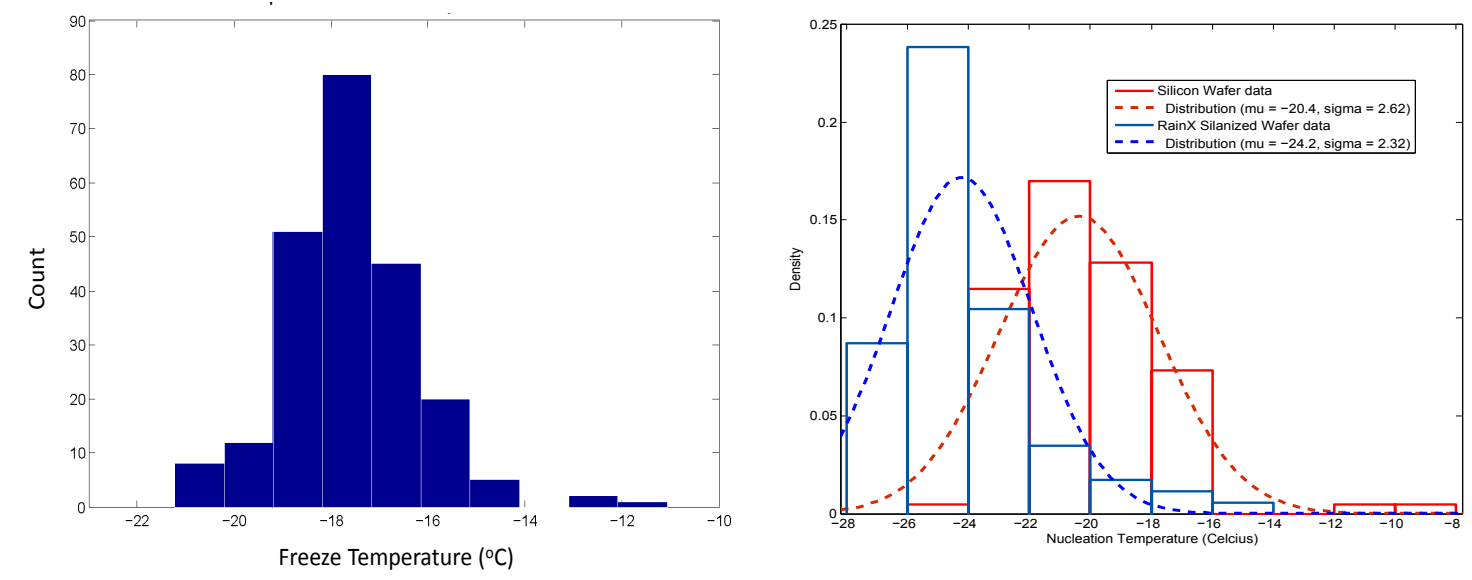

Figure 6.3: Freezing temperature $\left(T_{\text {Freeze }}\right)$ distributions from our two substrate studies In these studies (Left: Chapter 3, Right: Chapter 4), the temperatures are normally distributed with a clearly defined mean and variance. In our second study, a subset of the silicon wafers treated with RainX exhibit a much lower freezing temperature $(\Delta T \approx 4 K)$ but a very similar variance. This can be partly attributed to the reduction in surface area due to higher contact angle $\left(\Delta \theta_{\text {droplet }} \approx 30^{\circ}\right)$, but is mostly a result of the change in surface free energy $\left(\Delta \theta_{\text {icenucleus }}\right)$.

We will begin with a discussion of the simple droplet-substrate geometry of chapter 4 . In this study we compared freezing on a hydrophilic silicon and hydrophobic RainX silanized silicon wafer (Figures $6.3 \mathrm{~b}$ ). The shift in mean freezing temperature is clearly evident ( $\Delta T \approx 4 K)$, but perhaps more interesting is the very small difference in the variance of the distribution. The shift in freezing temperature cannot be attributed to a contact mode enhancement (see previous section), but can be partially explained by the reduction in the area of the droplet-substrate interface with increasing contact angle, because this area is an important variable in heterogeneous nucleation theory (Equation 1.2). Additionally, the RainX substrate is likely more icephobic than the silicon $\left(\theta_{\text {Ice,RainX }}>\theta_{\text {Ice }, S i}\right)$ resulting in a larger Gibbs barrier $\left(\Delta G^{*}\right)$ which would also tend to reduce the mean freezing temperature (Equation 1.11). A very small variance in the distribution is important, because it implies that the variance does not (strongly) depend on the contact angle of the droplet. This observation is important because it implies that the surface chemistry significantly changes 
the mean freezing temperature, but not the variance. It is important to note that this relation relies on the homogeneity of the atomically smooth silicon substrates used in this study.

In our last study (chapter 5) we incorporate non-homogeneous catalysts to examine the role of surface texture. As in the prior work, we again utilized a spherical cap droplet but with a different hydrophobic substrate. The distribution of freezing temperatures on these new substrates still exhibits a normal shape, with an even smaller temperature variance than that in chapter 4. From this distribution we can safely argue that the silconized glass wafers are homogeneous, like the silicon wafers. It is also important to note that the mean freezing temperature for the optical fiber immersion mode is nearly identical to that of the substrate $(\Delta T \approx 0)$. The glass fiber is composed of silica, which is similar to the oxide surface of the silicon wafers so we expect the fibers to have a correspondingly larger nucleation rate than the hydrophobic substrate $\left(J_{H e t, F i b e r}>J_{H e t, \text { Substrate }}\right)$, which should result in a warmer freezing temperatures based on prior observations ((Figures $6.3 \mathrm{~b})$. In that study the more hydrophilic substrate had a larger droplet-catalyst surface area, while here the opposite is true. It is for this reason that in the case of the smooth homogeneous fiber inserted into the droplet, all of the nucleation sites (save one) can be traced to the hydrophobic substrate (Figure 5.3 a). This suggests that a significantly increased nucleation rate for hydrophilic substrates $\left(j_{\text {hydrophillic }}>j_{\text {hydrophobic }}\right)$, can still be offset by a large increase in surface area (Equation 1.2).

When we apply texture to the fibers, thereby increasing the total surface area of the catalyst $\left(A_{\text {rough }}>A_{\text {smooth }}\right)$, there is a small enhancement in freezing temperature observed for immersion mode freezing. More significantly however, is the appearance of contact freezing events at the droplet-fiber contact line. Fitting of a Gaussian distribution to the 


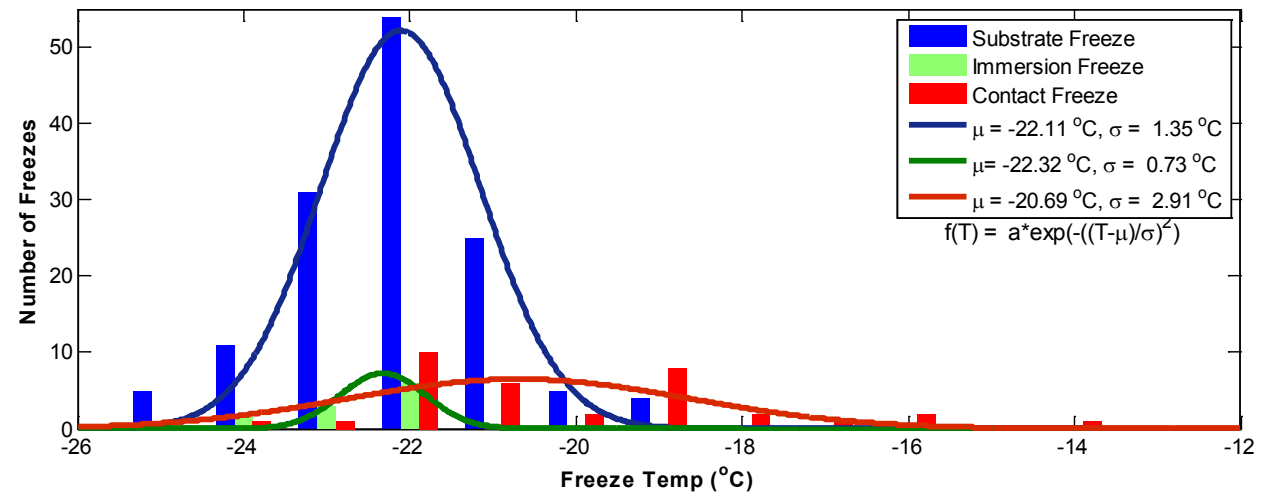

Figure 6.4: Freezing temperature $\left(T_{\text {Freeze }}\right)$ distributions from our surface texture study Freezing temperatures for crystallization originating at the substrate interface have a similar distribution to our earlier experiments (Figure 6.3). Similarly, the few occurrences of immersion freezing on the fiber have a very small variance. Nucleation events beginning on the fiber contact line, exhibit a very large variance. A shift in the mean temperature for contact and immersion freezes $(\Delta t \approx 2 K)$ is in line with earlier observation of contact nucleations $(\Delta T=2-5 K)[19,68,23]$.

contact freezing temperatures produces a higher mean freezing temperature $(\Delta T \approx 2 K)$ in line with other observations of contact freezing.[19, 68, 23] More importantly, the variance in the distribution increases significantly. This could be attributed to a general paucity of data, but this simple explanation is unlikely when we consider the distribution of even more sparse immersion data. The distribution of potential nucleation sites on the fibers (both in size and location) and the distribution of temperatures for a given site are independent random variables [67] whose variances are additive, and may contribute to the large variance in observed freezing temperatures $\left(\sigma_{T}\right)$. This large variance, as well as the continued existence of substrate freezing events and immersion events is then likely attributed to our inability to control the surface texture, both feature size and distribution. 


\subsection{The Importance of Surface Features}

Examination of the temperature and spatial data from these experiments indicates that the most important factor for contact line nucleation is catalyst surface texture. In each case the smooth catalysts (substrates and smooth fibers) show no preference for nucleation at the three phase interface. Surface texture or roughness is typically viewed as a macroscopic measure of the difference in surface area of between surfaces. In this regard, an enhancement in immersion nucleation from surface texture can be taken as an increase in the surface area of catalyst which will increase the probability of nucleation (increasing the mean freezing temperature) through Equation 1.2. Moreover, this enhancement cannot explain why nucleation would be preferred at the triple line. In the case of the fibers, the total immersed area of the fiber is several orders of magnitude greater than the area that might interact with the droplet interface (estimating the oscillations of the droplet that might cause the shift the contact line), yet the mean freezing temperature and total number of nucleation sites are greater for the contact mode.

Despite the higher freezing temperature of contact nucleation events, some substrate freezing events are observed when a "rough" fiber is inserted into the droplet. A possible explanation for these observations is found in our work with "roughened" substrates, described in Chapter 5. As we note in Chapter 1, the existence of line tension might be sufficient to lower the energy barrier for nucleation. In Chapter 5, we expand on this idea and suggest that line tension is only relevant for nucleation in the presence of a very small contact line, in the vicinity of very small catalyst features $\left(\delta<\frac{\tau}{\sigma}\right)$. These nano-scale variations in contact line can be formed directly due to geometry, or by the 
pinning of a receding macroscopic contact line to a small surface feature or defect (Figure 1.8). This requires the presence of these small features on a larger substrate (or particle) in the vicinity of the droplet contact line. Due to the wide range of values for $\tau$ in literature, we sought to examine surface features ranging from $r \approx 10 \mu m-10 \mathrm{~nm}$. To span this broad range, we conducted a series of experiments with micrometer-scale surface features and nanometer-scale surface features to identify the onset conditions for nucleation at the contact line.

To examine the role if micrometer scale features we used micro fabrication techniques to create large $\left(20 \mathrm{~cm}^{2}\right)$ areas of periodic square wave features on silicon wafers, with the periods from $4 \mu m-50 \mu m$ (Figure 5.5). Droplets were placed on these patterned regions so that the droplet-substrate interface and the droplet contact line were both in contact with micron scale surface features. Repeating the experiments described in chapter 4 with these new substrates yielded no change in spatial distribution from the smooth wafers. This result is highlighted in Figure 6.5, where a droplet is placed at the boundary between a patterned and "smooth" region of a silicon wafer. Over repeated freeze-thaw cycles, the nucleation site is observed to vary randomly between the patterned and smooth regions indicating no preference for nucleation in the micro textured region. This null result suggests that contact-line nucleation may only be relevant in the presence of smaller scale surface features $(r<2 \mu m)$.

To examine the role of nanometer scale features we used a sulfuric acid to etch glass fibers (Figure 5.1). The result from that study suggests a bound on the relevant surface texture length scale of $r_{\text {nanotexture }}<100 \mathrm{~nm}$ (Figure 2.8) for contact-line nucleation to be observed. If line tension accounts for these observations then the scaling factor $\delta$ (equation 


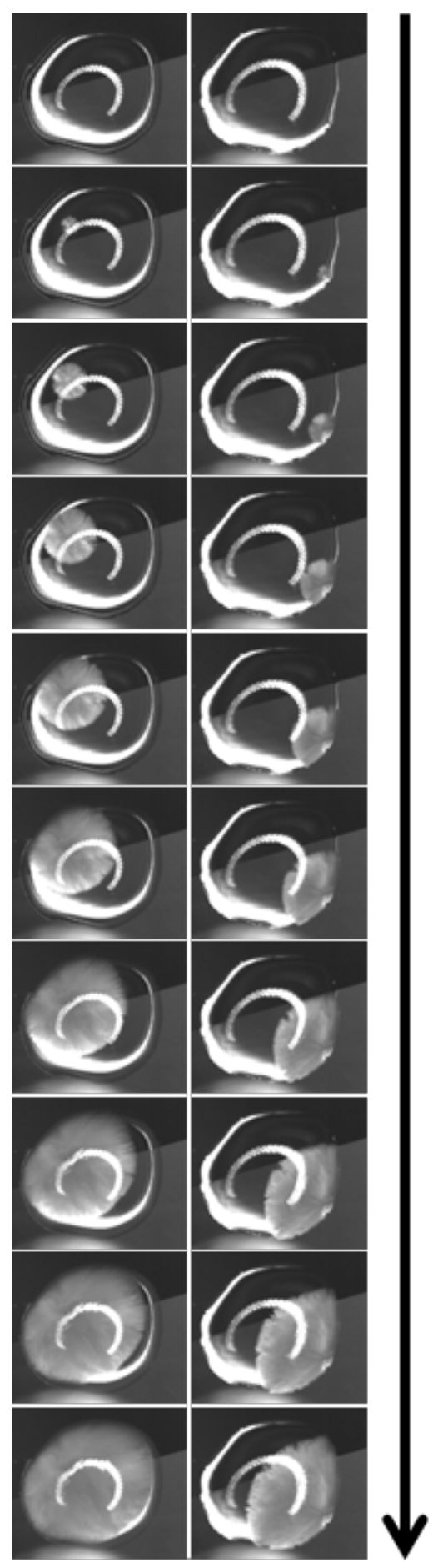

Figure 6.5: A droplet rests on two regions on a silicon substrate. The darker region of the substrate is smooth, while a micro surface texture (Figure 5.5) has been applied to the lighter region as described in Chapter 5. During repeated freeze-thaw cycles, crystallization was initiated on both regions, indicating no preference towards the micro textured region. 
1.27) must be of the same order as $r_{\text {nanotexture }}$. With a typical value for surface tension of $\sim 10^{-1} \mathrm{~J} / \mathrm{m}^{2}$, line tension would need to exist with in the range of of $\tau<10^{-8} \mathrm{~J} / \mathrm{m}$, which lies within the range of values reported in literature[50]. More modern estimates of $\tau$ place a lower bound at $\tau=10^{-10} \mathrm{~J} / \mathrm{m}$ [5], which would suggest a relevant surface feature size on $r_{\text {nanotexture }} \sim 1 \mathrm{~nm}$. A subset of features on the roughened fiber might contain length scales in this regime $(r<10 \mathrm{~nm}$, Figure 2.8).

\subsection{Conclusion}

The experiments described here were designed to examine nucleation at the three phase interface. By using simple droplet-substrate and droplet-fiber geometries, we were able to isolate and catalog spatial distributions of nucleation sites in an unprecedented way. We were also able to disentangle thermodynamic and chemical mechanisms by relying on a uniform catalyst material for all experiments (silica). These challenges highlight the complexities inherent to the study of nucleation at the contact line and its possible role in surface crystallization.

In the end, our data points toward the importance of surface texture for contact-line nucleation. There appears to be a relevant length scale, $r_{\text {nanotexture }}<100 \mathrm{~nm}$ for nucleation by surface features piercing or distorting the three phase contact line. The physical explanation of this phenomenon remains elusive, but a possible candidate can be found in the existence of a line tension energy at a three phase interface. Here we use this idea to develop a simple scaling factor $\delta$, which relates the counteracting roles of the line and surface energies. At length scales $r_{\text {nanotexture }} \sim \delta$ the barrier for nucleation at the three 
phase interface $\Delta G_{\text {surface }}$ is sufficiently lowered, which might explain a preference for contact nucleation for some particles. The relevance for this mechanism in the atmosphere is somewhat limited by a generally poor characterization of aerosol particles at these scales, as typical studies seek only to study the shape and composition of aerosols which are important properties for scattering. This work suggests that new studies are needed to map and characterize the surfaces of particles, to determine the relevance of point-like contact-line freezing from aerosol surface features smaller than $\delta$.

\subsection{Other observations and Future Work}

In this section we will discuss some of the other techniques we tried along with a number of intriguing and puzzling observations.

\subsubsection{Point-Like Contact}

In this work we conclude that the existence of nano-texture surface features is likely responsible for the contact nucleation. A broader question must be posed as to the importance of this finding on atmospheric systems. We attempted to find catalyst materials with sufficiently small surface features, but were continually frustrated. We began with atomically sharp scanning tunneling microscopy (STM) tips, but were unable to trigger nucleation by piercing droplets with these tips (Figure 6.6). Closer examination of these tips with an SEM reveals that they are often blunted wither by handling or by interaction with the droplet. In either case, we rarely observe surface features (and tip apexes) with diameters smaller than $100 \mathrm{~nm}$. The difficulties in producing a truly "sharp" probe 
$(r<100 \mathrm{~nm})$, and positioning it exactly at the droplet interface limit the effectiveness of this technique. In the future, contact studies should continue to focus on micro fabricating substrates to provide a large number of surface features in contact with the three phase interface.
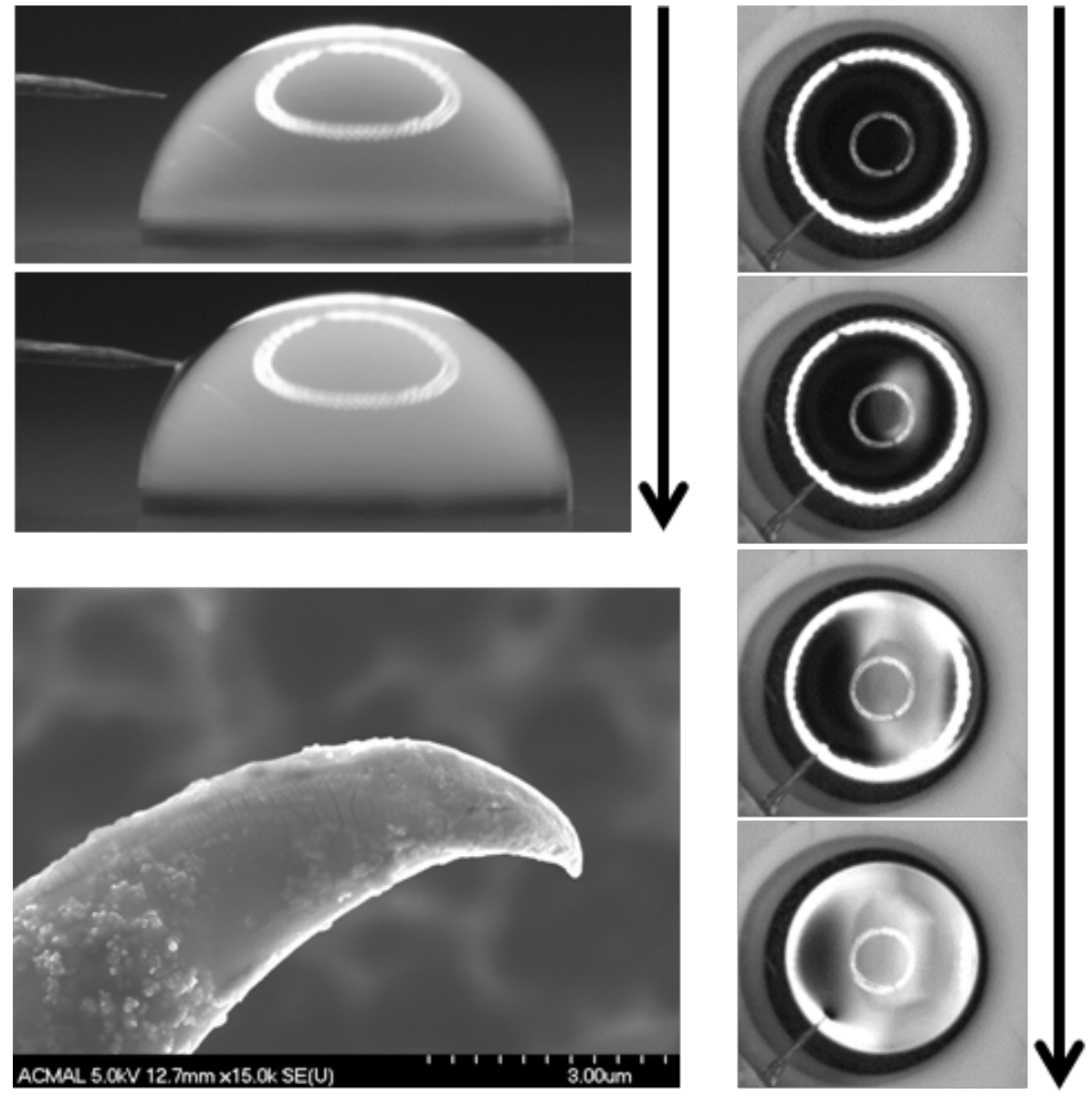

Figure 6.6: Poking the droplet Here an electro-chemically sharpened tungsten tip is brought into contact with a spherical cap droplet (top left). Nucleation is initiated on the hydrophobic substrate, away from the probe (Right). SEM analysis of the probe tip reveals a blunted tip and course surface features $(r>100 \mathrm{~nm})$. 


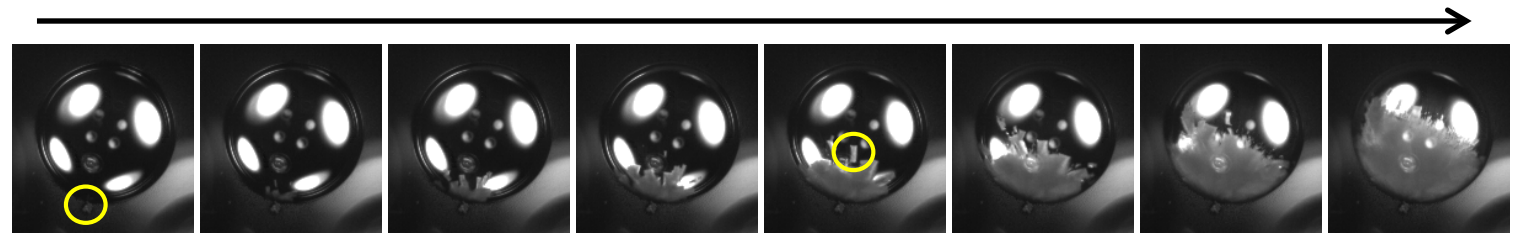

Figure 6.7: An example of nucleation occurring ahead of the crystallization front. Here nucleation is induced by an external crystal in a "Cooper mode" event (yellow circle in frame 1). Crystallization of the droplet proceeds from the nucleation site, but new bar shaped ice crystals appear to form ahead of the crystallization front (yellow circle frame 5). When viewed at full speed it is clear that a spherical wave, originating from the catalyst site, precedes the nucleation of these new crystals.

\subsubsection{Capillary Waves}

We have occasionally observed of spherical waves originating from the nucleation site. We are unable to determine if they are surface capillary waves or an glint caused by a change in the index or refraction with change in droplet temperature. In either case, we note that these spherical waves proceed faster than the droplet surface crystallization at warm freezing temperatures. More interestingly, we observe that new crystals can be nucleated behind this wave front, but ahead of the crystallization front. The mechanism responsible for these observations remains a mystery.

\subsubsection{Bubble Nucleation}

As part of our experiments with optical fibers in Chapter 5, we occasionally noted the presence of small bubbles in the supercooled droplets. The bubbles tended to form on the immersed fiber, and persist over several freeze thaw thermal cycles before dissipating. They might be formed on small air pockets on the roughened fibers growing as air bubbles 
conglomerate as the droplet thaws. Bubble formation is more likely tied to surface roughness of the fibers, with small surface features acting as catalyst sites for bubble formation, because they tend not to form in droplets resting on smooth substrates. The formation and dissipation of the bubbles is interesting, but here we will discuss their possible role in ice nucleation of the droplet.

During one fiber experiment, we isolated the nucleation site to the very center of the droplet (Figure 6.8) at $T_{d r o p} \approx-20^{\circ} \mathrm{C}$. During this freezing cycle (the 15th for this droplet) a cluster of bubbles, formed previously on the immersed fiber, were congregated at the apex of the droplet due to their buoyancy. While reviewing the video we observed several of the bubbles "burst," which immediately preceded the formation of an ice crystal in the same location. A mechanical shock front accompanied the bubble "pop," indicating a large energy dissipation. It appears that the bursting of the bubble created a thermodynamic condition where homogeneous nucleation could proceed away from the substrate and fiber catalysts.

To explain how the drop could freeze without a catalyst, yet above the homogeneous nucleation threshold $\left(T \approx-38^{\circ} C\right)$, we will focus on the conditions created by the bursting bubble. The energy associated with small collapsing bubbles can be large enough to produce photons in a process called sonoluminescence. Bubbles of lower density $\left(\rho_{\text {air }} \ll\right.$ $\rho_{\text {water }}$ ) can be maintained in a fluid because of a higher internal pressure. The pressure difference across the droplet interface $\Delta P$, the Laplace Pressure, is a function of the radius of curvature ( $\left.r_{\text {curvature }}\right)$ and and surface tension $(\sigma)$

$$
\Delta P=\frac{2 \sigma}{r_{\text {curvature }}}
$$




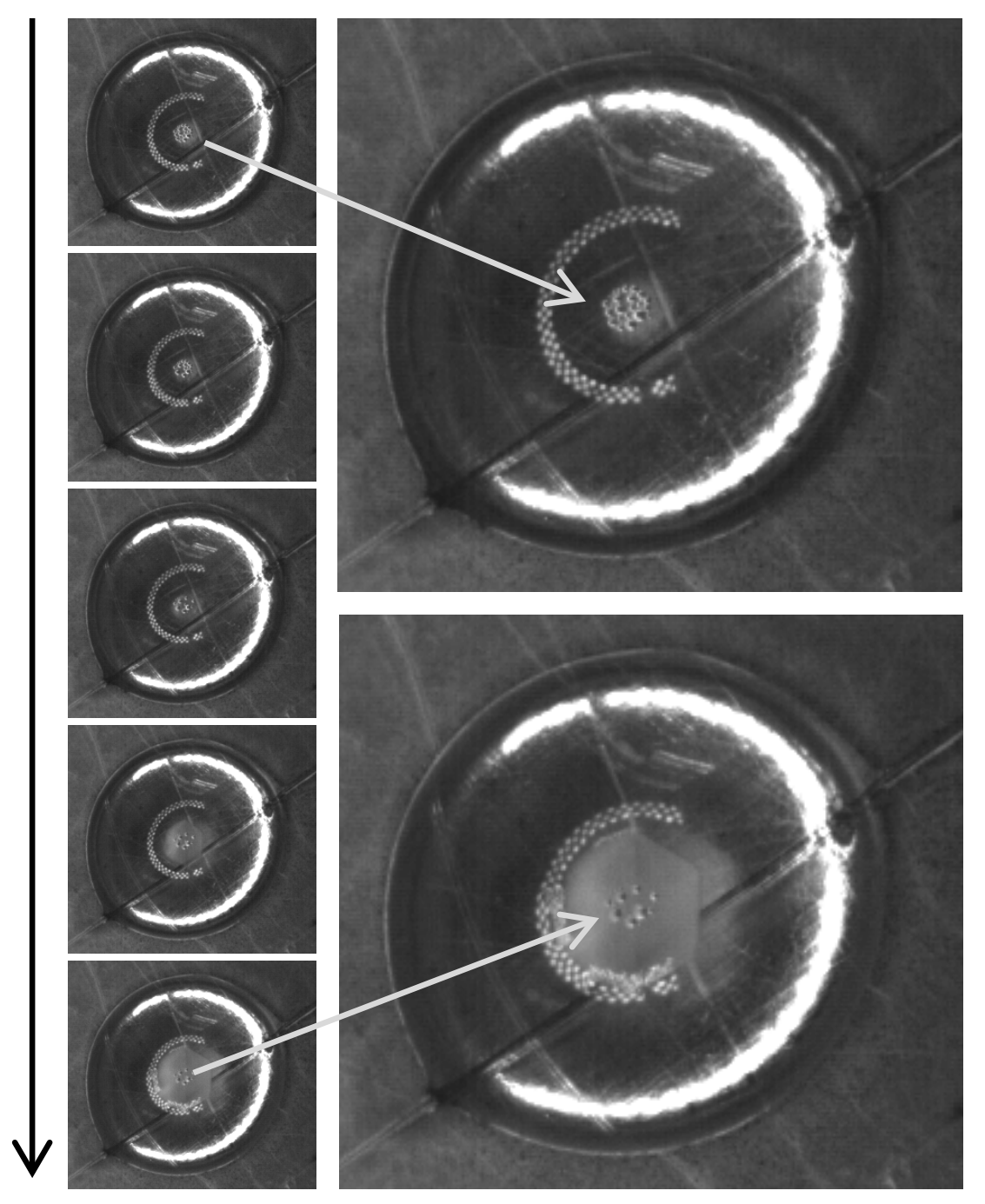

Figure 6.8: A rare example of nucleation induced by bursting of a bubble. A film strip shows the crystallization beginning at the top of a droplet, with several bubble trapped at the apex. A closer examination (right), reveals a decrease in the number of bubbles between before and after the formation of the crystal. When played in at full speed, it is clear that the nucleation is triggered by the bursting of one or more air bubbles.

Two mechanisms have been proposed to explain how the pressure wave associated with a collapsing cavity, or a popping bubble in this system, may lead to thermodynamic conditions for nucleation[34]. The first assumes a compression of supercooled water surrounding the droplet, and the second assumes an adiabatic expansion of the bubble. 
Both conditions are plausible in that we expect a strong overpressure proceeding the shock front and an under pressure to follow in its wake.

\section{Nucleation by Compression}

Perhaps the simpler of the two mechanisms, in the compression mode it is assumed that a small volume of supercooled water is compressed by the shock front. Under these conditions $\left(\Delta P \gg P_{\text {Ambient }}\right)$, nucleation in the hexagonal phase is impossible. However, nucleation of ice is not precluded at higher pressure phases[46], like Ice(VI) or Ice(VII), if $\Delta P>10^{5} A T M[34]$. In this scenario the critical ice nucleus would be formed, and grow as a metastable Ice phase. We would expect a relaxation to the stable Ice phase for the ambient temperature (Ice(I) hexagonal) to occur during via annealing during the second phase of droplet crystallization where the droplet is warmed to the melt temperature.

Unfortunately, given the approximate size of the bubbles we observe in Fig. 6.8 $\left(r_{\text {Bubble }} 10 \mu \mathrm{m}\right)$ we would only expect modest compression $\left(\triangle P \sim 10^{5} \mathrm{~Pa}\right.$ or $\left.\sim 1 \mathrm{ATM}\right)$, from the Laplace Pressure 6.1. This is well below the regime proposed by Hickling [34], so this mechanism is unlikely to be responsible for this observation.

\section{Nucleation by Expansion}

When a bubble bursts, the pressure in the volume equalizes rapidly to that of the fluid eliminating the pressure gradient. In this scenario, the air bubble expands rapidly whereby cooling water in its vicinity. We will assume that this process occurs sufficiently rapidly 
to be considered adiabatic, whereby the change in temperature is related the change in pressure

$$
\frac{T}{T_{o}}=\left(\frac{P}{P_{o}}\right)^{\frac{\gamma-1}{\gamma}}
$$

where $\gamma$ is the ratio of specific heats for water $\left(\gamma=\frac{c_{p}}{c_{v}}\right)$. It is easy to see that for air $(\gamma \approx$ 1.4)[27], this adiabatic expansion will result in cooling of the volume. From this expression we can develop a relation for a shift in temperature $\left(\Delta T_{\text {expansion }}\right)$ immediately following the bubble burst.

$$
\Delta T_{\text {expansion }}=T_{o}\left(\left(\frac{P}{P_{o}}\right)^{\frac{\gamma-1}{\gamma}}-1\right) .
$$

Substituting equation 6.1 in to equation 6.3 , we arrive at

$$
\Delta T_{\text {expansion }}=T_{o}\left(\left(\frac{2 \sigma}{r_{\text {curvature }} P_{o}}+1\right)^{\frac{\gamma-1}{\gamma}}-1\right)
$$

The bubbles in Figure 6.8 are smaller than the fiber $(r<70 \mu m)$ and would result in an instantaneous cooling of $\Delta T>160 \mathrm{~K}$, using values for supercooled water in this instance $\left(\gamma \approx 1.4, \sigma \approx 0.1 \frac{J}{m^{2}}, T_{o}=255 K, P_{o}=610.5 P a\right)[27]$. This strong cooling is sufficient to overcome the homogeneous nucleation threshold (at any supercooling temperature) and may be responsible for our observations. This mechanism in interesting, but as the bubbles only develop over repeated freeze-thaw cycles it remains unclear how relevant it is to atmospheric conditions. 


\subsubsection{The Role of Humidity}

I has been recently noted that humidity might play a role in surface crystallization. Jung et al. [41] observed shift between heterogeneous nucleation of a spherical cap droplet (at the substrate), and the homogeneous surface crystallization on the water-air interface, which they attribute to the role of evaporative cooling in a sub-saturated environment. In our studies, we were not able to control the exact relative humidity in the chamber. When using the chamber described in Chapters 4 and 5, the air chamber volume was not cooled uniformly with the substrate which resulted in large super saturations and vapor deposition onto the substrate. This is evident in the right column of Figure 6.6, where the opaque region around the droplet indicates frost formation.

To minimize frost formation for the actively cooled chamber in chapter 4 , an small overpressure was created in the chamber with a nitrogen flow. When this flow is reduced or removed, the humidity in the chamber tends to increase towards that of the laboratory ambient. For the chapter 5, we utilized hydrophobic substrates which were less prone to wetting allowing us to work at higher chamber relative humidities. In the presence of higher relative humidity, we tended to observe freezing preferentially at the droplet perimeter. Because substrate freezes were disregarded in that study, the distribution of substrate freezes was not examined in detail (as in chapters 3 and 4). The mechanism responsible for a shift to contact-line freezing at higher relative humidity remains a mystery. 


\section{References}

[1] S. Auer and D. Frenkel. Line tension controls wall-induced crystal nucleation in hard-sphere colloids. Physical Review Letters, 91(1), 2003.

[2] L. S. Bartell, Y. G. Chushak, and J. Huang. Structure and transformation in clusters: Computational experiments. Structural Chemistry, 11(2-3):105-110, 2000.

[3] S. Bauerecker, P. Ulbig, V. Buch, L. Vrbka, and P. Jungwirth. Monitoring ice nucleation in pure and salty water via high-speed imaging and computer simulations. Journal of Physical Chemistry C, 112(20):7631-7636, 2008.

[4] O. Benchikh, D. Fournier, A. C. Boccara, and J. Teixeira. Photothermal measurement of the thermal-conductivity of supercooled water. Journal de Physique, 46(5):727-731, 1985.

[5] J. K. Berg, C. M. Weber, and H. Riegler. Impact of negative line tension on the shape of nanometer-size sessile droplets. Physical Review Letters, 105(7):076103, 2010.

[6] C. F. Bohren and D. R. Huffman. Absorption and Scattering of Light by Small Particles. John Wiley and Sons, New York, 1983.

[7] W. Cantrell and A. Heymsfield. Production of ice in tropospheric clouds - a review. Bulletin of the American Meteorological Society, 86(6):795-807, 2005. 
[8] J. Carvalho and K. Dalnoki-Veress. Surface nucleation in the crystallisation of polyethylene droplets. The European Physical Journal E: Soft Matter and Biological Physics, 34(1):1-6, 2011.

[9] J. L. Carvalho and K. Dalnoki-Veress. Homogeneous bulk, surface, and edge nucleation in crystalline nanodroplets. Physical Review Letters, 105(23):237801, 2010.

[10] B. K. Chakraverty and G. M. Pound. Heterogeneous nucleation at macroscopic steps. Acta Metallurgica, 12(8):851-860, 1964.

[11] Y. Chushak and L. S. Bartell. Crystal nucleation and growth in large clusters of sef6from molecular dynamics simulations. The Journal of Physical Chemistry A, 104(41):9328-9336, 2000.

[12] V. G. Ciobanu, C. Marcolli, U. K. Krieger, A. Zuend, and T. Peter. Efflorescence of ammonium sulfate and coated ammonium sulfate particles: Evidence for surface nucleation. Journal of Physical Chemistry A, 114(35):9486-9495, 2010.

[13] W. A. Cooper. A possible mechanism for contact nucleation. Journal of the Atmospheric Sciences, 31(7):1832-1837, 1974.

[14] P.-G. de Gennes, F. Brochard Wyart, and D. Quéré. Capillarity and Wetting Phenomena. Springer, 2004.

[15] Y. Diao, T. Harada, A. S. Myerson, T. A. Hatton, and B. L. Trout. The role of nanopore shape in surface-induced crystallization. Nature Materials, 10(11):867-871, 2011.

[16] Y. S. Djikaev and E. Ruckenstein. Thermodynamics of heterogeneous crystal nucleation in contact and immersion modes. The Journal of Physical Chemistry. A, 112(46):11677-87, 2008. 
[17] Y. S. Djikaev, A. Tabazadeh, P. Hamill, and H. Reiss. Thermodynamic conditions for the surface-stimulated crystallization of atmospheric droplets. The Journal of Physical Chemistry A, 106(43):10247-10253, 2002.

[18] D. Duft and T. Leisner. Laboratory evidence for volume-dominated nucleation of ice in supercooled water microdroplets. Atmospheric Chemistry and Physics, 4(7):1997-2000, 2004.

[19] A. J. Durant and R. A. Shaw. Evaporation freezing by contact nucleation inside-out. Geophysical Research Letters, 32(20), 2005.

[20] B. Ervens and G. Feingold. Sensitivities of immersion freezing: Reconciling classical nucleation theory and deterministic expressions. Geophysical Research Letters, 40(12):3320-3324, 2013.

[21] L. F. Evans and J. E. Lane. Line tension and ice nucleation theory. Journal of the Atmospheric Sciences, 30(2):326-331, 1973.

[22] H. Eyring. The activated complex in chemical reactions. The Journal of Chemical Physics, 3(2):107, 1935.

[23] A. P. Fornea, S. D. Brooks, J. B. Dooley, and A. Saha. Heterogeneous freezing of ice on atmospheric aerosols containing ash, soot, and soil. Journal of Geophysical Research-Atmospheres, 114, 2009.

[24] S. K. Ghandhi. VLSI Fabrication Principles Silicon and Gallium Arsenide. Wiley, 2nd edition, 2011.

[25] J. W. Gibbs. On the equilibrium of heterogeneous substances. Transactions of the Connecticut Academy of Arts and Sciences, 111, 1874. 
[26] M. P. Goertz, X. Y. Zhu, and J. E. Houston. Exploring the liquid-like layer on the ice surface. Langmuir, 25(12):6905-6908, 2009.

[27] R. Goody. Principles of Atmospheric Physics and Chemistry. Oxford University Press, 1995.

[28] R. D. Gretz. Linetension effect in a surface energy model of a capshaped condensed phase. The Journal of Chemical Physics, 45(3160):3160-3161, 1966.

[29] R. Grossier, Z. Hammadi, R. Morin, and S. Veesler. Predictive nucleation of crystals in small volumes and its consequences. Physical Review Letters, 107(2), 2011.

[30] C. Gurganus, J. Charnawskas, A. Kostinski, and R. Shaw. Nucleation at the contact line observed on nanotextured surfaces. Physical Review Letters, 113(23):235701, 2014.

[31] C. Gurganus, A. B. Kostinski, and R. A. Shaw. Fast imaging of freezing drops: No preference for nucleation at the contact line. Journal of Physical Chemistry Letters, 2(12):1449-1454, 2011.

[32] C. Gurganus, A. B. Kostinski, and R. A. Shaw. High-speed imaging of freezing drops: Still no preference for the contact line. The Journal of Physical Chemistry C, 117(12):6195-6200, 2013.

[33] E. Hecht. Optics. Addison Wesley, San Fransisco, CA, 4th edition, 2002.

[34] R. Hickling. Nucleation of freezing by cavity collapse and its relation to cavitation damage. Nature, 206(4987):915-917, 1965.

[35] A. I. Hienola, P. M. Winkler, P. E. Wagner, H. VehkamÃd'ki, A. Lauri, I. Napari, and M. Kulmala. Estimation of line tension and contact angle from heterogeneous nucleation experimental data. The Journal of Chemical Physics, 126(9), 2007. 
[36] J. P. Hindmarsh, A. B. Russell, and X. D. Chen. Observation of the surface and volume nucleation phenomena in undercooled sucrose solution droplets. Journal of Physical Chemistry C, 111(16):5977-5981, 2007.

[37] J. Hirth and G. Pound. Progress in Materials Science: Condensation and Evaporation. Pergamon Press, 1963.

[38] J. L. Holbrough, J. M. Campbell, F. C. Meldrum, and H. K. Christenson. Topographical control of crystal nucleation. Crystal Growth \& Design, 12(2):750-755, 2012.

[39] C. Hoose and O. Mohler. Heterogeneous ice nucleation on atmospheric aerosols: a review of results from laboratory experiments. Atmospheric Chemistry and Physics, 12(20):9817-9854, 2012.

[40] S. Jung, M. Dorrestijn, D. Raps, A. Das, C. M. Megaridis, and D. Poulikakos. Are superhydrophobic surfaces best for icephobicity? Langmuir, 2011.

[41] S. Jung, M. K. Tiwari, N. V. Doan, and D. Poulikakos. Mechanism of supercooled droplet freezing on surfaces. Nature Communications, 3:615, 2012.

[42] D. A. Knopf. Do nad and nat form in liquid stratospheric aerosols by pseudoheterogeneous nucleation? The Journal of Physical Chemistry A, 110(17):5745-5750, 2006.

[43] A. Kostinski and W. Cantrell. Entropic aspects of supercooled droplet freezing. Journal of the Atmospheric Sciences, 65(9):2961-2971, 2008.

[44] D. Lamb and J. Verlinde. Physics and Chemistry of Clouds. Cambridge University Press, 2011. 
[45] T. Li, D. Donadio, L. M. Ghiringhelli, and G. Galli. Surface-induced crystallization in supercooled tetrahedral liquids. Nature Materials, 8(9):726-730, 2009.

[46] C. Lobban, J. Finney, and W. Kuhs. The structure of a new phase of ice. Nature, 391(6664):268-270, 1998.

[47] U. Lohmann and J. Feichter. Global indirect aerosol effects: a review. Atmospheric Chemistry and Physics, 5(3):715-737, 2005.

[48] Y. Lü and M. Chen. Surface layering-induced crystallization of ni-si alloy drops. Acta Materialia, 60(11):4636-4645, 2012.

[49] A. Marmur. Line tension and the intrinsic contact angle in solid-liquid-fluid systems. Journal of Colloid and Interface Science, 186(2):462-6, 1997.

[50] A. Marmur. Line tension effect on contact angles: Axisymmetric and cylindrical systems with rough or heterogeneous solid surfaces. Colloids and Surfaces A: Physicochemical and Engineering Aspects, 136(1-2):81-88, 1998.

[51] E. Mendez-Villuendas and R. Bowles. Surface nucleation in the freezing of gold nanoparticles. Physical Review Letters, 98(18), 2007.

[52] B. Murray, S. Broadley, T. Wilson, S. Bull, R. Wills, H. Christenson, and E. Murray. Kinetics of the homogeneous freezing of water. Physical Chemistry Chemical Physics, 12(35):10380-10387, 2010.

[53] B. Murray, D. O'Sullivan, J. Atkinson, and M. Webb. Ice nucleation by particles immersed in supercooled cloud droplets. Chemical Society Reviews, 41(19):6519-6554, 2012.

[54] G. Navascues and P. Tarazona. Line tension effects in heterogeneous nucleation theory. The Journal of Chemical Physics, 75(5):2441, 1981. 
[55] D. Niedermeier, S. Hartmann, R. Shaw, D. Covert, T. Mentel, J. Schneider, L. Poulain, P. Reitz, C. Spindler, T. Clauss, et al. Heterogeneous freezing of droplets with immersed mineral dust particles-measurements and parameterization. Atmospheric Chemistry and Physics, 10(8):3601-3614, 2010.

[56] J. Niehaus, K. W. Bunker, S. China, A. Kostinski, C. Mazzoleni, and W. Cantrell. A technique to measure ice nuclei in the contact mode. Journal of Atmospheric and Oceanic Technology, 31(4):913-922, 2014.

[57] M. Ovtchinnikov and Y. L. Kogan. An investigation of ice production mechanisms in small cumuliform clouds using a $3 \mathrm{~d}$ model with explicit microphysics. part i: Model description. Journal of the Atmospheric Sciences, 57(18):2989-3003, 2000.

[58] A. J. Page and R. P. Sear. Heterogeneous nucleation in and out of pores. Physical Review Letters, 97(6):065701, 2006.

[59] V. T. J. Philips, L. J. Donner, and S. T. Garner. Nucleation processes in deep convection simulated by a cloud-system-resolving model with double-moment bulk microphysics. Journal of the Atmospheric Sciences, 64(3):738-761, 2007.

[60] T. Pompe and S. Herminghaus. Three-phase contact line energetics from nanoscale liquid surface topographies. Physical Review Letters, 85(9):1930, 2000.

[61] H. R. Pruppacher and J. D. Klett. Microphysics of Clouds and Precipitation. Kluwer Academic Publishers, Boston, MA, 1997.

[62] J. S. Rowlinson and B. Widom. Molecular Theory of Capillarity, volume sec. 8.6. Dover, Oxford, U.K., 1982.

[63] S. Sastry. Water: Ins and outs of ice nucleation. Nature, 438:746-747, 2005. 
[64] R. P. Sear. Nucleation at contact lines where fluid-fluid interfaces meet solid surfaces. Journal of Physics-Condensed Matter, 19(46), 2007.

[65] R. P. Sear. Crystal nucleation: In a tight corner. Nature Materials, 10(11):809-810, 2011.

[66] R. P. Sear. The non-classical nucleation of crystals: microscopic mechanisms and applications to molecular crystals, ice and calcium carbonate. International Materials Reviews, 57(6):328-356, 2012.

[67] R. P. Sear. Generalisation of levine's prediction for the distribution of freezing temperatures of droplets: a general singular model for ice nucleation. Atmospheric Chemistry and Physics, 13(14):7215-7223, 2013.

[68] R. A. Shaw, A. J. Durant, and Y. Mi. Heterogeneous surface crystallization observed in undercooled water. The Journal of Physical Chemistry B, 109(20):9865-9868, 2005.

[69] O. G. Shpyrko, R. Streitel, V. S. Balagurusamy, A. Y. Grigoriev, M. Deutsch, B. M. Ocko, M. Meron, B. Lin, and P. S. Pershan. Surface crystallization in a liquid au-si alloy. Science, 313(5783):77-80, 2006.

[70] Ó. F. Sigurbjörnsson and R. Signorell. Volume versus surface nucleation in freezing aerosols. Physical Review E, 77(5):051601, 2008.

[71] R. Stockle, C. Fokas, V. Deckert, R. Zenobi, B. Sick, B. Hecht, and U. P. Wild. High-quality near-field optical probes by tube etching. Applied Physics Letters, 75(2):160, 1999.

[72] P. W. Sutter and E. A. Sutter. Dispensing and surface-induced crystallization of zeptolitre liquid metal-alloy drops. Nature Materials, 6(5):363-366, 2007. 
[73] S. Suzuki, A. Nakajima, N. Yoshida, M. Sakai, A. Hashimoto, Y. Kameshima, and K. Okada. Freezing of water droplets on silicon surfaces coated with various silanes. Chemical Physics Letters, 445(1-3):37-41, 2007.

[74] A. Tabazadeh, Y. S. Djikaev, and H. Reiss. Surface crystallization of supercooled water in clouds. Proceedings of the National Academy of Sciences of the United States of America, 99(25):15873-8, 2002.

[75] B. V. Toshev, D. Platikanov, and A. Scheludko. Line tension in three-phase equilibrium systems. Langmuir, 4(3):489-499, 1988.

[76] G. W. Turner and L. S. Bartell. On the probability of nucleation at the surface of freezing drops. The Journal of Physical Chemistry A, 109(31):6877-6879, 2005.

[77] J. A. van Meel, R. P. Sear, and D. Frenkel. Design principles for broad-spectrum protein-crystal nucleants with nanoscale pits. Physical Review Letters, 105(20):205501, 2010.

[78] L. Vrbka and P. Jungwirth. Homogeneous freezing of water starts in the subsurface. The Journal of Physical Chemistry B, 110(37):18126-18129, 2006.

[79] J. H. Weijs, A. Marchand, B. Andreotti, D. Lohse, and J. H. Snoeijer. Origin of line tension for a lennard-jones nanodroplet. Physics of Fluids (1994-present), 23(2):022001, 2011.

[80] B. Widom. Line tension and the shape of a sessile drop. The Journal of Physical Chemistry, 99(9):2803-2806, 1995.

[81] R. Williams and A. M. Goodman. Wetting of thin layers of sio2 by water. Applied Physics Letters, 25(10):531-532, 1974. 